\title{
Probing Pulsar Emission on Short Timescales: Rotating Radio Transients, Cyclic Spectroscopy, and Single-Pulse Studies of Millisecond Pulsars
}

Nipuni Tharaka Palliyaguru

Follow this and additional works at: https://researchrepository.wvu.edu/etd

\author{
Recommended Citation \\ Palliyaguru, Nipuni Tharaka, "Probing Pulsar Emission on Short Timescales: Rotating Radio Transients, \\ Cyclic Spectroscopy, and Single-Pulse Studies of Millisecond Pulsars" (2015). Graduate Theses, \\ Dissertations, and Problem Reports. 6372. \\ https://researchrepository.wvu.edu/etd/6372
}

This Dissertation is protected by copyright and/or related rights. It has been brought to you by the The Research Repository @ WVU with permission from the rights-holder(s). You are free to use this Dissertation in any way that is permitted by the copyright and related rights legislation that applies to your use. For other uses you must obtain permission from the rights-holder(s) directly, unless additional rights are indicated by a Creative Commons license in the record and/ or on the work itself. This Dissertation has been accepted for inclusion in WVU Graduate Theses, Dissertations, and Problem Reports collection by an authorized administrator of The Research Repository @ WVU.

For more information, please contact researchrepository@mail.wvu.edu. 


\section{Probing Pulsar Emission on Short Timescales: Rotating Radio Transients, Cyclic Spectroscopy, and Single-Pulse Studies of Millisecond Pulsars}

\section{Nipuni Tharaka Palliyaguru}

Dissertation submitted to the

Eberly College of Arts and Sciences at West Virginia University

in partial fulfillment of the requirements

for the degree of

Doctor of Philosophy

in

Physics

Dr. Maura McLaughlin, Ph.D., Chair

Dr. Duncan Lorimer, Ph.D.

Dr. D. J. Pisano, Ph.D.

Dr. Alan Bristow, Ph.D.

Dr. Zachariah Etienne, Ph.D.

Department of Physics and Astronomy

Morgantown, West Virginia

2015

Keywords: Pulsars; Neutron Stars

Copyright 2015 Nipuni T. Palliyaguru 


\title{
ABSTRACT \\ Probing Pulsar Emission on Short Timescales: Rotating Radio Transients, Cyclic Spectroscopy, and Single-Pulse studies of Millisecond Pulsars
}

\author{
Nipuni T. Palliyaguru
}

Rotating radio transients (RRATs) are neutron stars are that characterized by the emission of strong sporadic bursts. We have analysed the long- and shortterm time dependence of the pulse arrival times and the pulse detection rates for eight RRAT sources from the Parkes Multi-beam Pulsar Survey (PMPS). We find significant periodicities in the individual pulse arrival times from six RRATs. These periodicities range from $\sim 30$ minutes to 2100 days and from one to 16 independent (i.e. non-harmonically related) periodicities are detected for each RRAT. In addition, we find that pulse emission is a random process on short (hour-long) time scales but that most of the objects exhibit longer term (months-years) non-random behaviour. We find that PSRs J1819-1458 and J1317-5759 emit more doublets (two consecutive pulses) and triplets (three consecutive pulses) than is expected in random pulse distributions. No evidence for such an excess is found for the other RRATs. There are several different models for RRAT emission depending on both extrinsic and intrinsic factors which are consistent with these properties.

Light travel time changes due to gravitational waves may be detected within the next decade through precision timing of an array of millisecond pulsars. Removal of frequency-dependent interstellar medium (ISM) delays due to dispersion and scattering is a key issue in the detection process. Current timing algorithms routinely correct pulse times of arrival (TOAs) for time-variable delays due to cold plasma dispersion. However, none of the major pulsar timing groups routinely correct for delays due to scattering from multi-path propagation in the ISM. Scattering introduces a phase change in the signal that results in pulse broadening and arrival time delays. As a step toward a more comprehensive ISM propagation delay correction, we demonstrate through a simulation that we can accurately recover pulse broadening functions (PBFs), such as those that would be introduced by multi-path scattering, with a realistic signal-to-noise ratio, with consequent improvements in timing precision. We also demonstrate that we can isolate the scattering delays from other types of delays, and show that reductions in the timing residual root-meansquare of more than a factor of two are possible through removal of time-variable scattering delays. We also show that the effect of pulse-to-pulse "jitter" is not a serious problem for PBF reconstruction, at least for jitter levels comparable to those observed in several bright pulsars. 


\section{Acknowledgments}

I would like to thank Drs. Nancy Giles and Earl Scime for accepting me into the Ph.D. program at West Virginia University. I also thank the professors whom I had all kinds of great classes with, especially Dr. Weldon, for his excellent E\&M classes, for clarifying the physics that I hadn't understood before.

I also thank Dr. Maura McLaughlin for supervising me on the research projects and giving me the opportunity to get involved with NANOGrav and IPTA. Working with her has made me understand the importance of attention to detail and presenting things perfectly, which are skills that I am still trying to master. Being part of NANOGrav gave me the opportunity to discuss work with and present to a friendly audience which definitely helped build confidence. I will miss attending the yearly IPTA meetings and meeting everyone at various exotic places on earth. I also thank Dan Stinebring for all his guidance, the time he spent explaining cyclic spectroscopy, ISM effects and pulsar timing through webex on weekly meetings. I also thank him for the hospitality on various visits to Oberlin. The experience I gained working with Paul Demorest, Glenn Jones, Stefan Oslowski, Delphine Perrodine and Sarah Burke-Spolaor is invaluable. They have helped in numerous ways when things wouldn't work out as expected. I felt very motivated whenever I talked to them and I like to think that their energy and enthusiasm rubbed on me a great deal. I also thank the faculty at WVU for letting me sit in their classes, for providing feedback on applications, helpful discussions, advice when making important decisions and helping out in times of need. Next, I thank the dissertation committee members, Dr. Duncan Lorimer, Dr. D.J. Pisano, Dr. Alan Bristow, and Dr. Zach Etienne for providing helpful comments about this work.

A big thank you to Nate, for fixing all the computer and software issues and helping to relieve the pain of dealing with dspsr, PSRCHIVE tempo2 and whatnot. I would also like to thank the administrative staff Sherry, Greg, Siobhan, Audrey, Lori and Megan for their great support on various occasions.

I am also grateful for my parents, sister and friends who have supported me throughout these years. Grad school would not have been fun if it wasn't for my fellow graduate students and postdocs Akshaya, Lina, Manjari, Jayanth and my current office mates Lucas and Pete. I thank them for being there and supporting me through ups and downs in grad school. I also thank my Sri Lankan friends in Morgantown for always helping out, cooking delicious Sri Lankan food and making the years far away from home pleasant and memorable. 


\section{Table of Contents}

List of Tables vi

List of Figures $\quad$ vii

List of Abbreviations and Symbols viii

1 Introduction 1

1.1 Why study pulsars ...................... 1

1.2 Pulsar emission mechanisms . . . . . . . . . . . . . . . . 2

1.2.1 The magnetosphere . . . . . . . . . . . . . . . . 4

1.2.2 Emission beam and pulse shapes . . . . . . . . . . . 8

1.3 Classes of pulsars . . . . . . . . . . . . . . . . . . . . 10

1.3.1 Rotating radio transients . . . . . . . . . . . . . . 11

1.3.2 Millisecond pulsars . . . . . . . . . . . . . . . . 13

1.4 Interstellar medium effects . . . . . . . . . . . . . . . . . . . . . 14

1.4.1 Dispersion ........................ . . 14

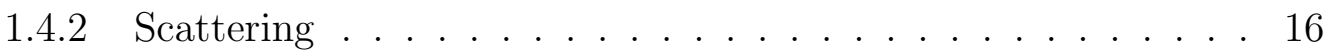

1.5 Pulsar data processing . . . . . . . . . . . . . . . . . . . . . . . . . . . . . . . . . . . . . .

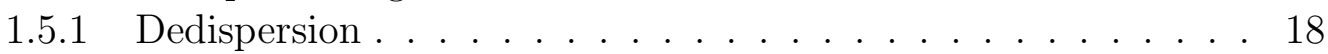

1.5.2 Obtaining single pulses and folded profiles . . . . . . . . . 18

1.5.3 Radio frequency interference removal . . . . . . . . . . . . . . 19

1.5.4 Flux calibration ................. . 20

1.5.5 Polarization calibration ................ 23

1.6 Pulsar timing . . . . . . . . . . . . . . . . . . . . 25

1.7 Using millisecond pulsars to detect gravitational waves . . . . . . . 28

2 Radio Properties of Rotating Radio Transients 31

2.1 Introduction . . . . . . . . . . . . . . . . . . 31

2.2 Observations . . . . . . . . . . . . . . . . . . . . . 34

2.3 Analysis and Results . . . . . . . . . . . . . . . . 36

2.3.1 Periodicity search ...................... 36

2.3.1.1 Periodicity in pulse arrival times . . . . . . . . . 41

2.3.1.2 Periodicities in daily pulse detection rates . . . . . 44

2.3.2 Randomness tests on pulse arrival times . . . . . . . . . . 46

2.4 Discussion . . . . . . . . . . . . . . . . . . . . . 51

3 Interstellar Time Delay Correction $\quad 57$

3.1 Introduction . . . . . . . . . . . . . . . . . . 57

3.2 Theoretical background . . . . . . . . . . . . . . . 62

3.3 Simulations . . . . . . . . . . . . . . . . . . 67

3.3.1 Scatter correction ................. . . . 70

3.3.2 Effects of pulse-to-pulse jitter . . . . . . . . . . . . . 80

3.3.3 Presence of non-scattering delays . . . . . . . . . . . . . . . 81 
3.4 Discussion . . . . . . . . . . . . . . . . . . . . . . . 84

4 Single pulse properties of J1713+0747 88

4.1 Introduction . . . . . . . . . . . . . . . . . . . 88

4.2 Observations and data processing . . . . . . . . . . . . . . . . . . . . . . . . . . . . . . . . 91

4.3 Data analysis and results . . . . . . . . . . . . . . . . . . . . . 92

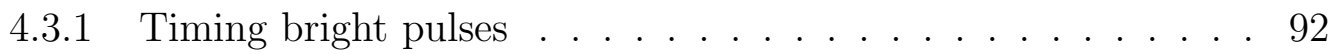

4.4 Discussion . . . . . . . . . . . . . . . . . . . . 96

5 Conclusions and future work 98

A Signal to Noise Ratio for CS Reconstruction 102 


\section{List of Tables}

2.1 Periodicities in the pulse detection rates . . . . . . . . . . . . 40

2.2 Periodicities in the arrival times . . . . . . . . . . . . . . . . 43

2.3 KS test results of randomness . . . . . . . . . . . . . . . . . . . . . . . . . . . .

2.4 Expected and measured multiplets of pulses . . . . . . . . . . . 51

4.1 Pulse widths, S/N and RMS residuals of J1713+0747 . . . . . . . 96 


\section{List of Figures}

1.1 Schematic view of a pulsar magnetosphere . . . . . . . . . . . 5

1.2 Beam geometry . . . . . . . . . . . . . . . . . . . 9

1.3 Core and conal beam structure and patchy beam . . . . . . . . . 10

$1.4 \quad P-\dot{P}$ diagram of pulsars . . . . . . . . . . . . . . . 12

1.5 Relationship between antenna temperature and flux density . . . . . 21

1.6 Example of Timing Residuals . . . . . . . . . . . . . . . 27

2.1 Periodicities in the TOAs . . . . . . . . . . . . . . . 38

2.2 Detection rate for eight RRATs . . . . . . . . . . . . . . . . . . . . . . . . . . . . . .

2.3 Periodicities in the detection rates . . . . . . . . . . . . . . . . . . . . . . . . . . 40

2.4 CDF of pulse sequences . . . . . . . . . . . . . . . . . . . 48

3.1 Toy model of delays . . . . . . . . . . . . . . . . . . . . . . . . . 62

3.2 Scatter-broadened profile . . . . . . . . . . . . . . . . . . . . . . . . . . . . 70

3.3 Cyclic spectrum . . . . . . . . . . . . . . . . . 71

3.4 PBF recovery . . . . . . . . . . . . . . . . . . . 72

3.5 TOA correction for scattering . . . . . . . . . . . . . . . 74

3.6 Scatter correction vs S/N . . . . . . . . . . . . . . 76

3.7 The $\chi^{2}$ of residuals as a function of average profile $\mathrm{S} / \mathrm{N} \ldots \ldots$. . . . 77

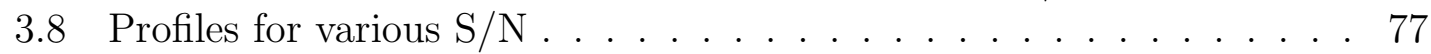

3.9 Scattering timescales from cyclic spectroscopy . . . . . . . . . . 78

3.10 Scatter correction vs mean scattering timescale . . . . . . . . . . . . 79

3.11 The $\chi^{2}$ of residuals as a function of the mean input scattering timescale 79

3.12 Jittered profiles and recovered PBFs . . . . . . . . . . . . . . 82

3.13 Scatter correction in the presence of a GW signal . . . . . . . . . 83

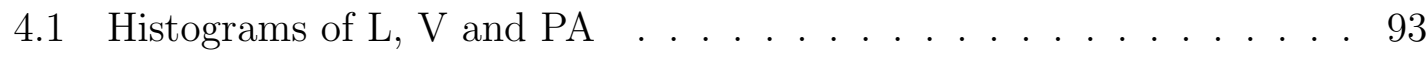

4.2 A bright single pulse and the average profile . . . . . . . . . . . . 94

4.3 RMS residuals vs $\mathrm{S} / \mathrm{N}$ and the number of pulses in the composite profile 95 


\section{Glossary, List of Abbreviations and Symbols}

System temperature: Temperature of the resistive load in the telescope that measures the power from sources

Telescope gain: A constant that relates the temperature and flux

Dispersion measure (DM): Integral of the electron density along the line of sight

Dynamic spectrum: Pulsar intensity as a function of radio frequency and time that shows maxima and minima of the interference pattern

Scintle: Maxima in the interference pattern seen in the dynamic spectrum

Cyclic spectrum: Pulsar intensity as a function of radio frequency and cycle frequency

Pulse-to-pulse jitter: Shape and phase changes of pulses on single pulse timescales Polarization position angle (PA): Angle between the rotation axis and the projected magnetic field line

$\begin{array}{ll}\text { NS } & \text { Neutron star } \\ \text { GR } & \text { General relativity } \\ \text { GW } & \text { Gravitational wave } \\ \text { MSP } & \text { Millisecond pulsar } \\ \text { DM } & \text { Dispersion measure } \\ \text { PA } & \text { Linear polarization position angle } \\ \text { TOA } & \text { Time of arrival } \\ \text { Fermi } & \text { Fermi Gamma-ray Telescope } \\ M_{\odot} & \text { Solar mass } \\ \mathrm{R}_{\mathrm{NS}} & \text { Neutron star radius }(=10 \mathrm{~km}) \\ c & \text { Speed of light } \\ m & \text { Magnetic moment }(=|\vec{m}|) \\ I & \text { Neutron star moment of inertial }\left(=10^{45} \mathrm{~g} \mathrm{~cm}^{2}\right) \\ \mathrm{R}_{\mathrm{LC}} & \text { Light cylinder radius } \\ D & \text { Distance to the pulsar } \\ \Omega & \text { Rotational angular frequency } \\ \mathrm{P} & \text { Spin period } \\ \dot{P} & \text { Derivative of the spin period } \\ n & \text { Braking index } \\ B_{\text {surf }} & \text { Surface magnetic field strength } \\ P_{0} & \text { Initial spin period } \\ \dot{P} & \text { Time derivative of the spin period } \\ \dot{E} & \text { Spin-down luminosity } \\ \mathrm{CS} & \text { Cyclic spectroscopy } \\ \Delta \nu_{D I S S} & \text { Scintillation bandwidth } \\ \Delta t_{D I S S} & \text { Scintillation timescale } \\ \mathrm{PBF} & \text { Pulse broadening function } \\ \mathrm{S} / \mathrm{N} & \text { Signal-to-noise ratio } \\ & \end{array}$




\section{Chapter 1}

\section{Introduction}

\subsection{Why study pulsars}

Pulsars are full of exciting physics. They are born as neutron stars leftover from supernova explosions. When stars with masses $>8 M_{\odot}$ evolve from the main sequence by burning $\mathrm{H}$ and He, forming heavier elements until an iron core is produced, they collapse under their own gravity until the star is supported by electron degeneracy pressure. Further collapse results in electrons and protons combining to form neutrons so that the star is supported by neutron degeneracy pressure. The outer layers that are not supported by neutron degeneracy pressure explode in a supernova releasing a large amount of energy that could be as great as $\sim 10^{43} \mathrm{ergs} \mathrm{s}^{-1}$ over a timescale of minutes to days. The core of the star is compressed to nuclear densities reaching up to $10^{15} \mathrm{~g} / \mathrm{cm}^{-3}$ by strong gravitational fields in the neutron star. This is a star with a mass of $1.4 \mathrm{M}_{\odot}-3 \mathrm{M}_{\odot}$ compressed to radius between 10 $15 \mathrm{~km}$ (Lattimer \& Prakash, 2007). Under these extreme conditions, matter could behave differently, that is the interiors of the star could be consist of quark-gluon plasma, in which the quarks and gluons that make up nucleons are deconfined. This kind of matter is expected to have been present at the very early stages of the universe and on Earth can only be re-produced in particle accelerators.

Neutron stars that emit a beam of radiation are known as pulsars and were 
first discovered through their periodic emission by Jocelyn Bell and Anthony Hewish (Hewish et al., 1968). Pulsars are characterized by their large magnetic fields $\sim 10^{12} \mathrm{G}$ and rapid rotation. While the magnetic fields from the original star, in which charged particles generate magnetic fields, strengthen with the core collapse, conservation of angular momentum before and after the supernova explosion leads to fast spins. See Gold (1968) and Pacini (1968) for the very first explanations of pulsating radio sources soon after the discovery. Due to the star's rotation with its magnetic field, there exists a region around it in which plasma co-rotates with the star. This region is called the magnetosphere. Two beams of radiation are emitted due to particle acceleration in the magnetosphere. The observed large brightness temperatures are attributed to a coherent emission mechanism (Ginzburg \& Zheleznyakov, 1970), where bunching of particles (Ruderman \& Sutherland, 1975) enhances the emission. Even after more than 40 years of discovery, the core composition, magnetosphere and the emission mechanism of these objects are yet to be fully understood. Therefore, pulsars can be used to test many aspects of fundamental physics, not only because they are perfect laboratories with high gravitational

fields, super-dense cores and highly magnetized environments but also because of their remarkable stability and timing accuracies.

\subsection{Pulsar emission mechanisms}

Pulsars are expected to be powered by the loss of rotational kinetic energy. As the star spins, we see pulses of electromagnetic radiation, due to a misalignment 
between the magnetic and rotational axes. If the magnetic misalignment is zero, from Equation 1.1, no energy will be lost and therefore no radio emission will be observed.From classical electrodynamics, the power radiated by a rotating magnetic dipole with a magnetic moment $\vec{m}$ (Jackson, 1962) is,

$$
\dot{E}_{\text {dipole }}=\frac{2}{3 c^{3}} m^{2} \Omega^{4} \sin ^{2} \alpha
$$

where $\alpha$ is the misalignment of the magnetic axis from the rotation axis and $c$ is the speed of light.

With the rotational energy $E_{\text {rot }}=I \Omega^{2} / 2$, we write the rotational kinetic energy loss as

$$
\dot{E}=\frac{d E_{\mathrm{rot}}}{d t}=\frac{d\left(I \Omega^{2} / 2\right)}{d t}=I \Omega \dot{\Omega}=4 \pi^{2} I \dot{P} P^{-3}
$$

where $\Omega$ is the rotational angular frequency, $P$ is the spin period, and $\dot{P}$ is the time derivative $\dot{P}=\frac{d P}{d t}$ of the spin period. This quantity $\dot{E}$ is known as the "spin-down luminosity" of the pulsar.

By equating the spin-down luminosity (Equation 1.2) with $\dot{E}_{\text {dipole }}$, we derive an expression for the rotational frequency evolution

$$
\dot{\Omega}=-\left(\frac{2 m^{2} \sin ^{2} \alpha}{3 I c^{3}}\right) \Omega^{3} .
$$

The magnetic moment is approximately equal to the magnetic field strength $B$ in the form of $B \approx m / r^{3}$, so that we get the surface magnetic field strength 


$$
B_{\text {surf }}=B(r=R)=\sqrt{\frac{3 c^{3} I}{8 \pi^{2} R^{6} \sin ^{2} \alpha} P \dot{P}} .
$$

For a typical NS with a mass of $1.4 M_{\odot}$, radius of $R_{\mathrm{NS}}=10 \mathrm{~km}$ and moment of inertia $I=10^{45} \mathrm{~g} \mathrm{~cm}^{2}$, we find a rough estimate for the inferred magnetic field as

$$
B_{\text {surf }}=3.2 \times 10^{19} \sqrt{P \dot{P}} \text { Gauss } \simeq 10^{12}\left(\frac{\dot{P}}{10^{-15}}\right)^{1 / 2}\left(\frac{P}{s}\right)^{1 / 2} \text { Gauss }
$$

for an orthogonal rotator (i.e. $\alpha=90^{\circ}$ ). From their measured periods and period derivatives, the inferred surface magnetic fields of observed pulsars are $\sim 10^{7}-10^{14} \mathrm{G}$ using Equation 1.5. Polarization properties can be used to measure $\alpha$ (Mitra \& Rankin, 2011) and have been found to have a large range with a peak at $35^{\circ}$ (Rankin, 1990).

\subsubsection{The magnetosphere}

Pulsar magnetospheres consist of relativistic pair plasmas formed by charged particles being pulled off from the stellar surface by strong electric fields. Figure 1.1 shows a toy model of a pulsar magnetosphere. According to Goldreich \& Julian (1969), the NS has a dipolar magnetic field and both the interior and exterior of the star are excellent electrical conductors. Therefore, with rotation, the star will be polarized and the surface charge density is quadrupolar, so that the outside electric field is a quadrupole (see Michel, 1991). In this simplest model, considering the star to be a conducting sphere rotating with magnetic field $B$, the induced electric field 


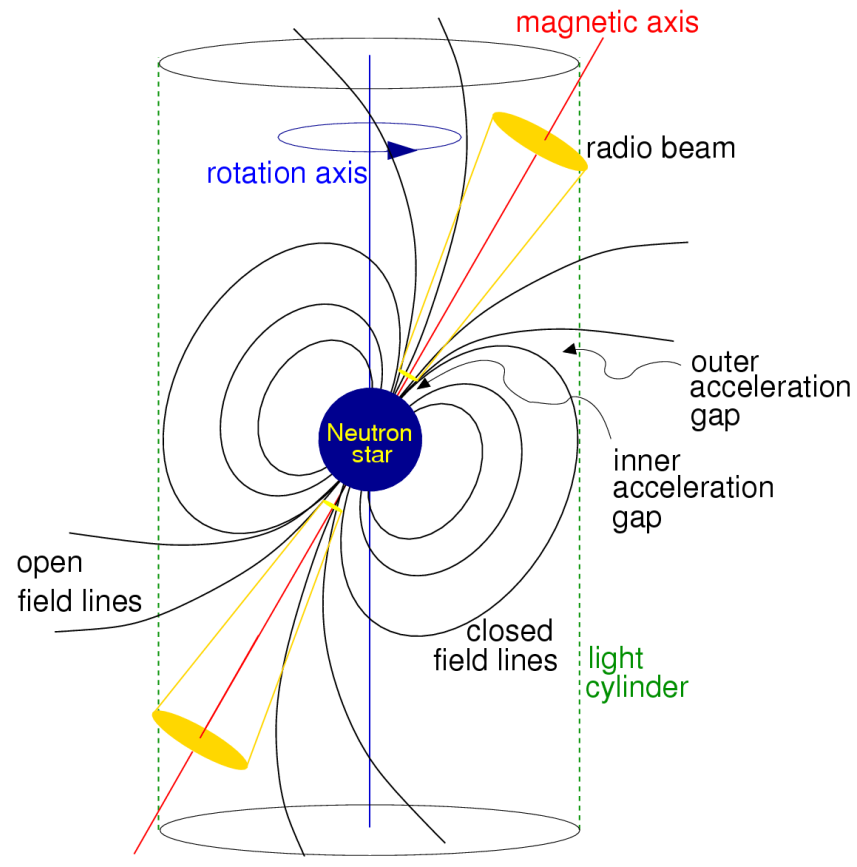

Figure 1.1: Lighthouse model of a rotating pulsar and its magnetosphere (Lorimer \& Kramer, 2005). The plasma filled region near the magnetic field lines is known as the magnetosphere. The magnetic axis is misaligned with the vertical rotation axis. The co-rotating particles obtain the speed of light at the light cylinder radius shown by dotted lines. The field lines that close within the light cylinder are known as closed field lines. The field lines which are outside of this boundary are known as open field lines. The radio beams are located at each pole of the NS centered around the magnetic axis. Particles are accelerated in the inner and outer acceleration gaps, which are regions depleted of plasma near the polar cap and in the outer magnetosphere. 
$E$ at a distance $r$, is given by

$$
\vec{E}=\frac{-1}{c}(\Omega \times r) \times \vec{B},
$$

The electric field potential of an external quadrupole field induced by surface charge densities is

$$
\Phi(r, \theta)=\frac{\Omega B_{s} R^{5}}{6 c r^{3}}\left(3 \cos ^{3} \theta-1\right),
$$

In polar coordinates $(r, \theta)$ of a star-centered coordinate system, the electric field component which is parallel to the magnetic field line on the NS surface is

$$
E_{\|}(r=R)=\left.\frac{\vec{E} \cdot \vec{B}}{B}\right|_{r=R}=-\frac{\Omega B_{0} R}{c} \cos ^{3} \theta
$$

where $B_{0}$ is the polar magnetic field at the surface, $R$ is the NS radius, $c$ is the speed of light, and $\theta$ is the polar angle. The outward electric force $\left(F=q E_{\|}\right)$exerted on the charged particles is orders of magnitudes greater than the gravitational force acting on the charged particles. Therefore, particles are pulled off from the surface of the star to populate the magnetosphere. The density of charged particles is given by

$$
\rho_{e}(r, \theta)=\frac{\vec{\nabla} \cdot \vec{E}}{4 \pi}=-\frac{\vec{\Omega} \cdot \vec{B}}{2 \pi c}=-\frac{B_{0} \Omega R^{3}}{4 \pi c r^{3}}\left(3 \cos ^{3} \theta-1\right) .
$$

This is known as the Goldreich-Julian density. According to this equation, there will be a region, at $\cos \theta=\sqrt{1 / 3}$ where the charge distribution changes sign.

These particles co-rotate with the star, up to a radius at which they reach the 
speed of light. This is called the light cylinder radius given by $R_{L C}=c / \Omega$. The magnetic field lines that close within this radius are called the closed field lines and the ones that do not close within this radius are called open field lines.

Many models predict the existence of gaps which are regions depleted of plasma. These gaps are necessary for emission because the presence of charges may screen the existing electric fields and thereby hinder particle acceleration. Inside the gap $\vec{E} \cdot \vec{B} \neq 0$ and a potential difference exists within the gap, whereas in the other regions of the magnetosphere where charged particles exist, $\vec{E} \cdot \vec{B}=0$ and the potential difference along a magnetic field line is almost negligible. Therefore, charged particles from the surface of the star are accelerated along magnetic field lines because of the existence of the electric field parallel to the field lines. The polar cap region, which is defined by the open field lines and the outer gap region, which is between the last closed field line and the first open field line, are two such regions that may generate radio and high-energy emission.

Radio emission is attributed to pair plasma $\left(e^{+}-e^{-}\right)$flowing outward along open magnetic field lines from the polar caps. The mechanisms responsible for this could either be curvature radiation or inverse Compton. Charged particles removed from the surface and traveling along the field lines produce two $\gamma$-ray photons. These charged particles are known as "primary particles". The $\gamma$ rays split due to the strong magnetic fields, producing an electron-positron pair, initiating a pair cascade. The newly generated pairs or the "secondary particles" and have low energies compared to those of primary particles and are responsible for radio emission. High-energy emission is likely to occur from the outer gap regions. This is 
further backed up by the high-energy profiles that are wider than the radio profiles.

Some pulsars show rotational irregularities that appear in the form of glitches and spin noise. These are generally identified as inconsistencies between the theoretically expected and the actual second derivative of the pulsar spin frequency $(\ddot{\nu})$. From an emission mechanism point-of-view, they are expected to arise from superfluid-crust interactions inside the star (Jones, 1990). From Equation 1.3, the rotational frequency evolution $\dot{\nu}$ can be expressed as

$$
\dot{\nu}=-K \nu^{n}
$$

where $n$ is the braking index and $n=3$ for magnetic dipole radiation. From this we expect, $\ddot{\nu}=n \dot{\nu}^{2} / \nu$.

Equation 1.10 can be written in terms of the period $P$ as $P=1 / \nu$ and integrated to obtain the age of the pulsar as

$$
T=\frac{P}{(n-1) \dot{P}}\left[1-\left(\frac{P_{0}}{P}\right)^{n-1}\right],
$$

where $P_{0}$ is the birth period. Assuming magnetic dipole radiation (i.e. $n=3$ ) and that $P_{0} \ll P$, the characteristic age can roughly be expressed as $\tau_{c}=P / 2 \dot{P}$.

\subsubsection{Emission beam and pulse shapes}

As described earlier, accelerated particles traveling along the open magnetic field lines emit photons tangential to the field lines. The angle between the field line 
(a)

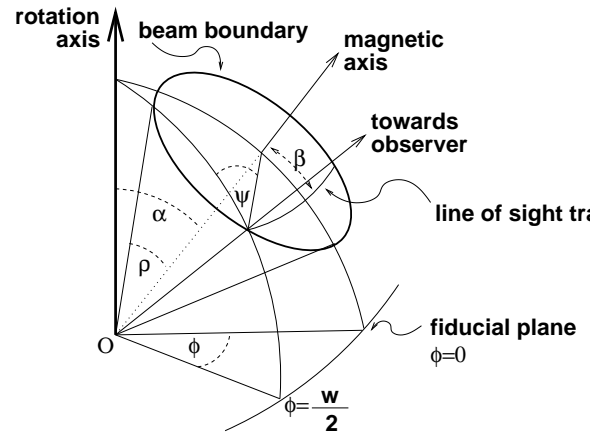

(b)

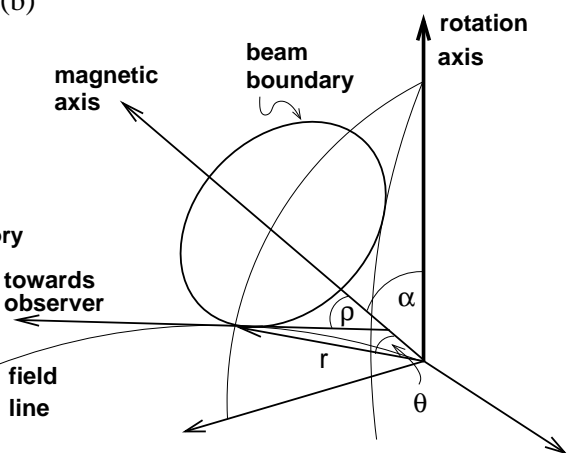

Figure 1.2: The magnetic axis is inclined to the rotation axis by and angle $\alpha$ and the emission cone has an opening angle $\rho$. The position angle $\Psi$ is measured with respect to the projected direction of the magnetic field lines and the pulse longitude at a particular point on the line of sight trajectory. $\beta$ is defined as the impact parameter or the closest approach point which occurs at longitude zero (Lorimer \& Kramer, 2005).

and the emitted photons is $\sim 1 / \gamma$, where $\gamma$ is the Lorentz factor. For large $\gamma$ values, the photons will be almost tangential to the field line. The emission geometry can be pictured as a cone with an opening angle $\rho$ to the magnetic axis, which also defines the open field line region as shown in Figure 1.2. The position angle $\Psi$, defined as the angle between the projected direction of the magnetic axis and the longitude lines at a given point on the line-of-sight trajectory. As the radiation beam sweeps past the line-of-sight, a pulse is seen and also $\Psi$ changes from $-\pi / 2$ to $+\pi / 2$ in an S-shaped curve (Radhakrishnan \& Cooke, 1969). However, many pulsars exhibit complicated profile shapes, which are explained by invoking a beam that is composed of a core and several cone structures. (Rankin, 1983a,b, 1990, 1993) Alternatively, they may also be explained by invoking the idea that the beam is randomly filled with emitting regions (Lyne \& Manchester, 1988) as shown in Figure 1.3. The observed pulse shapes depend on the section of the beam traversed 


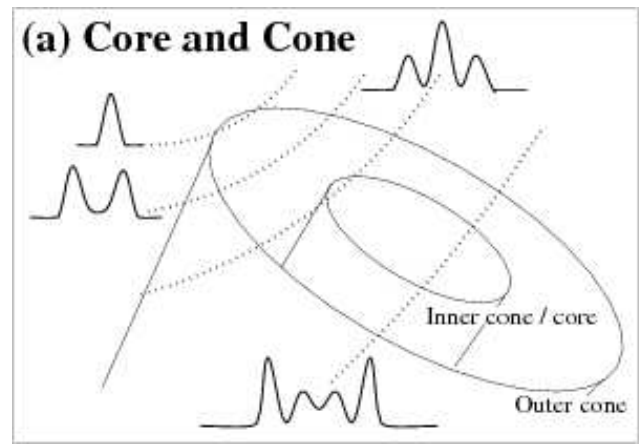

\section{(b) Patchy Beams}

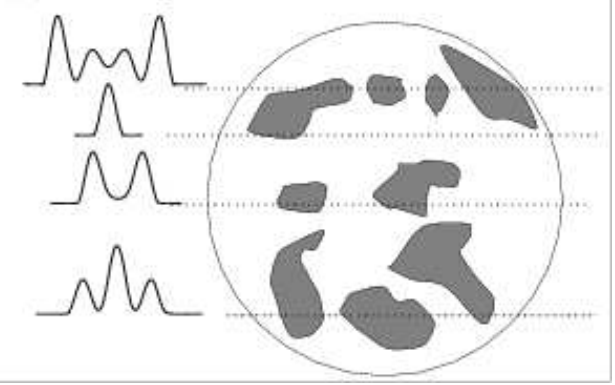

Figure 1.3: Schematic views of different beam structures (Lorimer \& Kramer, 2005). Dotted lines are different lines of sight across the beam and resultant pulse profiles are shown for (a) core and conal beam structure (b) patchy beam structure.

by the line-of-sight as the pulsar rotates, as shown in Figure 1.3.

Even though average profiles are quite stable, individual pulses are known to vary in pulse amplitude and phase. Random fluctuations in arrival phase can be of the order of a pulse width, a phenomenon known as pulse-to-pulse jitter (Cordes \& Shannon, 2010). The origin of this could be the incomplete filling of the openfield line region in the magnetosphere or altitude variations in the radio emission (Ruderman \& Sutherland, 1975). Furthermore, there can be shape changes on timescales of years (Lyne et al., 2009).

\subsection{Classes of pulsars}

Pulsars can be classified according to their period $P$ and period derivative $\dot{P}$. This is called a $P-\dot{P}$ diagram of pulsars as shown in Figure 1.4. Since inferred magnetic fields can be written in terms of $P$ and $\dot{P}$ as $B \propto \sqrt{P \dot{P}}$, the constant magnetic field strengths are depicted as negative gradient straight lines in this diagram.

Similarly, since the characteristic ages of pulsars are given by $\tau=P / 2 \dot{P}$, constant 
pulsar ages are given by the positive gradient straight lines. Three separate classes of objects can be identified in the diagram. These are the normal pulsar population, millisecond pulsars and magnetars. While normal pulsars have spin periods $\sim 1 \mathrm{~s}$

and period derivatives of $\sim 10^{-15} \mathrm{~s} / \mathrm{s}$, millisecond pulsars on the other hand rotate faster with spin periods $\sim 1.5-30 \mathrm{~ms}$, and smaller period derivatives of $\sim 10^{-19}$ s/s. Magnetars are characterized by their large magnetic fields, long periods and high energy bursts expected to be powered by the decay of magnetic fields (Duncan \& Thompson, 1994). While most magnetars are detected only in the $\gamma$ - and X-ray wavelengths, there have been recent discoveries of radio pulses from some objects (Camilo et al., 2006; Rea et al., 2013). A class of high-B field pulsars, that fall between the magnetars and radio pulsars, which have high magnetic fields but emission properties similar to normal pulsars have also been identified. In addition to these, there is a class of pulsars identified as rotating radio transients (RRATs), which are in the normal pulsar region but extend up to the magnetar region. The following sections discuss MSPs and RRATs in detail.

\subsubsection{Rotating radio transients}

Pulsars are generally searched for as periodic signals in time series data via a Fourier transform. However, some pulsars emit intermittently so that their emission may not be detected as periodic. In this situation a single-pulse-search needs to be carried out. Rotating radio transients (RRATs) are neutron stars which were discovered only through their isolated pulses (McLaughlin et al., 2009). The periods 


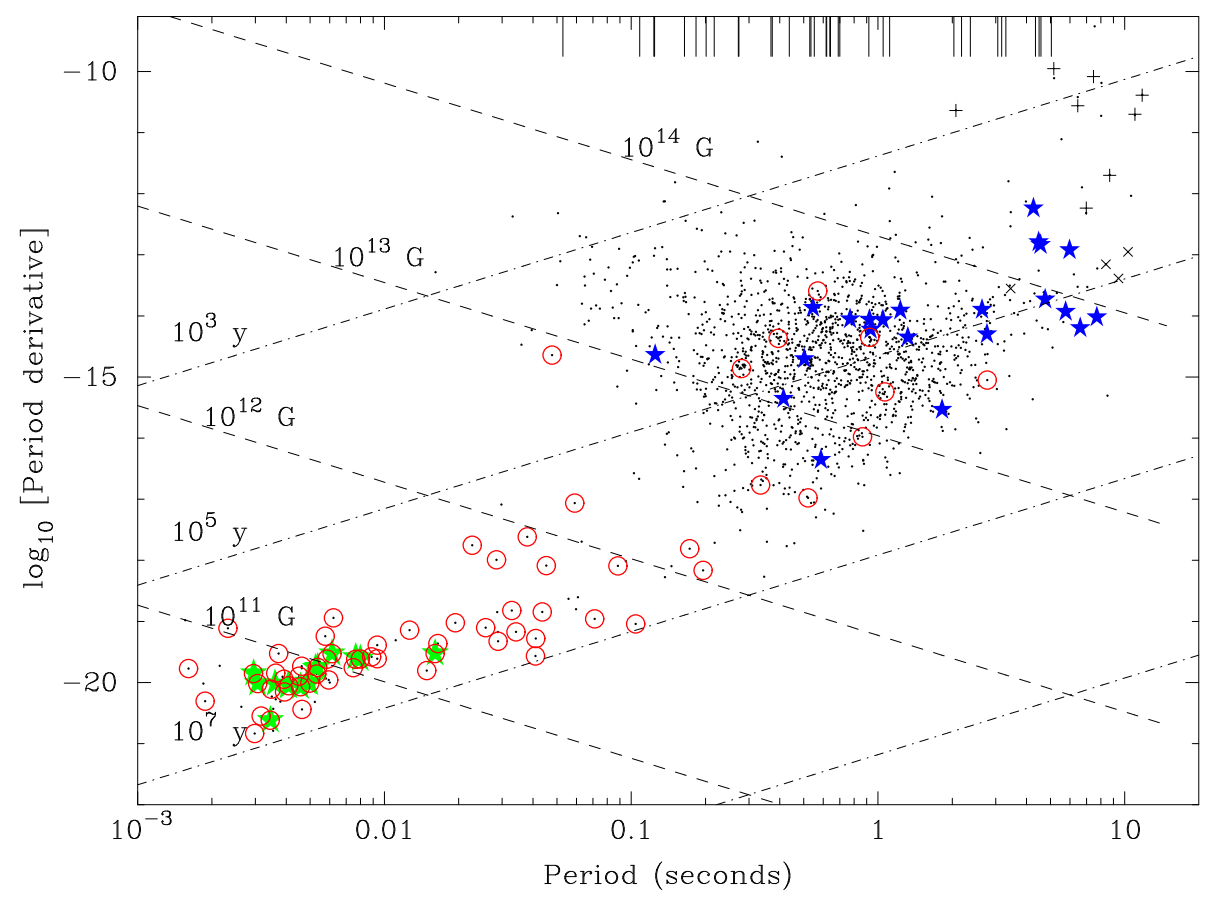

Figure 1.4: $P-\dot{P}$ diagram for known pulsars. Lines of constant spin-down luminosity, magnetic field and the characteristic age are marked by the dashed lines. Dots and red circle-dots represent isolated pulsars and binary pulsars, respectively. Plus signs represent soft gamma-ray repeaters (SGR) and anomalous X-ray pulsars (AXP) and crosses represent radio quiet X-ray isolated neutron stars (XINS). Green stars represent the MSPs timed by NANOGrav and the blue stars represent the RRATs. 
and magnetic fields of RRATs are generally larger than those of normal pulsars, but the distributions of other spin-down properties such as spin-down energy loss rate and characteristic age are similar. The sporadicity may be attributed to these objects being near the radio 'death-line' (e.g. Chen \& Ruderman, 1993; Zhang et al., 2007) and/or are examples of extreme nulling pulsars, which are pulsars that are mostly in the on-state and go into off states that may last for seconds to minutes, or as objects at the extreme end of the population of normal radio pulsars. A total of 90 RRATs $^{1}$ (Hessels et al., 2008; Burke-Spolaor \& Bailes, 2010a; Deneva et al., 2009; Keane et al., 2010, 2011) have been detected thus far. RRATs are discussed in more detail in Chapter 2.

\subsubsection{Millisecond pulsars}

These objects were first identified with the discovery of B1937+21 (Backer et al., 1982). They typically have magnetic fields of $\sim 10^{8} \mathrm{G}$, which are smaller than those of normal pulsars, and ages of $\sim 10^{9} \mathrm{yrs}$ which imply that they are older than normal pulsars. About $80 \%$ of the MSPs are in binary systems which leads to an explanation of their fast spin rates. When a neutron star is formed in a binary system, in which the companion evolves into a giant that fills its Roche lobe, the neutron star can accrete mass and angular momentum from the companion and spin-up. The isolated MSPs which are not in a binary are thought to be due to the star ablating the companion by relativistic particle winds (Ruderman et al., 1989). If the companion is massive enough to explode in a supernova, then a second

\footnotetext{
${ }^{1}$ http://astro.phys.wvu.edu/rratalog/
} 
neutron star is born, making the system a double neutron star binary. However, the common scenario is for the MSPs to be in a binary with a low mass white dwarf companion (Bhattacharya \& van den Heuvel, 1991). The MSPs detected so far in the Galaxy appear to be distributed isotropically but they could be more abundant in globular clusters and in the Galactic plane, since they consist of older stars and high stellar density environments. Many objects have been found through targeted searches performed on globular clusters and Fermi gamma ray sources as MSPs emit a larger proportion of their energy in gamma rays (Ray et al., 2013; Keith et al., 2011; Manchester et al., 1991).

\subsection{Interstellar medium effects}

The interstellar medium (ISM) consists of dust and gas, which is largely ionized plasma. As pulsar signals propagate through the ISM, it introduces frequency dependent delays due to dispersion and scattering. Scattering introduces a phase change in the signal that results in pulse broadening and arrival time delays. Additionally it affects the pulsar flux through scintillation and refractive effects. The following sections describe the basics of dispersion and scattering and Chapter 3 discusses their effect on pulsar timing in more detail.

\subsubsection{Dispersion}

The refractive index of this cold ionized plasma is frequency dependent, which means that the velocity of the propagating signal will depend on its wavelength. 
The refractive index can be expressed as

$$
\mu=\sqrt{1-\left(\frac{f_{p}}{f}\right)^{2}}
$$

where $f$ is frequency of the observed radiation and $f_{p}$ is the plasma frequency given by $f_{p}=\sqrt{e^{2} n_{e} / \pi m_{e}}$, for electron number density $n_{e}$, charge $e$ and mass $m_{e}$ of an electron.

Since the group velocity of a wave is $v_{g}=c \mu$, the high frequency waves experience a higher refractive index and thereby travel faster than the low frequency waves. When compared to a signal traveling in a vacuum, the delay introduced to the signal due to the cold plasma in the ISM is,

$$
t=\left(\int_{0}^{d} \frac{d l}{v_{g}}\right)-\frac{d}{c}
$$

Substituting for $v_{g}$ gives

$$
t=\frac{e^{2}}{2 \pi m_{e} c} \frac{\int_{0}^{d} n_{e} d l}{f^{2}}=D \times \frac{\mathrm{DM}}{f^{2}}
$$

where $D$ is a constant. The integral of the electron density along the line of sight is referred to as the dispersion measure (DM).

The delay between two frequencies is therefore given by

$$
\Delta t=4.15 \times 10^{6} \mathrm{~ms} \times\left(f_{1}^{-2}-f_{2}^{-2}\right) \times \mathrm{DM}
$$


Data are corrected for dispersion by calculating $\Delta t$ for each frequency and by shifting by that amount so that the arrival time of the pulse at both frequencies will be the same. This is discussed in detail in Section 1.5.1. When the DM is not known in the case of new pulsars, it is found by dedispersing at a range of trial DMs and finding the best value.

\subsubsection{Scattering}

A second prominent effect that is observed in pulsar signals is scattering due to to the inhomogeneities in the ISM. Radio waves usually do not undergo scattering from dust particles as they are of size $\sim 100 \mathrm{~nm}$, and the wavelength of the radio waves is much larges than the dust particles. However, radio waves propagating through the ISM undergo scattering from the free electrons in the plasma. As ion mass is larger than that of electrons, the acceleration of ions is negligible, therefore scattering due to ions can be ignored.

The most prominent effect of scattering on pulsar signals is pulse broadening which results from a phase change in the signal due to multipath propagation, where the signal travels through more than one path due to inhomogeneities in the medium. The ISM acts as a linear filter with a voltage pulse broadening function $(\mathrm{PBF})$, which is convolved with the intrinsic pulsar signal to produce the observed pulse. Therefore, scattering can cause delays in the time of arrival of pulses and may have serious implications for PTA efforts. There are several correction algorithms that are used to estimate scattering delays in pulsar data. Some of these meth- 
ods involve calculating the auto correlation function $(\mathrm{ACF})$ of the pulsar dynamic spectra, or the cumulative delay function from pulsar secondary spectra (see, e.g., Hemberger \& Stinebring, 2008). The dynamic spectrum gives the pulsar intensity as a function of frequency and time. The scintillation bandwidth and timescale are calculated by fitting a two-dimensional Gaussian to the ACF. The scattering timescale is calculated as $\tau \approx 1 / \Delta \nu_{D I S S}$, where $\Delta \nu_{D I S S}$ is the scintillation bandwidth, or the half width of the fitted Gaussian along the frequency axis. In addition to using the information in dynamic and secondary spectra, there algorithms which deconvolve the PBF from the observed signal. Cyclic spectroscopy (Demorest, 2011) is one such technique that we have used to recover PBFs from simulated pulse profiles, and used to estimate the effect of scatter correction in MSP timing. We discuss the mathematical formulation of scattering and deconvolution methods in more detail in Chapter 3.

\subsection{Pulsar data processing}

Since pulsars are weak radio sources, they are generally observed across a wide frequency band that could be as large as $800 \mathrm{MHz}$. The signal sampled by a wave-guide feed to orthogonal polarizations and is split into frequency channels using a spectrometer. Raw data are recorded either as filterbank data, where the phase information of the signal is not recorded, or using baseband devices, which record the full incoming signal. The key procedures involved in data processing are dedispersion, where the signal is corrected for dispersion from the ISM and folding, 
where the time series is averaged over a few minutes or hours to form a stable profile from single pulses which are buried in noise. In situations where the pulsar is sufficiently bright so that single pulses are also detectable, many unique experiments can be performed to extract information that may not be available in the average folded profiles. Radio frequency interference (RFI) removal and calibration for flux and polarization are also important next steps in pulsar data processing.

\subsubsection{Dedispersion}

Correcting for ISM dispersion delays can be performed either as incoherent or coherent dedispersion. Incoherent dedispersion applies the appropriate time delay to each frequency channel, calculated using Equation 1.15, so that the pulses arrive at the same time throughout the frequency band. This method is limited by the width of the frequency channels. Coherent-dedispersion on the other hand, assumes that the effect of the ISM is the convolution of the pulsar voltage signal and a transfer function with a frequency dependent phase, which represents the ISM delays. The originally emitted voltage signal is recovered via deconvolution. Therefore, coherent dedispersion, which has the ability to completely remove dispersion delays, is currently more useful for high precision timing experiments.

\subsubsection{Obtaining single pulses and folded profiles}

When a pulsar is first discovered, the spin frequency is obtained from the peak position of the Fourier transform of the dedispersed time series. To obtain profiles 
averaged over many rotations, the time series is folded using the spin period. For newly discovered pulsars, folding is done using the initial spin period obtained from the Fourier transform. After a few observations, a more precise measurement for the spin period may be obtained though timing the pulsar, which will be discussed in detail in Section 1.6.

The time resolution needed for single pulse searches is determined by dispersion smearing, dedispersion error, the filter response of a frequency channel and broadening due to multipath scattering (Cordes and McLaughlin 2003). Single pulses are searched for in a time series as pulse amplitudes that exceed the root-mean-square (RMS) noise level by several standard deviations, typically $5 \sigma$. In the case of intermittent pulsars such as RRATs, the period is calculated as greatest common divisor of every interval between the pulses. This period is used to fold the time series data to produce an average pulse profile.

\subsubsection{Radio frequency interference removal}

Radio frequency interference (RFI) may appear as both narrowband and broadband in frequency. Furthermore, it could be pulsed, bursts or continuous signals. There are multiple ways in which RFI can be removed from data. In the case of narrowband RFI, one straightforward method is to set the affected channels and sub integrations to zero using routines within PSRCHIVE (Hotan et al., 2004) such as paz. This technique was used when processing the single-pulse data as described in Section 4.2. In the time domain, broadband signals are usually identified in the 
zero DM time series, because these terrestrial signals do not undergo dispersion due to the ISM. When processing single-pulse data, an automated RFI removal method may increase the $\mathrm{S} / \mathrm{N}$. In the case of narrowband RFI, one such method is median zapping where all channels with total flux that differs from the median-smoothed spectrum by more than four times the standard deviation are set to zero. In the case of broadband RFI all off-pulse phase bins with total flux that differs from the median-smoothed profile averaged over frequency by more than four times the standard deviation are set to zero. This is done before dedispersing the data. See van Straten et al. (2012) for more details on RFI excision.

\subsubsection{Flux calibration}

The radiometer equation expresses the root mean square fluctuations in the system temperature $T_{\text {sys }}$ as

$$
\Delta T_{\text {sys }}=\frac{T_{\text {sys }}}{\sqrt{n_{p} t_{\text {obs }} \Delta f}}
$$

where $\Delta f$ is the observing bandwidth, $n_{p}$ is the number of polarizations and $t$ is the observing time.

The mean flux of the pulsar can be expressed as

$$
S_{\text {mean }}=\frac{\mathrm{S} / \mathrm{N} \mathrm{T}_{\mathrm{sys}}}{G \sqrt{n_{p} t_{\text {obs }} \Delta f}} \sqrt{\frac{W}{P-W}},
$$

where $P$ and $W$ are the pulsar period and pulse width respectively. Flux calibration 


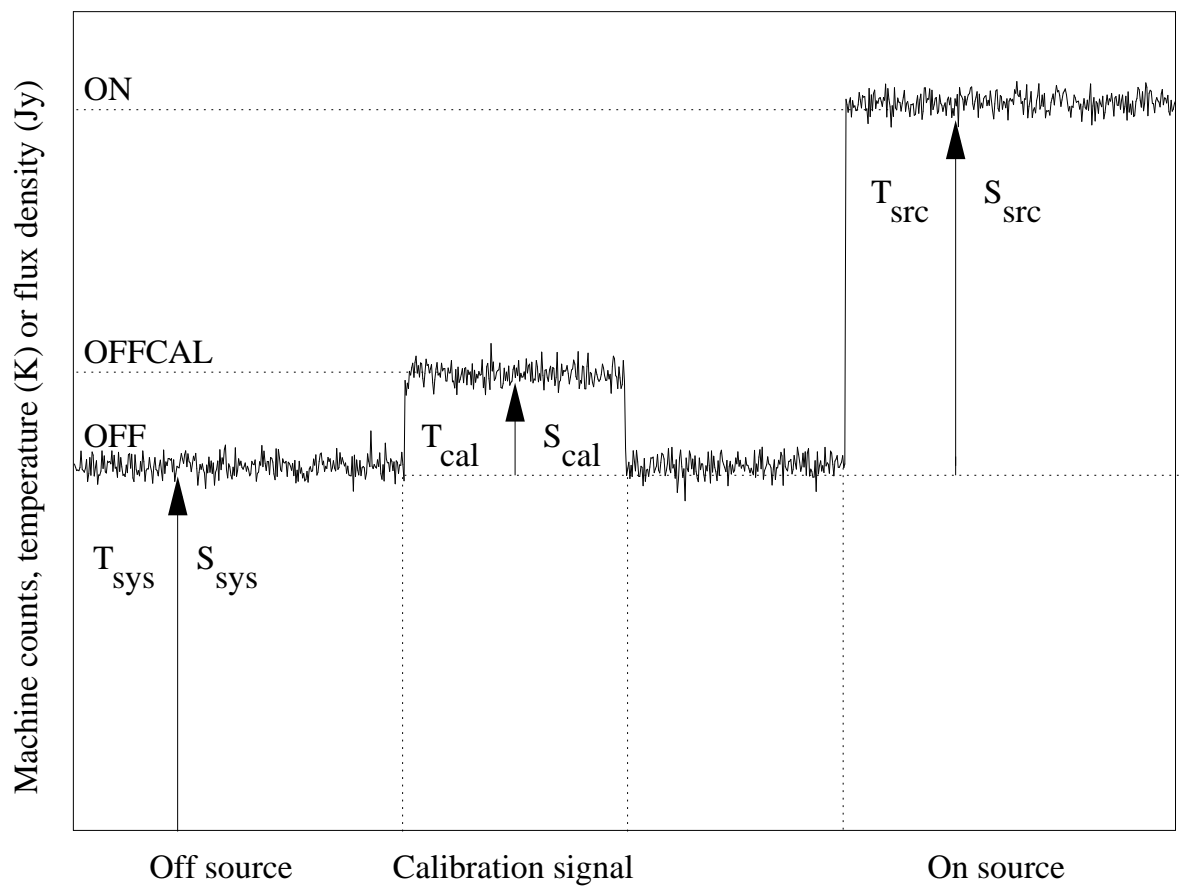

Figure 1.5: The relationship between antenna temperature and flux density used in calibration (Lorimer \& Kramer, 2005). The noise in $T_{\text {sys }}$ shown in the figure has root mean square fluctuations of $\Delta T_{\text {sys }}$ and $\mathrm{S} / \mathrm{N} \propto T_{\text {peak }} / \Delta T_{\text {int }}$, where $T_{\text {peak }}$ is the peak amplitude of a pulse and $T_{\text {int }}$ is the quadrature sum of $\Delta T_{\text {sys }}$ in the on and off states.

is necessary in order to obtain accurate flux measurements given that the telescope records data in machine units instead of flux. There are multiple ways the pulsar flux can be estimated. The simplest method, in the case where a calibrator is not observed, is to estimate the flux from the system noise $\Delta S_{s y s}$. Using the relationship $S_{s y s}=T_{s y s} / G$ in the radiometer equation in Equation 1.16 we obtain,

$$
\Delta S_{s y s}=\frac{T_{\text {sys }}}{G \sqrt{n_{p} t_{\text {obs }} \Delta f}} .
$$

In this case, observed intensity can be converted to flux units by multiplying by $\Delta S_{\text {sys }} / \sigma$, where $\sigma$ is the off pulse RMS. This method is subject to an uncertainty of $\sim 25 \%$ due to variations in the system temperature and the usable bandwidth. 
The other methods involve observing a calibrator, usually a quasar with known flux $S_{s r c}$. Figure 1.5 shows the relationship between machine units and flux density for a typical observing system. In this case, the system temperature $T_{\text {sys }}$ is calculated directly from the ON and OFF source observations where the telescope observes the source and a nearby patch of sky with no sources $\sim 1^{\circ}$ away from the source respectively, as

$$
\frac{T_{s r c}}{T_{s y s}}=\frac{\mathrm{ON}-\mathrm{OFF}}{\mathrm{OFF}},
$$

where $T_{s r c}=G S_{s r c}$.

A more accurate method is to inject a noise signal and calculate its flux using the flux measurement of the calibrator source. This way the assumption that $T_{\text {sys }}$ remains the same for both $\mathrm{ON}$ and OFF source observations can be avoided. In this case, using Figure 1.5 and the same idea as before, we can say that,

$$
\frac{T_{c a l}}{T_{s y s}}=\frac{\mathrm{OFFCAL}-\mathrm{OFF}}{\mathrm{OFF}}
$$

where OFFCAL is when the noise diode is added during the off-source pointing. This can be combined with Equation 1.19 which gives,

$$
\frac{S_{c a l}}{S_{s r c}}=\frac{\mathrm{OFFCAL}-\mathrm{OFF}}{\mathrm{ON}-\mathrm{OFF}}
$$

This can be used to establish a flux density scale for pulsar observations by calculating $S_{c a l}$, using the known flux of the calibrator $S_{s r c}$. 


\subsubsection{Polarization calibration}

The Stokes parameters can be expressed in terms of the $X$ and $Y$ components of the electric field as

$$
\begin{aligned}
& I=E_{x}^{2}+E_{y}^{2} \\
& Q=E_{x}^{2}-E_{y}^{2} \\
& U=2 E_{x} E_{y} \cos \phi \\
& V=2 E_{x} E_{y} \sin \phi,
\end{aligned}
$$

where $E_{x}$ and $E_{y}$ are the $X$ and $Y$ components of the E-field and $\phi$ is the relative phase between the two components.

The state of polarization of radiation from the source undergoes changes in amplitude and phase due to effects such as Faraday rotation, parallactic angle rotation and instrumental effects. These can be classified into changes due to the feed rotation with respect to the sky, the feed itself, imperfections in the feed and imperfections in the amplifier chains. See Heiles et al. (2001) for a complete review of these effects. The modification of the intrinsic stokes parameters $S_{\text {int }}$ can be described by a $4 \times 4$ Mueller matrix $M$, such that the observed Stokes parameters are given by

$$
S_{o b s}=M S_{\text {int }} .
$$

The feed may mix the incoming linear polarization to any degree of elliptical polarization. This is described by the amount of coupling $\alpha$ into the orthogonal polar- 
ization state, and $\chi$, the phase angle of coupling. For a dual linear feed, $\alpha=0^{\circ}$ and $\chi=0^{\circ}$. For a dual circular feed, $\alpha=45^{\circ}$ and $\chi=90^{\circ}$. Additionally receptors may not be ideal linear or circular, and their orientation may not be exactly orthogonal. These imperfections could introduce unwanted coupling between the orthogonal polarizations $X$ and $Y$, and can be described by $\epsilon$ and $\phi$ representing the amplitude and phase of the cross coupling.

Next, the orthogonal signals may travel through different amplifier chains, which introduce a phase delay due to different path lengths between the two components, and also changes in the voltage gain, leading to a change in the polarization state. The two cables will change the amplitudes and phases of the two orthogonal components differently. These voltage gains can be described by $g_{x}$ and $g_{y}$ and the phase delays by $\psi_{x}$ and $\psi_{y}$ for the $X$ and $Y$ components of the Electric field. From these quantities the differential gain and differential phase are defined as $\Delta G=G_{X}-G_{Y}$ and $\psi=\psi_{x}-\psi_{y}$, where $G=g^{2}$.

This gives instrumental parameters $\Delta G, \psi, \epsilon, \phi, \alpha$ and $\chi$ to be solved for. In addition there is the rotational parameter which represents the rotation about the line of sight by the parallactic angle, as the line-of-sight sweeps past the magnetic field lines in the emission beam, as explained in Section 1.5. In addition to the intrinsic position angle of the source, Faraday rotation due to the ISM and the ionosphere, and the parallactic angle of the feed also contribute to the rotational parameter. The Mueller matrix $M$ in Equation 1.23 consists of these parameters and needs to be solved in order to determine the intrinsic polarization of the pulsar $S_{\text {int }}$. These unknowns can be better determined by measuring the pulsar Stokes 
parameters at different parallactic angles. To simply things further, the amplifier gains and phases are calibrated using a cal signal, which is basically a fully linearly polarized signal produced by the noise diode in the receiver, and injected between the two probes at $45^{\circ}$. That is, the Stokes parameters of the pulsar measured at multiple pulse longitudes on multiple observations are used to constrain the above instrumental parameters and the model Stokes parameters of the source, noise diode and the flux calibrator.

PSRCHIVE (Hotan et al., 2004) pac does the first order calibration with the ideal feed assumption (no ellipticities, orthogonal receptors). In reality, however, the cal signal may have a circularly polarized component, and its position angle may not be exactly $45^{\circ}$.

\subsection{Pulsar timing}

Pulsars are stable rotators. A TOA refers to a the arrival time of the peak of the profile based on the recorded time of the first sample of the observation. The precise measurement of the arrival time of pulses is key to many experiments performed using pulsars.

A TOA of a pulse profile is calculated via a Fourier domain $\chi^{2}$ minimization technique between the observed pulse profile and a template profile (Taylor, 1992). In this framework, the observed profile $y(t)$ is expected to be a shifted and scaled version of the standard profile $s(t)$. The standard profile is usually obtained by generating a high $\mathrm{S} / \mathrm{N}$ profile by averaging over many observations. In this method 
the observed average profile is assumed to be related to the standard Gaussian modulation function (Taylor, 1992) as

$$
y(t)=a+b s(t-\tau)+g(t)
$$

where $a, b$ and $\tau$ are constants, and $g(t)$ is a random noise variable. The best estimate for the time offset $\tau$ is found through a $\chi^{2}$ minimization of the observed profile and the standard profile.

For a profile with a width of $\mathrm{W}$, the error on the TOA due to radiometer noise is given by $\delta T O A \sim W /(\mathrm{S} / \mathrm{N})$, where $\mathrm{S} / \mathrm{N}$ is the signal-to-noise ratio of the profile. Pulsar timing allows pulse periods to be measured with an accuracy of $\delta P=\delta T O A / \Delta T$, where $\Delta T$ is the time span of observation. For a typical MSP, whose TOA error can be as low as $\sim 0.1 \mu \mathrm{s}$, and observed for several years, the pulse period can be calculated with a precision of $\sim 10^{-16}$ s. Once the TOAs are obtained using this template-matching technique, the pulsar can be timed, to derive various parameters by a least-square optimization of a pulsar model.

In the pulsar model, the time-dependent phase of the pulse $\phi(T)$ as a function of the barycentric time $T$ is given through Taylor expansion as

$$
\phi(T)=\phi_{0}+\left(T-T_{0}\right) \Omega_{0}+\frac{1}{2}\left(T-T_{0}\right)^{2} \dot{\Omega}_{0}+\ldots
$$

where $T_{0}$ is some reference epoch, $\Omega_{0}$ is the modeled rotational frequency and $\phi_{0}$ is the phase at epoch $T_{0}$. Given a timing model that accounts for parameters such as 

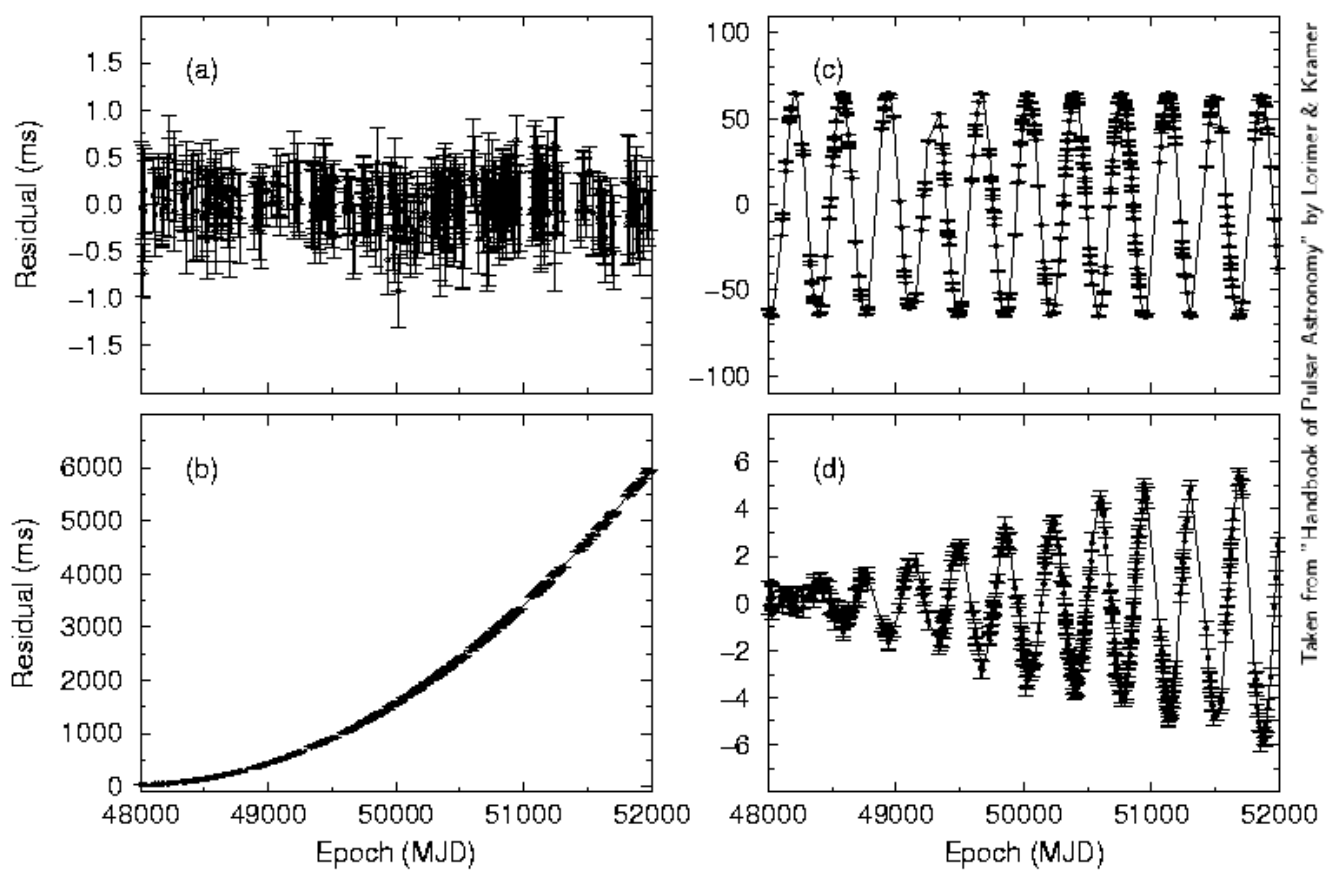

Figure 1.6: (a) Timing residuals of a slow pulsar for a correct timing solution showing a random distribution around a mean of zero. (b) Systematic increase in residuals due to an improper value of $\dot{P}$. (c) Variation in residuals due to an incorrect position. The sinusoidal nature, with a period of one year, is due to the motion of the Earth around the Sun. (d) Residuals due to neglecting the pulsar's proper motion. Figure used with permission from the Handbook of Pulsar Astronomy (Lorimer \& Kramer, 2005).

pulsar period, period derivative, position, proper motion and orbital parameters if needed, the differences between measured and model TOAs, called timing residuals, are calculated. The best-fitting model parameters are obtained by minimizing the RMS of residuals. Software packages TEMPO ${ }^{2}$ and TEMPO2 (Hobbs et al., 2006) are used for timing analysis.

Errors in the model, for example in the pulse period, period derivative or position, will result in noticeable effects in the timing residuals. For example, an error in the period will result in a linear trend in time as given by Equation 1.25. While an error in the period derivative will result in a quadratic trend, an error in

\footnotetext{
${ }^{2} \mathrm{http}: / /$ tempo.sourceforge.net
} 
the position will result in a sinusoidal trend and an error in the proper motion will result in a sinusoidal trend that amplifies with time in the residuals. These trends are shown in Figure 1.6.

\subsection{Using millisecond pulsars to detect gravitational waves}

The remarkable stability of MSPs make them excellent tools to test fundamental physics occurring in stellar environments that cannot be performed inside laboratories on earth. One such application is the detection of gravitational waves (GWs). Gravitational waves are a key prediction of Einstein's theory of general relativity and their existence has been supported through timing measurements of the orbital decay of the Hulse-Taylor binary system B1913+16 (Hulse \& Taylor, 1975). Many experiments aim to detect these waves directly through the measurement of light travel changes between objects. Complementary to the sensitivity of interferometer-based GW detection experiments like LIGO, pulsar timing arrays (PTAs) are sensitive to nanohertz frequency GWs.

GWs are ripples in the curvature of spacetime, generated by accelerating masses. Detectable levels of gravitational radiation are produced when the masses involved are as large as neutron stars for ground based detectors like LIGO or supermassive binary black holes (SMBBH) for PTAs. By observing on timescales of T years, PTAs aim to detect GWs in the nanohertz frequencies $(1 / \mathrm{T})$ through precision timing of millisecond pulsars. SMBBHs with orbital periods of years, which generate nanohertz frequency gravitational waves, fall naturally to the regime de- 
tectable by millisecond pulsar timing. The timing residuals will be correlated with sky position among pulsars distributed across the sky (Hellings \& Downs, 1983). While PTAs are sensitive to a stochastic gravitational wave background (GWB), generated by a population of SMBBHs, cosmic strings (Damour \& Vilenkin, 2005) and inflation (Turner, 1997), strong signals from individual binaries may be detectable (Sesana et al., 2012).

Under the simplest assumptions, the GWB is expected to be a power-law spectrum with a spectral index of $\alpha=-2 / 3$ (Jaffe \& Backer 2003). PTAs are sensitive to sources located nearby, at a redshift of $0.2<z<2$ (Sesana et al 2009), with large masses of around $M \sim 10^{9} M_{\odot}$ and that are compact, with orbital periods of around $\sim 0.1-10 \mathrm{yr}$. This corresponds to separations of milli-pc scales. The limits on the redshift correspond to the smaller volume at low redshifts and GW signal attenuation and decrease in the merger rate at high redshifts. In addition to the search for a GWB, PTAs also search for continuous waves and GW bursts in the timing data.

In order to detect the background due to supermassive black hole binaries, over 40 MSPs with root-mean-square (RMS) timing residuals of less than $100 \mathrm{~ns}$ are likely required (Jenet et al., 2005; Cordes \& Shannon, 2012). Currently over 40 millisecond pulsars are being timed by the North American Nanohertz Observatory for Gravitational waves (NANOGrav), with RMS timing residuals of nearly all pulsars at the sub-microsecond level (Demorest et al., 2013; McLaughlin, 2013). In addition to GWs, other effects such as ISM propagation and rotational irregularities will affect the arrival times of pulses. Fortunately, ISM effects are chromatic and 
therefore multi-frequency observations can be used to at least partially correct for these variations.

We discuss mitigation techniques for ISM scattering as applicable to PTAs in Chapter 3 and discuss other noise sources affecting the TOAs, such as variable polarization properties and pulse-to-pulse jitter in Chapter 4. 


\title{
Chapter 2
}

\section{Radio Properties of Rotating Radio Transients}

Published in MNRAS (Palliyaguru et al., 2011), with co-authors $M$.

\author{
A. McLaughlin, E. F. Keane, M. Kramer, A. G. Lyne, D. R. Lorimer, \\ R. N. Manchester, F. Camilo and I. H. Stairs
}

\subsection{Introduction}

Rotating Radio Transients (RRATs) are neutron stars which were discovered only through their isolated pulses (McLaughlin et al., 2006). Some, however, have later been detectable through periodicity searches. The average intervals between detected pulses range from a few minutes to a few hours and pulses have durations between 2 and 30 ms. Thus far, 90 RRATs have been identified (Hessels et al., 2008; Deneva et al., 2009; Keane et al., 2009; Burke-Spolaor \& Bailes, 2010b; Keane et al., 2011; Burke-Spolaor et al., 2011), including the original 11 from McLaughlin et al. (2006). Periods ranging from 0.1 to 8 seconds have been measured for 67 of these sources. Period derivatives have been measured for 24, allowing inference of spin-down properties such as characteristic ages and surface dipole magnetic fields (McLaughlin et al., 2009; Lyne et al., 2009; Keane et al., 2011). The periods and magnetic fields of RRATs are larger than those of normal pulsars, but the distributions of other spin-down properties such as spin-down energy loss rate and 
characteristic age are similar (McLaughlin et al., 2009). Despite this overall trend, the properties of individual RRATs vary considerably. Four RRATs, including PSRs J1826-1419 and J1913+1330, have spin-down properties consistent with the bulk of the non-recycled radio pulsar population and two others, PSRs J1317-5759 and J1444-6026, have properties similar to non-recycled, older pulsars. Four others, PSRs J1652-4406, J1707-4417, J1807-2557, and J1840-1419, lie just above the radio 'death-line' (e.g. Chen \& Ruderman, 1993; Zhang et al., 2007). However, some have more unusual spin-down properties. PSRs J0847-4316, J1846-0257, and J1854+0306, lie in an empty region of $P-\dot{P}$ space between the normal radio pulsars and isolated neutron stars (XINS) and PSR J1819-1458 has a high magnetic field of $5 \times 10^{13} \mathrm{G}$. See the $P-\dot{P}$ diagram in Figure 1.4.

Because of the difficulties in detecting these sporadic objects, the total Galactic population of RRATs likely outnumbers that of normal radio pulsars (McLaughlin et al., 2006), though it is possible that the populations are evolutionarily related (Keane \& Kramer, 2008). Several ideas have been presented about the nature of the emission from these objects. It could be similar to that responsible for the 'giant pulses' observed from some pulsars (e.g. Knight et al., 2006). It could also be that the sporadic emission is related to the fact that these objects are near the radio 'death-line' (e.g. Chen \& Ruderman, 1993; Zhang et al., 2007) and/or are examples of extreme nulling (e.g. Redman \& Rankin, 2009). The phenomenon has also been attributed to the presence of a circumstellar asteroid belt (Li, 2006; Cordes \& Shannon, 2008) or a radiation belt as seen in planetary magnetospheres (Luo \& Melrose, 2007). Or, perhaps, some are transient X-ray magnetars (e.g. Woods et al., 
2005). Another idea is that their properties lie at the extreme end of the population of normal radio pulsars. Weltevrede et al. (2006) show that PSR B0656+14, a nearby middle-aged pulsar which emits pulses with energies many times its mean pulse energy which are different from giant pulses due to the larger widths, would be discovered as a RRAT source if it were farther away. RRATs may also be considered as an extreme case of mode changing (see, e.g., Wang et al., 2007) where the on state is less than or about one pulse period. Furthermore, Lyne et al. (2010) have recently shown that many pulsars exhibit a two-state phenomenon in which varying pulse profile shapes are correlated with variations in spin-down rates and implied changes in magnetospheric particle density. These changes are quasi-periodic, with timescales ranging from one month to many years. It could be that the RRATs are similar two-state systems, in which the profile changes are so dramatic to make them undetectable in the more common state.

Determining the time variability and/or periodicity of the RRAT pulses is therefore an important diagnostic of the RRAT emission mechanism. While the pulse profile, and, in most cases, pulse intensity changes of Lyne et al. (2010) are quasi-periodic, the pulse intensity distributions of normal pulsars and giant-pulsing pulsars are believed to be random over time. On the other hand, nulling pulsars in general show on and off timescales of more than one consecutive pulse. A study by (Redman \& Rankin, 2009) shows that a majority of nulling pulsars show largely non-random distributions. Radio emitting neutron stars including pulsars but also radio magnetars often show transient spin-down phenomena as well. For instance, glitches, or sudden increases in the spin frequency, have been observed 
from young pulsars and one RRAT ( PSR J1819-1458). One of the glitches from PSR J1819-1458 was accompanied by a $3.5 \sigma$ increase in the pulse detection rate (Lyne et al., 2009). Radiative events such as enhanced fluxes or detection rates do not normally accompany the glitches of normal radio pulsars, but are quite common for magnetars (Dib et al., 2008a). This, along with the high magnetic field of PSR J1819-1458, hints at a relationship with magnetars and also provides additional motivation to examine the pulse rate variations with time for all RRATs.

We search for periodicities and quantify the randomness of the detected RRAT pulses in several different ways. We first search for periodicities in the pulse arrival times on minutes-year long time scales and pulse detection rates on month-year long time scales using a Lomb-Scargle analysis. We then quantify the randomness of the RRAT pulse arrival times using Kolmogorov-Smirnov tests on seconds-year long time scales. The observations are described in Section 2.2, the methods and results in Section 2.3, and the conclusions and plans for future work in Section 2.4.

\subsection{Observations}

All eight sources discussed in this paper were discovered by McLaughlin et al. (2006) in a re-analysis of data from the Parkes Multi-beam Pulsar Survey (PMPS).

We have ignored three of the original 11 RRATs as their pulse detection rates are too low to perform this analysis. The discovery data were taken between Jan 1998 and Feb 2002 and follow-up observations began in Aug 2003 and are ongoing using the 64-m Parkes telescope. Most of the observations used the central beam of the 
multi-beam receiver with a central frequency of $1.4 \mathrm{GHz}$ and a bandwidth of 256 MHz. A few observations used other frequencies; we ignore these in our analysis to ensure uniformity of pulse detection rates. The sources have been observed at between 27 and 89 epochs at $1.4 \mathrm{GHz}$, with each observation $0.5-2 \mathrm{hr}$ in duration (see Table 2.1).

One important consideration in our analysis is the influence of the interstellar medium on our observed pulses. The scintillation bandwidth for diffractive scintillation can be estimated as $\Delta \nu_{D I S S} \propto \nu^{4.4} d^{-1}$, where $\nu$ and $D$ are the observing frequency and the distance to the pulsar. Assuming a pulsar velocity of $100 \mathrm{~km} \mathrm{~s}^{-1}$, the diffractive timescales $\Delta t_{D I S S}$ can be estimated. The refractive timescales can then be calculated as $\Delta t_{R I S S}=\nu \Delta t_{D I S S} / \Delta \nu_{D I S S}$. The modulation index of scintillation, defined as the ratio between the standard deviation of the observed flux densities and their mean, can also be given by $m=\left(\Delta \nu_{D I S S} / \nu\right)^{1 / 6}($ Lorimer \& Kramer, 2005). For all of these sources, the predicted diffractive scintillation bandwidths at 1.4 GHz are less than $1 \mathrm{MHz}$ (Cordes \& Lazio, 2002), making modulation due to diffractive scintillation unimportant. In Table 2.1, we list the predicted timescales for refractive scintillation at our observing frequency of $1.4 \mathrm{GHz}$, estimated from the predicted diffractive scintillation timescales and bandwidths from Cordes \& Lazio (2002) (see, e.g., Lorimer \& Kramer (2005)). These timescales range from 21 to 197 days. However, the actual timescales could vary significantly from those predicted. The predicted modulation indices due to refractive scintillation (Lorimer \& Kramer, 2005) range from 0.09 to 0.17 , meaning these are expected to be relatively minor contributions to pulse rate variations. 


\subsection{Analysis and Results}

Pulse detection is performed by dedispersing the data at the dispersion measure (DM) of the RRAT and at a DM of zero. Then pulses are searched for in both time series above a $5 \sigma$ threshold using the pulsar processing package SIGPROC $^{1}$. Pulses which are brighter at the DM of the RRAT are likely to be from the source. We inspect the pulses visually by checking for pulse shape and pulse phase consistency to be certain of their astrophysical nature. For some epochs which have large amounts of radio frequency interference, we applied the above procedure but with multiple trial DMs as described by McLaughlin et al. (2009). If more than one pulse is detected within an observation, a second check based on the known period of the source can be made by requiring that all pulses have arrival times which differ by integer multiples of the period. For the sources with phase-connected timing solutions, we check that the pulse arrival time is consistent with the solution. In Table 2.2, we list the number of epochs for which pulses were detected for all sources. In Table 2.3 we list the total number of pulses detected within the entire time span of observations.

\subsubsection{Periodicity search}

The Lomb-Scargle test (Scargle et al. 1982) is a statistical procedure for uncovering periodic signals hidden in noise. We use this technique in our analysis as our data are unevenly sampled, thereby making standard Fourier analysis difficult.

\footnotetext{
${ }^{1}$ http://sigproc.sourceforge.net
} 
The implementation we used (Numerical Recipes, see Press et al. (1986)) utilizes a version of the periodogram with modifications by Scargle (1982) and Horne and Baliunas (1986). Applications of this test to radio pulsar data can be found in Bailes et al. (1997) and Kramer et al. (2006).

The Lomb-Scargle test reveals signals in the power spectral density distribution of a source, with the presence of a sinusoid of certain frequency indicated by a peak in the spectrum at that particular frequency. The trial frequencies at which the periodogram is evaluated are chosen to be a finite evenly spaced set. For a time series $X\left(\mathrm{t}_{\mathrm{i}}\right)$ with $N_{0}$ number of elements where $\mathrm{i}=1,2, \ldots N_{0}$, the Scargle angular frequencies range from $\omega=2 \pi / T$ to $\omega=\pi N_{0} / T$ (or periods from $T$ to $2 T / N_{0}$ ), where $T$ is the total time interval. The searched frequencies therefore range up to the Nyquist frequency. The number of frequencies searched is obtained from the empirical formula (Horne and Baliunas 1986)

$$
N_{i}=-6.362+1.193 \times N_{0}+0.00098 \times N_{0}^{2}
$$

The likelihood of the existence of a signal or the level of significance is calculated as a detection threshold $Z_{0}$ (Scargle 1982),

$$
Z_{0}=-\ln \left[1-\left(1-p_{0}\right)^{1 / N_{i}}\right]
$$

The false alarm probability $p_{0}$ is the probability that a peak of power $Z_{0}$ will occur in the absence of a periodic signal. 


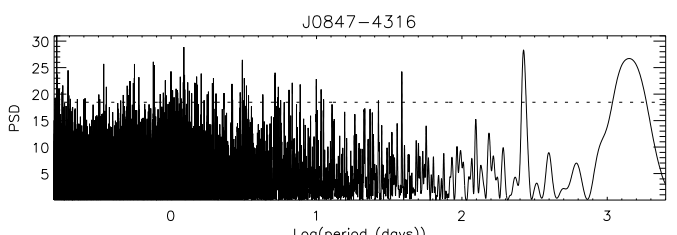

Log(period (days))
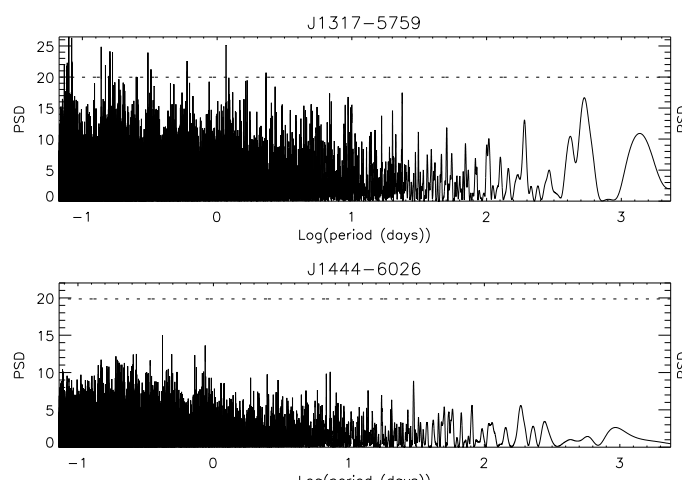

Log(period (days))

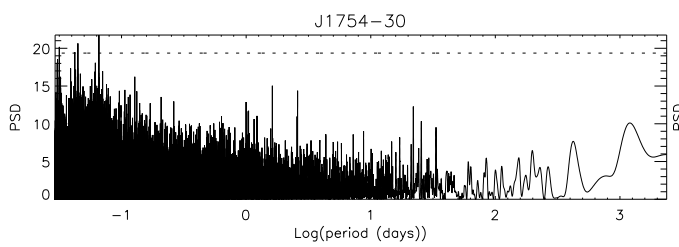

J1819-1458

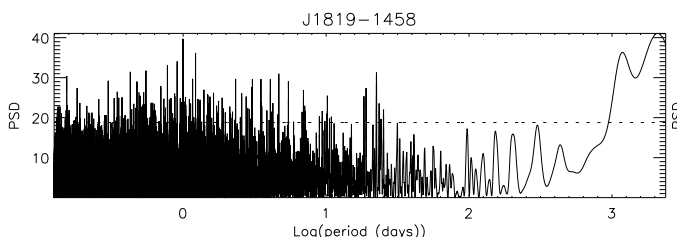

$\log ($ period (days)
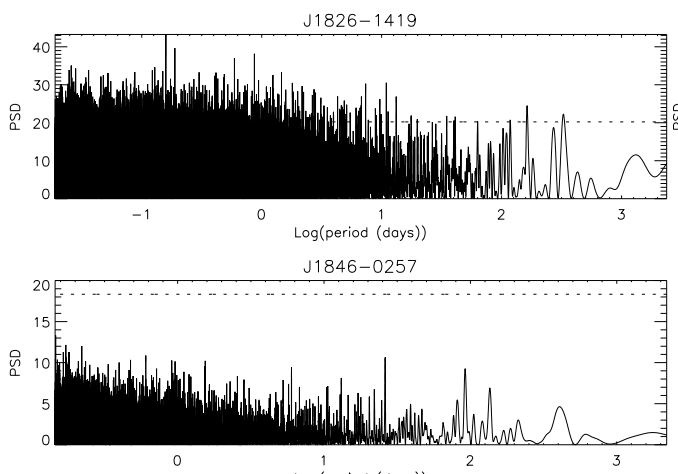

$\log ($ period (days))

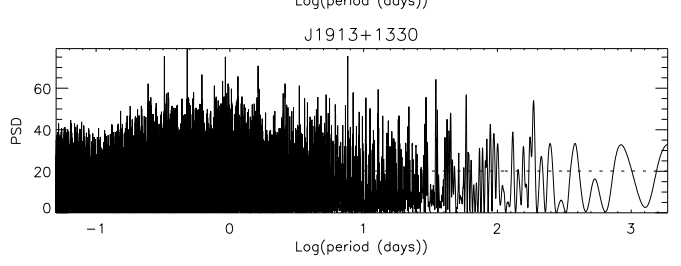

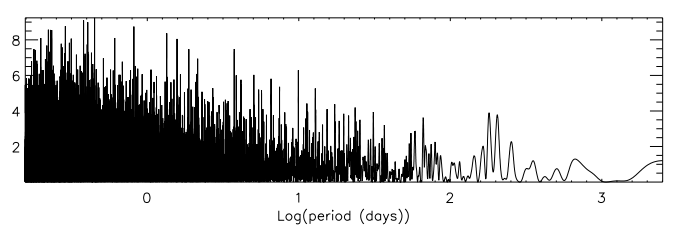

$\log ($ period (days))
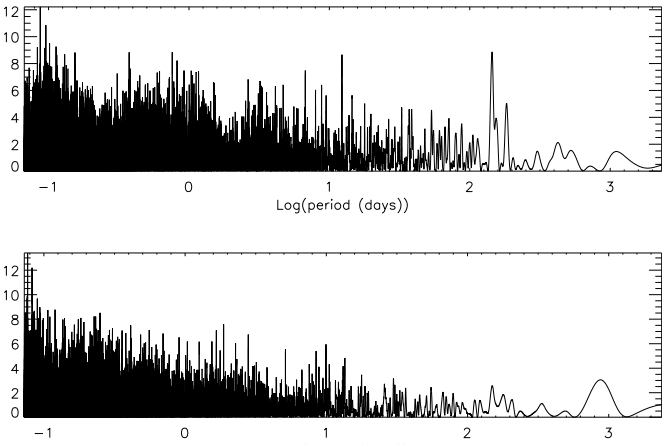

$\log ($ period (doys))
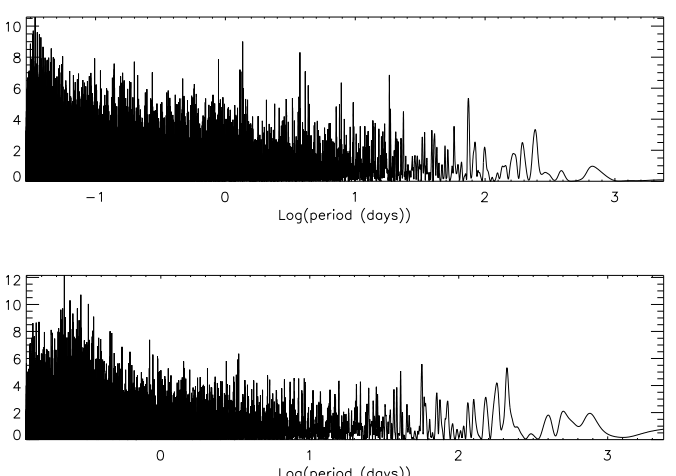

$\log ($ period (days))
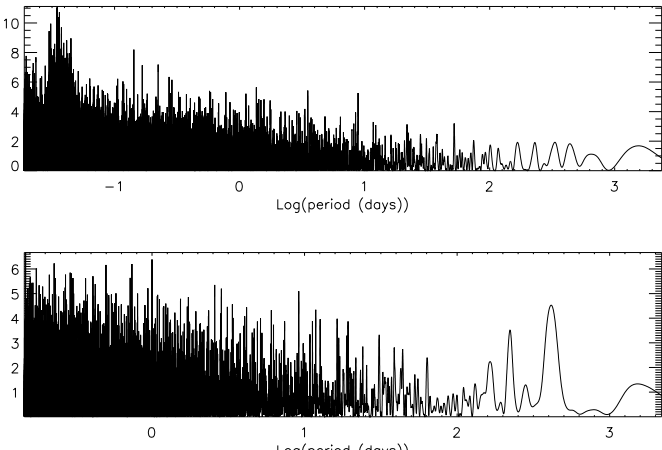

$\log ($ period (days))

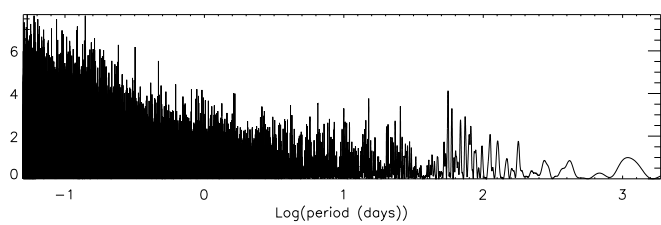

Figure 2.1: Power spectral density vs. period from the Lomb-Scargle analysis on pulse arrival times for PSRs J0847-4316, J1317-5759, J1444-6026, J1754-30, J1819-1458, J1826-1419, J1846-0257, and J1913+1330 (left panel) and the corresponding plots when the arrival times are randomized (right panel). The randomized time series do not show significant peaks in the spectra. The dashed line represents the $99 \%$ significance level. Note the different y-axis scales in the left and right panels. 

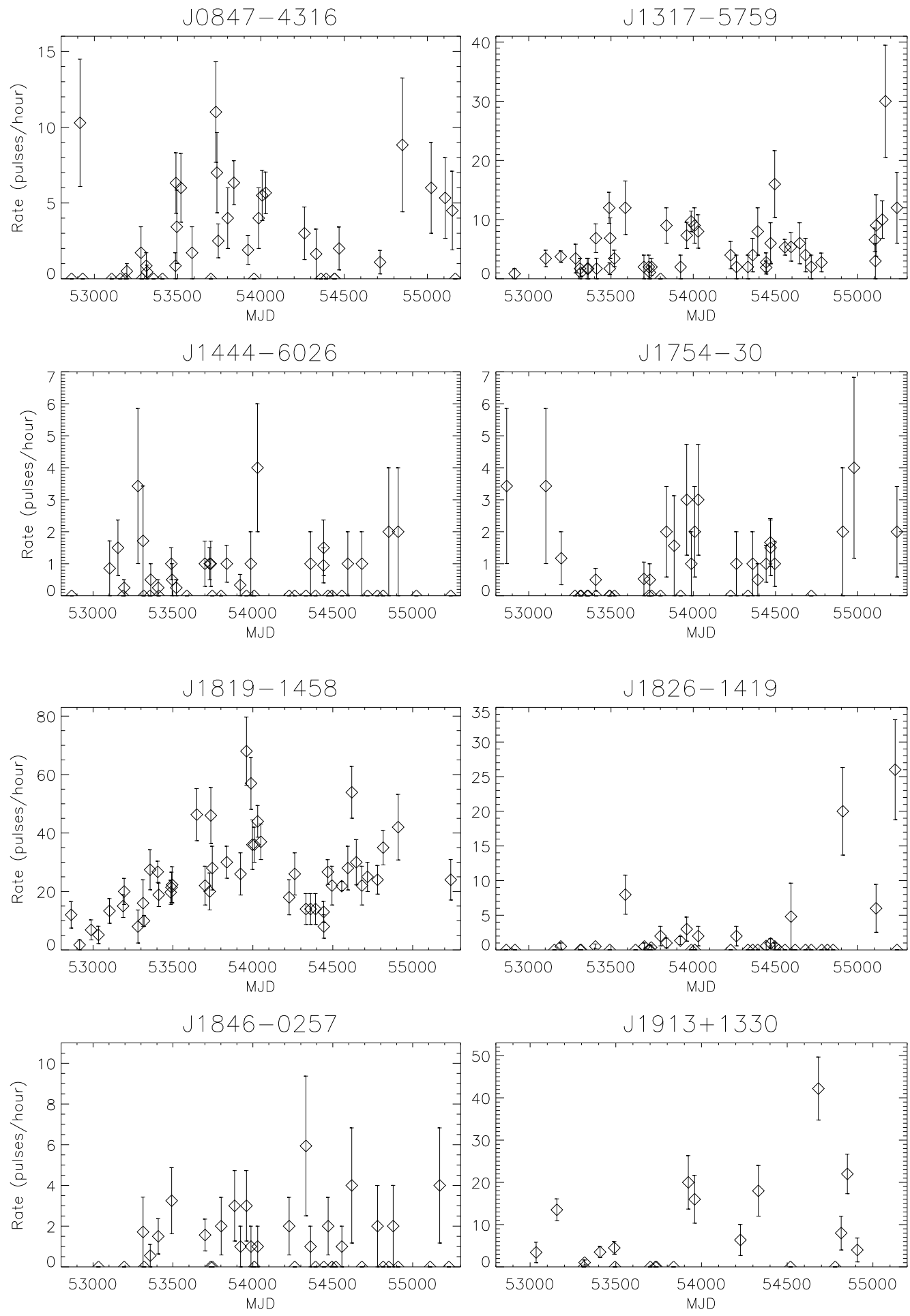

Figure 2.2: Daily pulse detection rate vs. date for PSRs J0847-4316, J1317-5759, J1444-6026, J1754-30, J1819-1458, J1826-1419, J1846-0257, and J1913+1330. The errors on the rates are calculated as $\sqrt{(} N) / T$, where $N$ and $T$ are the number of pulses and the observation length respectively. 

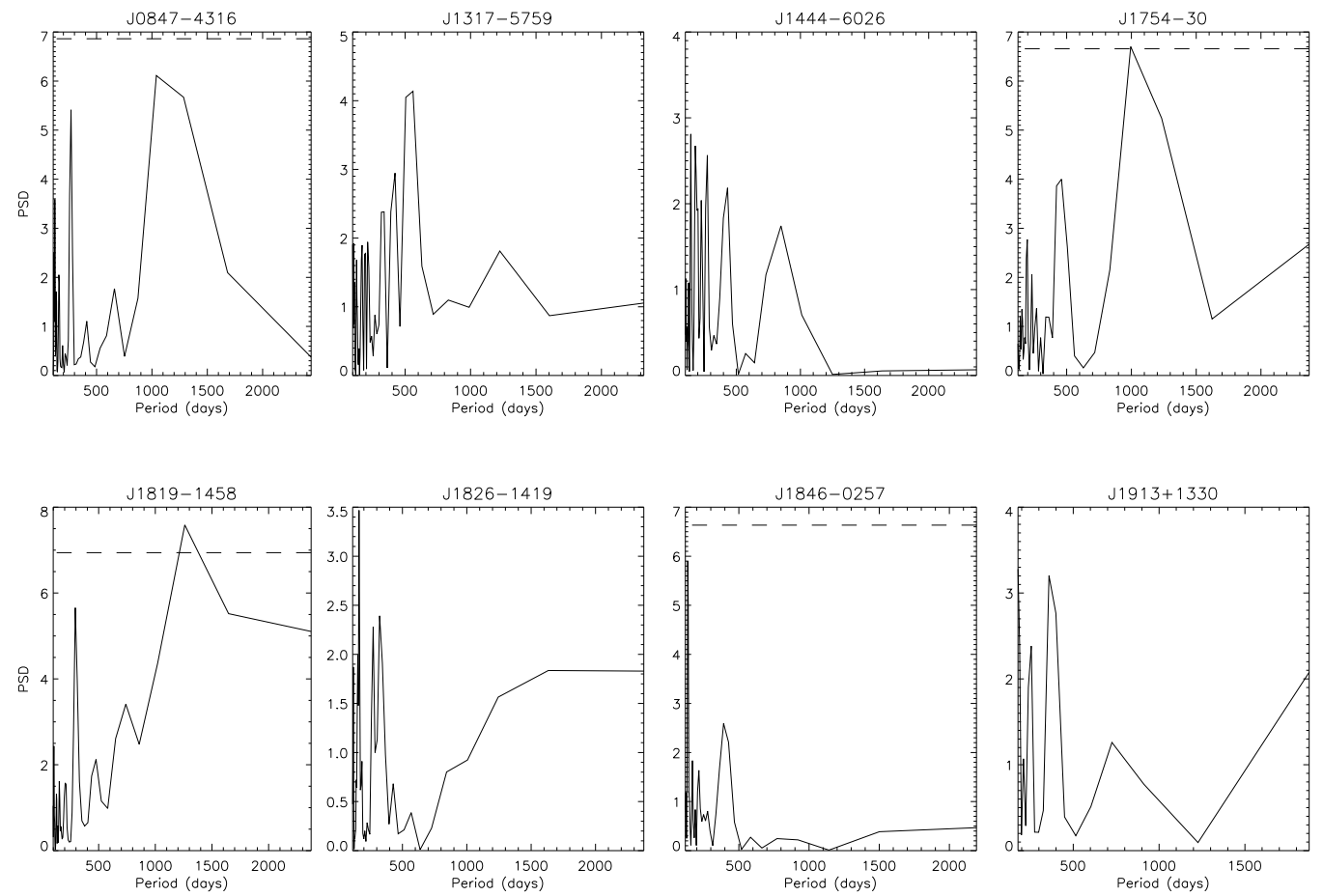

Figure 2.3: Power spectral density vs. period from the Lomb-Scargle analysis on daily pulse detection rates for PSRs J0847-4316, J1317-5759, J1444-6026, J1754-30, J1819-1458, J1826-1419, J1846-0257, and J1913+1330. The dashed line represents the $95 \%$ significance level.

Table 2.1: Spin period, distance, time between the first and last epochs of observation, the number of epochs, fluctuation period of the pulse detection rate, the significance of the most prominent peak in the spectrum, predicted refractive scintillation timescale (using diffractive scintillation timescales and bandwidths from Cordes \& Lazio (2002)), predicted modulation index due to refractive scintillation, and base-10 logarithms of the derived parameters characteristic age and surface dipole magnetic field strength.

\begin{tabular}{lllllllllll}
\hline $\begin{array}{l}\text { Name } \\
\text { PSR }\end{array}$ & $\begin{array}{l}\text { Period } \\
(\mathrm{sec})\end{array}$ & $\begin{array}{l}\text { Distance } \\
(\mathrm{kpc})\end{array}$ & $\begin{array}{l}\text { Data Span } \\
(\text { days })\end{array}$ & $\begin{array}{l}\mathrm{N}_{\mathrm{e}} \\
(\text { days })\end{array}$ & $\begin{array}{l}\mathrm{P}_{1} \\
(\text { days })\end{array}$ & $\mathrm{S}_{1}$ & $\begin{array}{l}\Delta \mathrm{t}_{\text {RISS }} \\
(\text { days })\end{array}$ & $\mathrm{m}_{\mathrm{RISS}}$ & $\begin{array}{l}\log \left[\tau_{c}\right] \\
(\mathrm{yr})\end{array}$ & $\begin{array}{l}\log [B] \\
(\mathrm{G})\end{array}$ \\
\hline $\mathrm{J} 0847-4316$ & 5.97 & 3.4 & 2438 & 69 & $1040_{-167}^{+912}$ & $89 \%$ & 118 & 0.11 & 5.9 & 13.4 \\
$\mathrm{~J} 1317-5759$ & 2.64 & 3.0 & 2324 & 94 & $560_{-55}^{+68}$ & $44 \%$ & 31 & 0.15 & 6.5 & 12.8 \\
$\mathrm{~J} 1444-6026$ & 4.76 & 5.5 & 2372 & 72 & $145 \pm 4$ & $5 \%$ & 197 & 0.09 & 6.6 & 13.0 \\
$\mathrm{~J} 1754-30$ & 1.32 & 2.2 & 2372 & 48 & $994_{-161}^{+239}$ & $95 \%$ & 21 & 0.17 & - & - \\
$\mathrm{J} 1819-1458$ & 4.26 & 3.6 & 2375 & 72 & $1260_{-240}^{+386}$ & $97 \%$ & 117 & 0.11 & 5.1 & 13.6 \\
$\mathrm{~J} 1826-1419$ & 0.77 & 3.2 & 2375 & 55 & $162 \pm 5$ & $24 \%$ & 65 & 0.13 & 6.1 & 12.4 \\
$\mathrm{~J} 1846-0257$ & 4.47 & 5.2 & 2193 & 46 & $135 \pm 4$ & $89 \%$ & 152 & 0.10 & 5.6 & 13.4 \\
$\mathrm{~J} 1913+1330$ & 0.92 & 5.7 & 1874 & 27 & $178 \pm 9$ & $48 \%$ & 87 & 0.13 & 6.2 & 12.4 \\
\hline
\end{tabular}




\subsubsection{Periodicity in pulse arrival times}

We have searched for periodicities in the pulse arrival times. In order to do this, we have created a time series by accounting for all rotations of the pulsar during each observation and assigning a delta function (i.e one for each detection and zero for each non-detection). We performed this search on the entire time span of observations.

In the time series for the entire time span of observations, we searched over $\sim 1,000,000$ periods spaced as outlined at the beginning of section 2.3.1, ranging from $\sim 25$ minutes to 2300 days. Six RRATs PSRs J0847-4316, J1317-5759, J1754-30, J1819-1458, J1826-1419, and J1913+1330, show significant periodicities in the arrival times on these timescales. The peaks of highest significance for PSRs J0847-4316, J1317-5759, J1754-30, J1826-1419, and J1913+1330 are at 3.8, 1.6, 1.4, 3.6 and 11.3 hours respectively, while PSR J1819-1458 shows a long term periodicity of 2102 days. There are many other significant periods and harmonics for these RRATs, with eight, three, one, thirteen, fifteen, and eight independent (i.e. non-harmonically related) periodicities with significance greater than $99 \%$ (2.5 $\sigma$ ) for PSRs J0847-4316, J1317-5759, J1754-30, J1819-1458, J1826-1419, and J1913+1330, respectively (See Table 2.2). These periodicities range from hours to years. Because of the large number of detected periodicities, we do not list them all here.

In order to determine the time dependence of the periodicities, we divided the time series to halves and quarters and performed the search again. For every RRAT, 
all periodicities detected in the full series were re-detected in at least one quarter subsection with lower significances. None of the periodicities were re-detected in every quarter, though seven out of the eight non-harmonically related periodicities of PSR J1913+1330 were re-detected in three of the quarter datasets. Similarly five out of the 13 non-harmonically related periodicities of PSR J1819-1458 were re-detected in three quarters with significances greater than $95 \%$. The rest were re-detected in only one or two quadrants.

All detectable (i.e. within the searched range) non-harmonically related periodicities of PSR J1913+1330 were re-detected in both halves of the dataset. For the rest of the RRATs only about five (for PSR J1819-1458) to two (for PSR J1913+1330) independent periodicities were re-detected in both halves. However every periodicity was re-detected in at least one half section of the dataset with lower significance. These results in general show that the periodicities persist throughout the entire time span of observations.

In order to gauge the reality of the periodicities, we randomized the time series of detections and non-detections by placing the pulses randomly within the observation windows and repeating the analysis. We found no periodicities with significance greater than $30 \%$ in any of these randomised time series, which suggests the periodicities found are real. Figure 2.1 shows the power spectra for the pulse arrival times from the randomized time series for the eight RRATs. 
Table 2.2: Periodicity in the arrival time $\mathrm{P}_{2}$, and the number of harmonics (nh) detected for each periodicity in the entire time series for the six RRATs. Only the most significant harmonic for each period with a significance larger than $99 \%$ is given. The periodicities are listed in the order of their spectral powers. We required the ratio between integer multiples of periods to be less than 1.001 to be considered a harmonic. The errors on the periodicities are calculated as half of the period bin size, which depends on the frequency resolution that depends on the number of frequencies searched and the timespan of observations. For small periods the error is the resolution of the periodogram and for larger periods such as 1, 10, 100 and 1000 days (for RRAT PSR J1819-1458) the errors are $10^{-6}, 10^{-3} 10^{-2}$, and 5 days respectively.

\begin{tabular}{|c|c|c|c|c|c|c|c|c|c|c|c|}
\hline \multicolumn{2}{|c|}{\begin{tabular}{l}
\multicolumn{2}{l}{ PSR J0847-4316 } \\
$\mathrm{P}_{2} \quad \mathrm{nh}$ \\
(days)
\end{tabular}} & $\begin{array}{l}\text { PSR . } \\
\mathrm{P}_{2} \\
\text { (days) }\end{array}$ & $\begin{array}{l}317-5759 \\
\text { nh }\end{array}$ & $\begin{array}{l}\text { PSR. } \\
\mathrm{P}_{2} \\
\text { (days) }\end{array}$ & $\begin{array}{l}754-30 \\
\mathrm{nh}\end{array}$ & $\begin{array}{l}\text { PSR } \\
\mathrm{P}_{2} \\
\text { (days }\end{array}$ & $\begin{array}{l}819-1458 \\
\text { nh }\end{array}$ & $\begin{array}{l}\text { PSR J1 } \\
\mathrm{P}_{2} \\
\text { (days) }\end{array}$ & $\begin{array}{l}6-1419 \\
\text { nh }\end{array}$ & \begin{tabular}{|l} 
PSR J1 \\
$\mathrm{P}_{2}$ \\
(days)
\end{tabular} & $\begin{array}{l}913+1330 \\
\text { nh }\end{array}$ \\
\hline $\begin{array}{l}0.16 \\
1.22 \\
265.7 \\
3.08 \\
0.753 \\
0.343 \\
0.998 \\
0.599\end{array}$ & $\begin{array}{l}10 \\
16 \\
1 \\
16 \\
1 \\
14 \\
16 \\
11\end{array}$ & $\begin{array}{l}0.079 \\
1.16 \\
0.138 \\
0.167\end{array}$ & $\begin{array}{l}7 \\
1 \\
6 \\
4\end{array}$ & 0.066 & 2 & $\begin{array}{l}2102 \\
0.996 \\
1187 \\
1.22 \\
0.907 \\
0.776 \\
0.548 \\
4.668 \\
0.153 \\
3.050 \\
19.03 \\
6.942 \\
10.20\end{array}$ & $\begin{array}{l}1 \\
33 \\
1 \\
34 \\
38 \\
39 \\
36 \\
26 \\
20 \\
13 \\
8 \\
9 \\
16\end{array}$ & $\begin{array}{l}0.158 \\
0.188 \\
0.869 \\
0.026 \\
0.036 \\
0.300 \\
1.462 \\
1.239 \\
0.048 \\
0.407 \\
10.934 \\
7.353 \\
162.89 \\
327.001 \\
117.841\end{array}$ & $\begin{array}{l}325 \\
311 \\
143 \\
969 \\
557 \\
255 \\
87 \\
100 \\
490 \\
213 \\
14 \\
20 \\
1 \\
1 \\
1\end{array}$ & \begin{tabular}{|l}
0.479 \\
7.601 \\
0.324 \\
1.624 \\
0.618 \\
34.657 \\
0.244 \\
1874
\end{tabular} & $\begin{array}{l}751 \\
124 \\
873 \\
437 \\
674 \\
28 \\
938 \\
2\end{array}$ \\
\hline
\end{tabular}




\subsubsection{Periodicities in daily pulse detection rates}

We have also applied this method to look for periodicities in the daily pulse detection rates. For each day, the observation length and the number of detected pulses were used to calculate the rate of pulse detection. Figure 2.2 shows how this rate varies for the eight RRATs. We then applied the Lomb-Scargle analysis to these rates, with the results of this analysis shown in Figure 2.3. We list the most significant period in Table 2.2 along with its significance.

We have performed white noise simulations and Monte Carlo simulations to verify the significance of the periodicities. In white noise simulations, the daily rates were replaced by random Gaussian noise. We could then calculate the power spectrum amplitude corresponding to the desired false alarm probability. In the second method Monte Carlo simulations were used to generate spectra from random time series which have the same sampling and the cumulative probability distribution of their maximum amplitude is calculated. We then fit this distribution to Equation 2.2, minimizing $\chi^{2}$ to determine an effective value for $N_{i}$ as this determines the false alarm probability for a given power spectral density. These tests verified the significances that we have quoted.

PSRs J1819-1458 and J1754-30 have periodicities with greater than $2 \sigma$ significance at 1260 and 994 days respectively, while PSRs J1846-0257 and J0847-4316 have periodicities with greater than $1 \sigma$ significance at 135 and 1040 days respectively. The remaining four RRATs do not show a periodicity of significance greater than the $1 \sigma$ level. The significances obtained for the peak power spectral density 
for PSRs J1819-1458 and J1754-30 from white noise simulations are 97\% and 96\% respectively. The significances calculated from Monte Carlo simulations are $97 \%$ and $89 \%$.

These periodicities are different from the ones detected in their pulse arrival times except for the $1260_{-240}^{+386}$ day periodicity of PSR J1819-1458, which is close to the pulse arrival time periodicity of $1186 \pm 7$ days. In general the pulse arrival time and pulse rate recurrence periodicities are expected to be independent though it is possible for them to be the same. In order to further gauge the reliability of our results for PSRs J1819-1458 and J1754-30, we created 1000 random sequences by assigning the measured pulse detection rates to randomly selected MJDs and calculated the number of times a peak of the same or higher significance appeared in the 1000 trials. This number was six and ten for PSRs J1819-1458 and J1754-30, respectively, suggesting that the periodicities detected are real and that their significances may in fact be underestimated. However, the detected periodicities are at $40 \%$ and $50 \%$ of the total observations lengths for PSRs J1754-30 and J1819-1458, respectively (see Table 2.2); longer observation spans are necessary to determine whether they are real. We also note that the significance of the peaks depends on the ranges of frequencies searched and that we have not searched frequencies higher than the Nyquist frequency or lower than $1 / T$.

In order to determine whether there was any dependence of significance on period, we simulated sinusoidal signals of various periods with additive random Gaussian noise and then applied the Lomb-Scargle algorithm. We found that the significance of the detected periodicities is independent of period. 


\subsubsection{Randomness tests on pulse arrival times}

The Kolmogorov-Smirnov (KS) test (see, e.g., Press et al. 1986) is a statistical procedure which determines the degree to which two datasets differ. It compares the cumulative probability distributions of both datasets by calculating the maximum deviation

$$
D=\max _{-\infty<x<\infty}\left|C_{1}(x)-C_{2}(x)\right|
$$

of cumulative distribution functions (CDFs) $C_{1}$ and $C_{2}$. The accuracy of this test increases with the number of data points and is expected to be accurate for four or more points (Stephens, 1970). From this the probability that two arrays of data values are drawn from the same distribution can be calculated. Small values of this probability (i.e. large values of $D$ ) suggest that the distributions being tested differ.

The test can therefore be used to explore whether the observed pulse sequences are consistent with random distributions. We have done this both by comparing the CDF of our data with the CDF of a simulated randomly distributed pulse sequence (i.e. kstwo test as implemented in Numerical Recipes) and by comparing the CDF of our data directly with the uniform CDF (i.e. ksone test). We have performed this test both for single days and for the entire time span of observations. For each single dataset, we created simulated distributions by placing the same number of detected pulses at random times within the observation, with the constraint of allowing only one detected pulse per rotation. We also tested randomness on longer time spans by creating random time sequences of length equal to the total observation lengths.

In Table 2.3, we list the total number of pulses observed from each RRAT along 
Table 2.3: Total observation time, total number of detected pulses, minimum, maximum, mean (with standard deviation in parentheses) of the number of pulses observed per day, average probability of short term randomness from the simulations $\left(\mathrm{P}_{\mathrm{r} 1}\right)$, the rms of the average probability $\left(\sigma_{1}\right)$, average probability of long term randomness from the simulations $\left(\mathrm{P}_{\mathrm{r} 2}\right)$, the rms of the average $\left(\sigma_{2}\right)$, average probability of short term randomness from direct comparison with the uniform $\operatorname{CDF}\left(\mathrm{P}_{\mathrm{u} 1}\right)$, and the average probability of long term randomness from direct comparison with the uniform CDF $\left(\mathrm{P}_{\mathrm{u} 2}\right)$. The probabilities for the simulations were calculated for 100 trials. The duration of each observation was 30 minutes to 1 hour on average; as can be seen from $\mathrm{N}_{2}$ there was one very long observation for PSR J1819-1458. The days with less than three pulses detected have not been included in $\mathrm{P}_{\mathrm{r} 1}$ or the $\mathrm{P}_{\mathrm{u} 1}$ probability calculation. The numbers outside and inside parentheses for $\mathrm{P}_{\mathrm{r} 1}, \sigma_{1}$ and $\mathrm{P}_{\mathrm{u} 1}$ indicate those for days with more than two/three pulses detected.

\begin{tabular}{llllllllllll}
\hline $\begin{array}{l}\text { Name } \\
\text { PSR }\end{array}$ & $\begin{array}{l}\mathrm{T} \\
(\mathrm{hr})\end{array}$ & $\mathrm{N}_{\mathrm{p}}$ & $\mathrm{N}_{1}$ & $\mathrm{~N}_{2}$ & $\mathrm{~N}_{\text {mean }}$ & $\mathrm{P}_{\mathrm{r} 1}$ & $\sigma_{1}$ & $\mathrm{P}_{\mathrm{r} 2}$ & $\sigma_{2}$ & $\mathrm{P}_{\mathrm{u} 1}$ & $\mathrm{P}_{\mathrm{u} 2}$ \\
\hline $\mathrm{J} 0847-4316$ & 49 & 141 & 0 & 11 & $2.04(2.6)$ & $0.52(0.53)$ & $0.15(0.13)$ & $5 \times 10^{-3}$ & 0.02 & $0.37(0.37)$ & $5 \times 10^{-8}$ \\
$\mathrm{~J} 1317-5759$ & 50 & 256 & 0 & 12 & $2.72(2.8)$ & $0.50(0.51)$ & $0.16(0.15)$ & $3 \times 10^{-3}$ & 0.01 & $0.42(0.46)$ & $1 \times 10^{-3}$ \\
$\mathrm{~J} 1444-6026$ & 85 & 41 & 0 & 4 & $0.57(1.0)$ & $0.47(0.45)$ & $0.11(0.11)$ & 0.54 & 0.29 & $0.32(0.23)$ & 0.31 \\
$\mathrm{~J} 1754-30$ & 60 & 40 & 0 & 3 & $0.83(0.9)$ & $0.09(-)$ & $2 \times 10^{-3}(-)$ & 0.03 & 0.07 & $3 \times 10^{-3}(-)$ & $1 \times 10^{-3}$ \\
$\mathrm{~J} 1819-1458$ & 45 & 1102 & 1 & 165 & $15.31(19.5)$ & $0.49(0.49)$ & $0.15(0.15)$ & $3 \times 10^{-5}$ & $2 \times 10^{-4}$ & $0.42(0.47)$ & $2 \times 10^{-12}$ \\
$\mathrm{~J} 1826-1419$ & 53 & 60 & 0 & 13 & $1.09(2.5)$ & $0.17(0.16)$ & $0.06(0.05)$ & $1 \times 10^{-4}$ & $4 \times 10^{-4}$ & $0.05(0.04)$ & $2 \times 10^{-3}$ \\
$\mathrm{~J} 1846-0257$ & 37 & 39 & 0 & 4 & $0.85(1.2)$ & $0.41(0.29)$ & $0.17(0.03)$ & 0.49 & 0.26 & $0.24(0.06)$ & 0.46 \\
$\mathrm{~J} 1913+1330$ & 19 & 138 & 0 & 27 & $5.11(7.8)$ & $0.18(0.17)$ & $0.13(0.14)$ & $6 \times 10^{-7}$ & $8 \times 10^{-6}$ & $0.06(0.07)$ & $2 \times 10^{-18}$ \\
\hline
\end{tabular}

with the numbers of pulses detected on individual days. We also list the probabilities of the pulse sequences being random on a single day, averaged over all days of observation by comparing with simulated random pulse sequences and directly with a uniform CDF. The simulated probabilities on each day are averages of 100,000 trials of different randomly generated datasets. We also list the rms deviations of the averages. Table 2.3 also lists the probability of pulses being randomly distributed on long data spans for simulated data (again averaged over 100,000 realizations) and through comparison with a uniform CDF. Figure 2.4 illustrates this comparison for two RRATs.

In general, the pulse distributions appear to be consistent with random distributions. For simulated data, the single-day pulse distributions have probabilities 

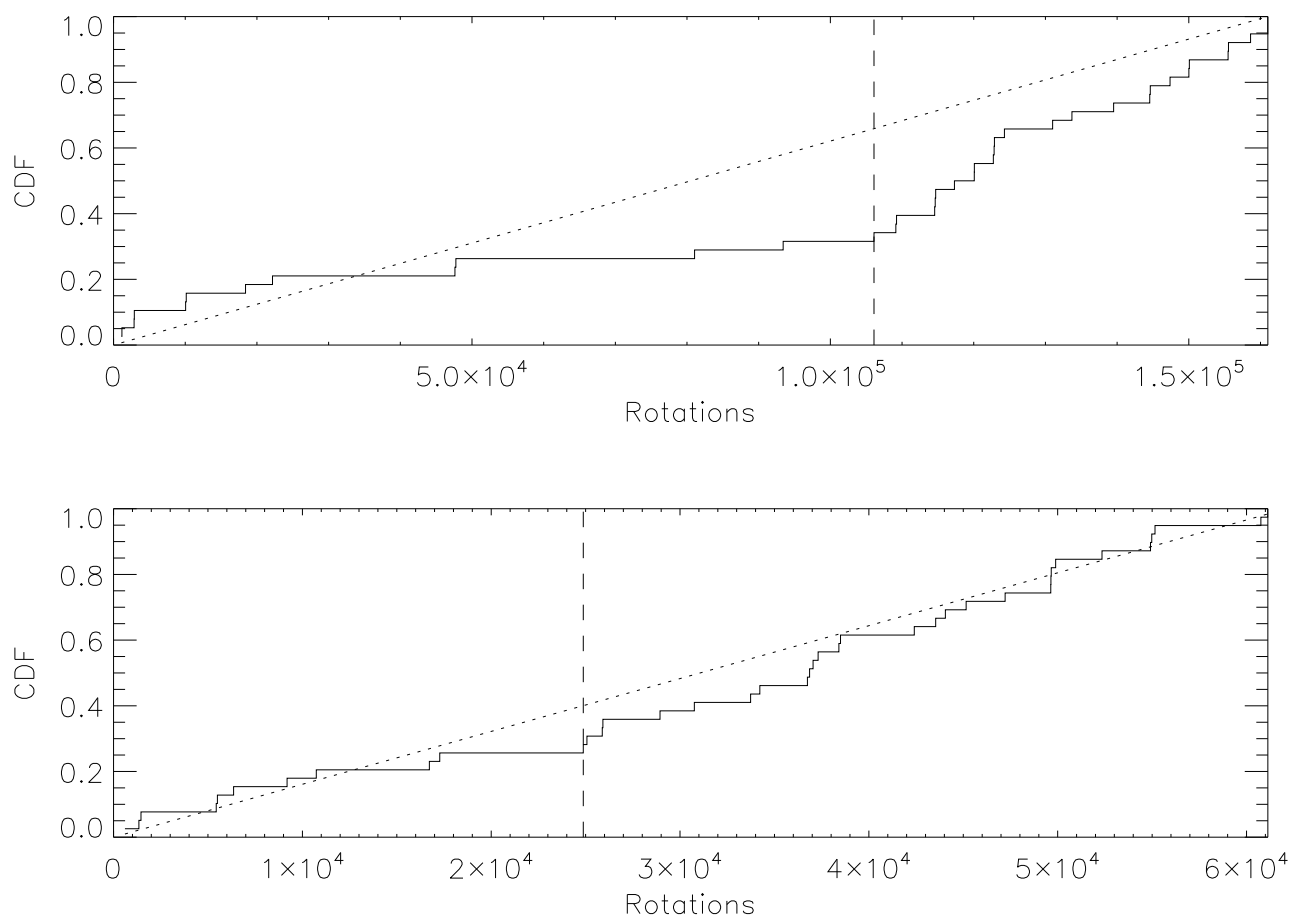

Figure 2.4: Cumulative distribution function for data over the entire time span of observations of PSR J1754-30 (an example of a non-random pulse sequence) and PSR J1444-6026 (an example of a random pulse sequence) compared with the CDF of a uniform distribution (dashed line). The figure also shows the point at which the KS statistic $D$ is measured (vertical dashed-dotted line). Small values of $D$ imply that the distributions are similar to each other. It can be seen that the first plot differs significantly from a uniform distribution, whereas the second does not. The probabilities of randomness are 0.001 and 0.31 for PSRs J1754-30 and J1444-6026 respectively. 
ranging from 0.09 (for PSR J1754-30) to 0.52 (for PSR J0847-4316) of being random when only days with more than two detections are considered. When days with more than three are considered the probabilities range from 0.16 (for PSR J1826-1419) to 0.53 (for PSR J0847-4316), indicating that the bias for small numbers of pulses is small (there are no epochs with three or more pulses for PSR J1754-30). When we compare directly with a uniform CDF, PSRs J1826-1419 and J1913+1330 show more evidence (i.e. probabilities of 0.05 and 0.06 ) for nonrandom pulse emission. PSR J1754-30 shows evidence for non-random behavior in both tests. As this is the RRAT with the lowest overall pulse rate, however, the tests are not very accurate.

The total number of detected pulses from each source ranges from $\sim 40$ to 1100, making our tests for randomness on long time scales very sensitive. When long time spans are considered, only PSRs J1444-6026 and J1846-0257 are consistent with random distributions. The probabilities given by using the ksone and kstwo tests are similar. This is not surprising given the large numbers of pulses. We have tested these analysis techniques on intervals between pulses and checked for their consistency with an exponential distribution. Both pulse arrival time and pulse interval tests give identical results as expected. Furthermore we have searched for any correlation between the pulse intervals on different timescales and have found none.

We explored the possibility of the telescope zenith angle at the time of the observation being responsible for variations in pulse detection rates, as the system temperature depends on this factor and to account for effects based on varying 
gain and spillover. The KS test was carried out on pulse sequences for lower and higher angles (i.e. angles less and greater than 45 degrees) for this purpose. These results indicate that there is little dependence of the detected rates on the zenith angle, with probabilities ranging from 0.31 (for PSR J1317-5759) to 0.49 (for PSR J1819-1458).

We have also searched for clustering of pulses. For all of the RRATs, the pulses usually occur singly with occasional consecutive pulses detected. We have measured the total number of doublets, triplets and quadruplets (i.e. instances of two, three, and four consecutive pulses) and compared with the number of doublets, triplets and quadruplets found in simulated random distributions. Table 2.4 lists the results of our analysis for 1000 simulated distributions. The number of doublets detected is higher than the number expected for a random distribution for both PSRs J1819-1458 and J1317-5759. PSR J1819-1458 shows many more doublets, triplets and quadruplets than expected, with one instance of nine consecutive pulses. The mean duration of the on states (at which pulses are detected) for the eight RRATs in units of the period are also listed in Table 2.4. These were calculated by taking into account the number of detected multiplets and their duration. For the RRATs which do not exhibit multiplets, we can only give an upper limit to the on-state duration of one period as the mean on-state will be less than one period. 
Table 2.4: Measured number $\mathrm{N}_{\mathrm{m}}$ of doublets, triplets, quadruplets vs. the expected number $\mathrm{N}_{\mathrm{e}}$, probability of occurrences under an assumed Poisson distribution $\mathrm{P}_{\mathrm{p}}$ for eight RRATs and the mean duration of the on state in units of the period. The expected numbers are the means of 1000 trials of randomly generated fake distributions.

\begin{tabular}{lllllllllll}
\hline \multirow{2}{*}{$\begin{array}{l}\text { Name } \\
\text { PSR }\end{array}$} & \multicolumn{3}{c}{ Doublets } & \multicolumn{4}{c}{ Triplets } & \multicolumn{2}{c}{ Quadruplets } & \multicolumn{2}{l}{ On state duration } \\
(upper limit)
\end{tabular}

\subsection{Discussion}

We have shown that six of the RRATs have periodicities of significance greater than $99 \%$ in the pulse arrival times. The periods of the most significant peak range from 1.4 hours (for PSR J1754-30) to 2102 days (for PSR J1819-1458). No significant periodicities were detected upon randomizing the time series, showing that these periodicities are real. We do not find any relationship between the number and significance of detected periodicities and spin-down properties such as period or characteristic age. It is possible that some of the periodic behavior is due to refractive scintillation. However, the number and wide range of timescales of the periodicities are impossible to explain with refractive scintillation alone.

The shorter timescale periodicities in pulse arrival times are similar to typically observed nulling timescales, which range from minutes to days (Wang et al., 2007). Explanations for pulsar nulling include an empty sight-line passing through the subbeam structure (Deshpande \& Rankin, 2001), a reversal of the emission direction 
(Melikidze \& Gil, 2006), pulsar emission ceasing temporarily due to intermittent failure of pair production (Zhang et al. 2007), an asteroid belt of material (Cordes \& Shannon, 2008) or changes in magnetospheric currents (Lyne et al., 2010; Wang et al., 2007). Any combination of these could explain the extreme pulse-to-pulse variability of the RRATs and also the longer term periodicities.

The significant periodicities found in the RRAT pulse arrival times may suggest a relationship with pulsars whose spin-down rates and pulse shapes undergo periodic variations, with implied changes in magnetospheric particle density (Lyne et al., 2010). The prototype of this source class is B1931+24 (Kramer et al. 2006). The asteroid belt model of (Cordes \& Shannon, 2008) attributes the 40-day on/off timescale of this pulsar to an asteroid with eccentric 40-day orbit. It may be that the pulse-to-pulse variability of the RRATs is due to a similar process happening on very short timescales. However, because the on states of the RRATs are so short, it is impossible to measure period derivatives during the on and off states. It could also be that a similar process is causing the longer term periodicities in pulse arrival times.

If we apply the model of (Cordes \& Shannon, 2008) to the RRATs, multiple asteroids of an asteroid belt could be responsible for the observed periodicities in the arrival times. This is consistent with the large root mean square timing residuals which range from $1.1 \mathrm{~ms}$ (for PSR J1913+1330) to $11.2 \mathrm{~ms}$ (for PSR J0847-4316) of these RRATs as an earth-sized asteroid would induce residuals of the order of $1 \mathrm{~ms}$ (Cordes \& Shannon, 2008). However it is possible that much of these large residuals are due to pulse-to-pulse jitter, indicating that the true asteroid mass cannot be 
determined by the residuals only.

The periodic fluctuations in pulse arrival times could also be due to nonradial oscillations which drive different emission modes, as often seen in white dwarf stars (Rosen et al., 2011). The fundamental oscillation periods for neutron stars are expected to be on the order of milliseconds (Reisenegger \& Goldreich, 1992) to seconds (McDermott et al., 1988) for g-modes. These are far too short to explain the multiple periodicities seen, but it is possible that we are observing the beat frequency between a non-radial oscillation period and that from a longer timescale process like those observed in Lyne et al. (2010).

We also searched for periodicities in the daily pulse detection rates. Six of the RRATs do not show any significant periodicities in their daily pulse detection rates over timescales of months to years. The exceptions are PSR J1819-1458, which exhibits a 1260 day period with a significance of 97\%, and PSR J1754-30, which exibits a 994 day period with significance of $95 \%$. We detect a peak of higher significance only $0.6 \%$ and $1.0 \%$ of the time in 1000 trials in which the rates were randomly assigned to the MJDs for PSRs J1819-1458 and J1754-30 respectively, suggesting that the significance of the peak may be underestimated by the LombScargle algorithm. Given eight trials, if all of the RRATs had no periodicities, we would expect to find one periodicity with significance greater than $88 \%$. However, two RRATs with significances greater than $95 \%$ are not expected. The predicted timescales for refractive interstellar scintillation of 117 days for PSR J1819-1458 and 21 days for PSR J1754-30 are much smaller than the reported periodicities in pulse detection rates, indicating that these periodicities are not likely due to scintillation. 
However, these periodicities are roughly half of the total data span. Therefore, a longer time span of observations is necessary to confirm them as significant.

There is evidence for changes in period derivative associated with changes in pulse detection rate for PSR J1819-1458 (Lyne et al., 2009). The peak in the rate immediately following the first (and largest) glitch at MJD 53926 (Lyne et al., 2009) hints at a correlation between glitches and emission properties. If confirmed in future glitches, this correlation might suggest a link with the magnetars, for which radiative changes often accompany glitches (e.g. Dib et al., 2008b) or the class of mode changing pulsars whose period derivative undergoes quasi-periodic changes (Lyne et al., 2010).

All of the sources exhibit random pulse distributions on single days. This is similar to that observed for giant pulses (Knight et al., 2006). However, the pulses from the RRATs are wider than those of observed giant pulses and the pulse amplitude distributions are different (McLaughlin et al., 2009; Miller et al., 2010). This therefore supports the idea of Weltrevrede et al. (2006), who suggest that the RRATs could be normal, but more distant, pulsars like B0656+14 which emits very bright, narrow pulses and a distribution of weaker, broader pulses.

Over longer time spans, the pulse sequences of six of the RRATs show evidence for non-random behaviour. These same RRATs show significant periodicities in arrival times. The external influence from, e.g., an asteroid belt (Cordes \& Shannon, 2008) could explain this through uneven distributions of material. However, it is difficult to relate this long term non-randomness to either normal or nulling pulsars as, to our knowledge, this has not been explored for either of these source classes. We 
do not find any obvious relationship between the randomness and derived spin-down properties such as age, period, and surface dipole magnetic field.

Nulling pulsars tend to have on-off states which persist over more than one pulse period (Wang et al., 2007) whereas the large majority of the RRAT pulses occur singly, aside from a few pulses from PSRs J1819-1458 and J1317-5759. Redman \& Rankin (2009) showed that the majority of pulsars null non-randomly and several studies (e.g. Rankin \& Wright 2008 and Herfindel \& Rankin 2009) have revealed periodicities in the null cycles for some pulsars. The RRATs have quite different properties in these respects, but because the nulls of some pulsars (e.g. B0834+06, B1612+07, and B2315+21; Redman \& Rankin (2009)) are random and single-pulse nulls are occasionally observed, we cannot exclude the possibility of the RRATs being an extreme case of nulling. This is supported by the similar ages and spin periods of many RRATs and nulling pulsars and the strong correlation between nulling and pulsar age (Wang et al., 2007).

In summary, we detect highly significant periodicities in the arrival times of six of the RRATs. We detect no highly significant periodicities in the long-term pulse detection rates for the RRATs, although we find tentative periodicities for PSRs J1819-1458 and J1754-30. All of the RRATs show random behavior on a single day and most of the RRATs show non-random behavior on long timescales. Most of the RRATs emit pulses singly, but a few do show evidence for clustering of pulses. It is clear that there are periodicities in the pulse arrival times for these objects. The cause of these could be circumstellar material, non-radial oscillations, or another process. While we cannot completely eliminate any of the ideas, our 
results favor the asteroid belt model and are consistent with extreme nulling pulsars or distant pulsars emitting detectable bright pulses and non-detectable weak pulses. Radio monitoring over longer time spans and observations at other wavelengths may be useful to further understand the reasons for the RRATs' unusual emission. More theoretical work and further studies of the randomness of pulse emission in normal pulsars with different properties is also necessary. 


\section{Chapter 3}

\section{Interstellar Time Delay Correction}

\section{Submitted to ApJ, with co-authors D. R. Stinebring, M. A. McLaughlin, P. B. Demorest and G. Jones}

\subsection{Introduction}

Gravitational waves (GWs) are a key prediction of Einstein's theory of general relativity and their existence has been supported through timing measurements of the orbital decay of the Hulse-Taylor binary system B1913+16 (Harrison \& Tademaru, 1975). Many experiments aim to detect these waves directly through the measurement of light travel changes between objects. Complementary to interferometerbased GW detection experiments like LIGO, pulsar timing is sensitive to nanohertz frequency GWs. The change in light travel time between the earth and a pulsar due to a passing GW results in a delay in the time of arrival (TOA) of pulses (Detweiler, 1979). Given a timing model that accounts for parameters such as pulsar period, period derivative, position, proper motion and other orbital parameters, we calculate residuals, or the differences between measured and model TOAs.

These residuals will contain the signatures of gravitational waves. A stochastic background of GWs can be detected through searching for a correlation with angular separation in the timing residuals of an array of pulsars (Hellings \& Downs, 
1983). In order to detect the background due to supermassive black hole binaries, over 100 MSPs with root-mean-square (RMS) timing residuals of less than $100 \mathrm{~ns}$ are likely required (Jenet et al., 2005; Cordes \& Shannon, 2012). Currently over 40 millisecond pulsars are being timed by the North American Nanohertz Observatory for Gravitational waves (NANOGrav), with RMS timing residuals of nearly all pulsars at the sub-microsecond level (Demorest et al., 2013; McLaughlin, 2013). In addition to GWs, other effects such as interstellar medium (ISM) propagation and rotational irregularities will affect the arrival times of pulses. Fortunately, ISM effects are chromatic and therefore multi-frequency observations can be used to at least partially correct for these variations.

Electromagnetic radiation from pulsars is delayed as it travels through the ionized plasma of the ISM. The three prominent known effects are (1) dispersion caused by the change in radio wave speed due to refraction, (2) scattering and scintillation due to inhomogeneities in the medium (Rickett, 1969) which results in a random interference pattern on the observer plane, and (3) Faraday rotation which is rotation of the plane of linear polarization due to a magnetized plasma. See Chapter 1 for more information. All timing algorithms correct for time-variable dispersion to high accuracies (Keith et al., 2013). Faraday rotation could split the profile due to delays between the left and right circularly polarized waves (Cordes \& Shannon, 2010) but the TOA errors from this effect are negligible. We do not expect Faraday rotation to result in TOA fluctuations if polarization calibration is done correctly. In this paper we concentrate on removal of scattering effects, which are more difficult to correct but can cause sizeable fluctuations in TOAs. 
The scattering process delays the pulse TOA due to refraction and multipath propagation. While the most prominent scattering effect is pulse broadening due to multipath propagation, other effects such as angle of arrival variations also contribute to the pulse delay. Scintillation causes the pulse to appear brighter at certain times and frequencies, with characteristic scales determined by the distance to the pulsar, its velocity, the properties of the ISM along the line of sight, and the observing frequency; for a review of effects see Stinebring (2013). Figure 3.1 shows a schematic picture of how these various delays affect the signal. The long-term goal of pulsar timing is to correct for delays due to other intrinsic and extrinsic effects such that only the GW signature remains in the residuals.

ISM delays are observing frequency $(\nu)$ dependent, with dispersion and pulse broadening scaling as $\nu^{-2}$ and $\nu^{x}$, respectively, with $x \approx-4$ (Lorimer \& Kramer, 2005). Therefore pulse broadening, which is indicative of large amounts of scattering, is most prominent at low frequencies. In addition to the frequency dependence, pulse broadening has been empirically determined to have a roughly $\mathrm{DM}^{2}$ dependence (Bhat et al., 2004), where DM is the dispersion measure, or the integral of the electron density along the line of sight. Scattering delays are also expected to vary significantly with time due to the relative motion of the pulsar and the Earth changing the line-of-sight path through the ionized ISM. Hemberger \& Stinebring (2008) used secondary spectra of pulsar B1737+13 to measure scattering delays between 0.2 and $2.2 \mu$ s over $\sim 270$ days of observation at a radio frequency of 1400 MHz. Ramachandran et al. (2006) showed that scattering delays vary between $\sim 100$ and $\sim 140 \mu$ s over $\sim 10$ years for B1937+21 at $327 \mathrm{MHz}$. 
Correcting for ISM scattering delays may be important for detecting GW signatures in our data (e.g., Foster \& Cordes, 1990). In addition, the average spectral index of millisecond pulsars is $\sim-1.4$ (Bates et al., 2013), meaning that these objects are $\sim 8$ times brighter at $430 \mathrm{MHz}$ than at $1400 \mathrm{MHz}$. The MSPs used in current timing experiments are selected to be nearby (i.e. <few kpc) and are generally timed at high frequencies $(\geq 800 \mathrm{MHz})$ in order to mitigate these dispersion and scattering effects. The ability to correct for the effects of scattering could improve timing at lower frequencies, resulting in increased signal-to-noise ratio $(\mathrm{S} / \mathrm{N})$. Finally, understanding the scattering phenomenon will lead to better quantifying the Galactic models for free electron density (Cordes \& Lazio, 2002) and the distribution of scattering material along the line of sight (Cordes \& Rickett, 1998).

Several methods have been proposed to estimate scattering timescales. These methods assume that the ISM acts as a linear filter with a voltage pulse broadening function $(\mathrm{PBF})$, also referred to as the impulse response function, which is convolved with the intrinsic pulsar signal to produce the observed pulse. For scattering by a single thin screen with a square law structure function (Cordes \& Rickett, 1998), the ensemble-averaged PBF is a one-sided exponential. Kuz'min \& Izvekova (1993) showed that a descattered pulse can be restored by fitting the observed profile to a Gaussian convolved with a one-sided exponential function. However, these methods usually require assumptions about the functional form of the PBF, which is dependent on the spatial distribution and inhomogeneity spectrum of the scattering medium (Cordes \& Rickett, 1998). The scattering times can also be estimated from the auto correlation function $(\mathrm{ACF})$ of the pulsar dynamic spectra or from the 
cumulative delay function from pulsar secondary spectra (see, e.g., Hemberger \& Stinebring, 2008). However, these methods are limited by large uncertainties and a finite number of scintles, which are maxima in the interference pattern, within the observing bandwidth and observation time. Another method, which is based on a CLEAN algorithm, tests various PBF types to get the best fit, and requires assumptions about the PBF form (Bhat et al., 2003). This method can estimate PBFs when scattering delays are large and cause recognizable changes to pulse shapes, but is not optimal in the case of small delays. More recently Coles et al. (2010) showed that scattering is anti-correlated with pulse power and the TOA fluctuations can be reduced by $\sim 25 \%$ by removing those correlated components. Unlike these methods, cyclic spectroscopy (CS) directly accounts for phase changes of the electric field, thereby allowing a more accurate description of ISM effects.

The phase of the ISM transfer function, which is the frequency-domain representation of the $\mathrm{PBF}$, contains information about pulse broadening due to ISM delays. Recovering the phase information of the electric field to reconstruct the PBF has been successfully applied to pulsar dynamic spectra (Walker et al., 2008). In this paper we explore a phase retrieval method using the technique of CS, introduced in Demorest (2011) and further developed in Walker et al. (2013, hereafter WDS13). This method allows determination of the phase of a periodic signal, which can then be used to calculate scattering delays. This paper is a step in an ongoing analysis of the efficiency of CS for scattering delay correction. By means of a simulation that includes a realistic signal model, we show that CS can be used to accurately reconstruct the $\mathrm{PBF}$ for an achievable signal-to-noise ratio. Instead of simply a 


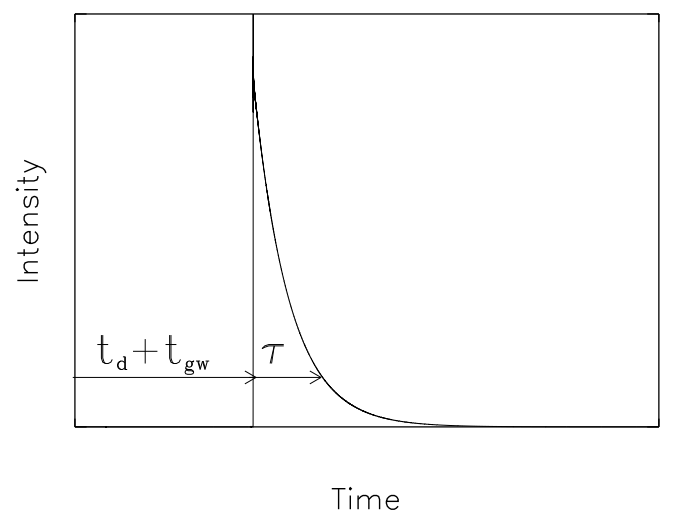

Figure 3.1: A simplified model that describes how some of the most prominent effects cause delays in the pulsar signal. Delays from gravitational waves, dispersion, and scattering of the intensity PBF are given by $t_{g w}, t_{d}$ and $\tau$, respectively. The former two effects cause time delays of the pulse while scattering also changes its shape, broadening it and thereby causing an additional delay.

TOA, this analysis supplies a TOA and a PBF, which is valuable additional information when used in a multi-frequency timing and analysis program. We introduce the theoretical formulation in Section 3.2; in Section 3.3 we present the details and results of our simulation; and in Section 3.4 we discuss future applications and the advantages of using CS over other methods.

\subsection{Theoretical background}

We begin by expressing the electric field vector as a function of time $t$ and position $\mathbf{r}$, as $\mathbf{E}(\mathbf{r}, t)=\mathbf{E}_{\mathbf{0}} \exp [-i(\mathbf{k} \cdot \mathbf{r}-\omega t)]$, where $\mathbf{k}$ and $\omega$ are the wave vector and angular frequency of the wave respectively. For a wave with frequency $f$, traveling in a medium with refractive index $\mu$ at a speed $c$, the wave vector is $k=(2 \pi / c) \mu f$. Frequency-dependent refractive index fluctuations in the medium cause a change in $k$, which corresponds to a change in the phase of the wave. Upon encountering a 
scattering region, in this case a thin screen of thickness $a$, the phase of the wave changes by an amount $\Delta \Phi=\Delta k a$ (see, e.g., Lorimer \& Kramer, 2005, for details). For a wave propagating in the $\hat{\mathbf{z}}$ direction, the phase of the wavefront becomes a function of $\mathrm{x}$ and $\mathrm{y}$ after passing through the phase screen. The phase changes will vary randomly along the wavefront, and hence the final phase changes will be randomly distributed. The final phase 'corrugation', causes angular broadening of the propagating radiation. Additionally, electron density variations that are large compared to the broadened "image" of the pulsar projected on the scattering screen will result in refractive effects. (See Rickett, 1990) for a review. The bent wavefront arrives later than the unscattered one, resulting in a scattering response that approximates an exponentially-decaying function in long-term averages (Williamson, 1972).

In general, pulsar (E-field) signals can be considered to be amplitude-modulated complex Gaussian noise (Rickett, 1975) so that

$$
x(t)=n_{1}(t) p(t)
$$

where $p(t)$ is a real-valued, positive definite pulse modulation function and $n_{1}(t)$ is complex Gaussian white noise.

Under the assumption that the interstellar medium affects only the phase of the propagating signal and not its amplitude, it acts as a linear filter with a voltage PBF $h(t)$ and a corresponding transfer function $H(\nu)$, where $h(t)$ and $H(\nu)$ are a Fourier transform pair. For an intrinsic pulsar voltage (E-field) signal $x(t)$, the 
observed voltage signal in the time domain will be $y(t)=x(t) * h(t)$, where $*$ denotes a convolution. This voltage signal $y(t)$ plus additive noise, discussed further below, is recorded by baseband observing systems. The frequency-domain representation of the signal will be $Y(\nu)=X(\nu) H(\nu)$, where $x(t)$ and $X(\nu)$ are a Fourier transform pair.

Following the notation of Demorest (2011), the "cyclic spectrum" as a function of radio frequency $\nu$ and cycle frequency $\alpha$ is then given by (Antoni, 2007)

$$
S_{y}(\nu ; \alpha)=E\left\{Y\left(\nu+\frac{\alpha}{2}\right) Y^{*}\left(\nu-\frac{\alpha}{2}\right)\right\}
$$

where $Y^{*}(\nu)$ is the complex conjugate of $Y(\nu)$ and $E$ represents the expectation value. For a true cyclostationary signal to have non-zero $S_{y}(\nu ; \alpha)$, the cyclic frequency $\alpha$ must take on discrete values such that $\alpha_{n}=n / P$, where $P$ is the pulse period. The cyclic spectrum can be further expanded as

$$
\begin{aligned}
S_{y}\left(\nu ; \alpha_{n}\right)=E\left\{X\left(\nu+\alpha_{n} / 2\right) X^{*}\left(\nu-\alpha_{n} / 2\right)\right\} & \times \\
& H\left(\nu+\alpha_{n} / 2\right) H^{*}\left(\nu-\alpha_{n} / 2\right) .
\end{aligned}
$$

Assuming that the variance of pulse-to-pulse noise $n_{1}(t)$ over a narrow band is $\sigma_{p}^{2}$ and $c_{n}$ is the $n^{\text {th }}$ complex Fourier coefficient of the Fourier transform of intensity modulation function $p_{I}(t)$, where $p_{I}(t)=p(t)^{2}$, we can express the spectrum of the 
intrinsic signal as

$$
E\left\{X\left(\nu+\alpha_{n} / 2\right) X^{*}\left(\nu-\alpha_{n} / 2\right)\right\}=c_{n} \sigma_{p}^{2}
$$

By invoking the fact that any complex function can be represented with amplitude $A$ and phase $\Phi$ as $A \exp [i \Phi]$, the phase of the cyclic spectrum $\Phi_{S_{y}}$, in terms of the phase of the transfer function $\Phi_{H}(\nu)$ and the phase of the Fourier coefficient $\Phi_{c_{n}}$, is

$$
\Phi_{S_{y}}(\nu ; \alpha)=\Phi_{H\left(\nu+\frac{\alpha_{n}}{2}\right)}+\Phi_{H^{*}\left(\nu-\frac{\alpha_{n}}{2}\right)}+\Phi_{c_{n}} .
$$

Since the cyclic spectrum is the quantity which can be calculated for any known observed voltage signal, and we are interested in reconstructing the transfer function, we need to express its phase in terms of the known quantities. We can write

$$
\Phi_{S_{y}}(\nu)-\Phi_{c_{n}}=\Phi_{H\left(\nu+\frac{\alpha_{n}}{2}\right)}-\Phi_{H\left(\nu-\frac{\alpha_{n}}{2}\right)} \approx \frac{d \Phi_{H}(\nu)}{d \nu} \alpha_{n} .
$$

Therefore the phase of the transfer function can be expressed in terms of the phase of the cyclic spectrum as

$$
\Phi_{H}(\nu) \approx \frac{1}{\alpha_{n}} \int_{-B / 2}^{\nu}\left(\Phi_{S_{y}}\left(\nu^{\prime}\right)-\Phi_{c_{n}}\right) \mathrm{d} \nu^{\prime},
$$

for $-B / 2 \leq \nu \leq B / 2$, where $B$ is the full bandwidth. Following Equation 3.2, we 
derive an expression for the amplitude of the cyclic spectrum $A_{S_{y}}$ as

$$
A_{S_{y}\left(\nu ; \alpha_{n}\right)}=c_{n} \sigma_{p}^{2} A_{H\left(\nu+\frac{\alpha_{n}}{2}\right)} A_{H^{*}\left(\nu-\frac{\alpha_{n}}{2}\right)},
$$

For the very small values of $\alpha_{n} \approx 10^{-4} \mathrm{MHz}$ for MSPs, we can assume $A_{H\left(\nu+\frac{\alpha_{n}}{2}\right)} \simeq$ $A_{H^{*}\left(\nu-\frac{\alpha_{n}}{2}\right)}$. Then the amplitude of the transfer function can be estimated to be

$$
A_{H} \simeq \sqrt{\frac{A_{S_{y}}}{\left|c_{n}\right| \sigma_{p}^{2}}}
$$

Once the phase of the transfer function is computed using the phase integral method described in Equation 3.7, and the amplitude of the cyclic spectrum is obtained from Equation 3.9, a complete reconstruction of the frequency-domain transfer function is possible. This can be transformed to the time domain to obtain the PBF.

There are multiple approaches to retrieving PBFs from cyclic spectra. Demorest (2011) and WDS13 recover the PBF from the cyclic spectra via a least square minimization of the difference between the modeled and actual cyclic spectrum. The second method, used in this analysis, is based on computing the phase of the transfer function directly via the phase integral method. We refer to this as the direct phase integration (DPI) approach and use it here for the sake of simplicity, as no iterative fitting is needed to arrive at the recovered PBF. The WDS13 approach includes an initialization step that is quite similar in character to the DPI method. We anticipate that when this technique is incorporated in timing analysis pipelines, the WDS algorithm (Walker et al., 2013), or future variants, will be used since the 
fitting technique will result in a more accurate $\mathrm{PBF}$ reconstruction for lower $\mathrm{S} / \mathrm{N}$ data.

\subsection{Simulations}

We start by forming a pulsar signal $x(t)=n_{1}(t) p(t)$, as outlined in Equation 3.1. For simplicity, we choose $p(t)=\exp \left[-(t / W)^{2}\right]$, a Gaussian-shaped modulation function whose width is $W$, and where $t$ spans the pulse period for a single pulse, and repeats itself to infinity. Variations in the single pulses are caused by varying $n_{1}(t)$ values.

For a scattering medium approximated by a single thin screen, and assuming that the refractive index fluctuations within the screen have a square-law structure function (e.g., Cordes \& Rickett, 1998), the voltage PBF has a one-sided exponential envelope (Cordes, 1976) and takes the form

$$
h(t)=n_{2}(t) U\left(t-t_{0}\right) \exp \left[-\frac{t-t_{0}}{\tau^{\prime}}\right]
$$

where $n_{2}(t)$ is complex Gaussian white noise, $U(t)$ is the unit step function, $t_{0}$ is any uncorrected time delay (relative to a fiducial pulse template), and $\tau^{\prime}=2 \tau$, where $\tau$ is the characteristic width in the intensity $\mathrm{PBF}$, which will be referred to as the scattering timescale hereafter. The inclusion of $n_{2}(t)$ in Equation 3.10 allows each run of the simulation to be an example of a "snapshot image" of the ISM (Goodman \& Narayan, 1989) for each realization of $n_{2}(t)$ and incorporates the effect of scintillation. We form the Fourier transform of the observed voltage signal 
$y(t)$ using the above transfer function

$$
Y(\nu)=X(\nu) H(\nu)+N_{\text {sys }}(\nu)
$$

where $N_{\text {sys }}$ is complex additive instrumental noise.

We simulate a pulsar with a period $P$, a pulse width $W$, where $P \gg W$, and a scattering timescale $\tau$, where $\tau<W$. For specificity, we consider a period of 1.6 ms, pulse width of $40 \mu \mathrm{s}$ in the intensity profile, and a mean scattering timescale of $5 \mu \mathrm{s}$ in the intensity $\mathrm{PBF} h_{I}$, where $h_{I}=|h(t)|^{2}$. These quantities are similar to the values for the bright MSP, B1937+21, observed at a frequency of $1 \mathrm{GHz}$. Amplitude modulated noise was produced in the frequency-domain as Gaussian white noise with variance $\sigma_{p}^{2}=1$, with the middle half of the spectrum removed, to allow for oversampling by a factor of two. The noise was then Fourier-transformed so it would be correlated in the time domain. This was then multiplied with the Gaussian pulse modulation function $p(t)=\exp \left[-(t / W)^{2}\right]$, as indicated in Equation 3.1, to produce a single pulse profile as emitted at the pulsar. The frequency-domain representation of the observed single pulse profile is then calculated using Equation 3.11, with the simulated ISM transfer function $H(\nu)$, which is the Fourier transform of $h(t)$ as given in Equation 3.11. We have included Gaussian white instrumental noise with variance $\sigma_{n}^{2}=1$ to each pulse and have oversampled the data by a factor of two in order to satisfy the requirements for calculating the cyclic spectrum (Antoni, 2007).

These simulated single pulses are used to compute the cyclic spectra via the frequency-domain approach, as outlined in Equation 3.2. For this purpose, cyclic 
spectra of different $\alpha_{n}$ cycle frequencies have been combined optimally, assuming that the phase of the cyclic spectrum is linearly proportional to the harmonic number $n$. Thus the weighted average of the cyclic spectrum can be expressed as

$$
S_{y}(\nu)=\frac{\sum_{n=1}^{n_{\max }} w_{n} A_{n}(\nu) e^{i \Phi_{n}(\nu) / n}}{\sum_{n=1}^{n_{\max }} w_{n}}
$$

where $A_{n}(\nu)$ is the amplitude and $\Phi_{n}(\nu)$ is the phase after the profile contribution has been subtracted off, as shown in the left hand side of Equation 3.6. Both $A_{n}(\nu)$ and $\Phi_{n}(\nu)$ correspond to the $n^{\text {th }}$ harmonic of the cyclic spectrum. The weight $w_{n}$ of a scattering time measurement $\tau_{n}$ from the cyclic spectrum of the $n^{\text {th }}$ cycle frequency is defined as

$$
w_{n}=\frac{1}{\delta \tau_{n}^{2}}
$$

Here, the error of the delay measurement $\delta \tau_{n} \propto\left|c_{0}\right| /\left(n\left|c_{n}\right|\right)$ since theoretically, the $\mathrm{S} / \mathrm{N} \propto\left|n c_{n}\right|$ where $c_{n}$ denotes the $n^{\text {th }}$ Fourier coefficient of the pulse modulation function, $p(t)$ (Appendix A). We have used $n_{\max }=5$ for this analysis. Then we use Equations 3.7 and 3.9 to recover the phase and amplitude of the transfer function, which is inverse-Fourier-transformed to the time-domain to get the voltage PBF. The pulse profiles and cyclic spectra were obtained from averaging $N_{p}=10^{4}$ single pulses, using a bandwidth of $10 \mathrm{MHz}$. Figure 3.3 shows one of these simulated average profiles with a scattering tail of $5 \mu \mathrm{s}$. The amplitude and phase of simulated 


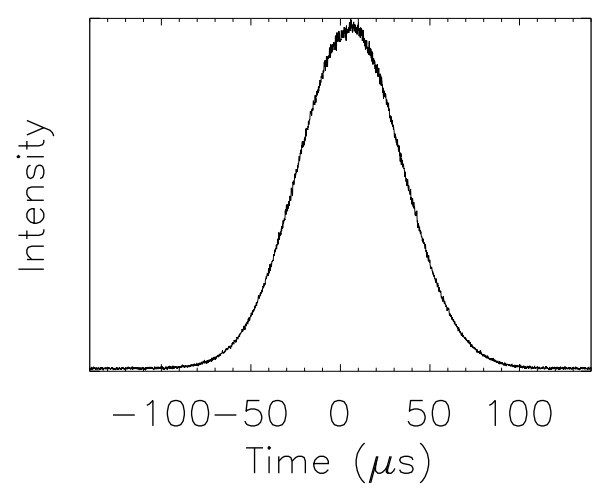

Figure 3.2: An example scatter-broadened average pulse profile (averaged over 10,000 pulses) as a function of pulse phase. The simulated pulsar has a period of $1.6 \mathrm{~ms}$ and a pulse width of $40 \mu \mathrm{s}$. The scattering timescale for this case is $5 \mu \mathrm{s}$.

cyclic spectra as functions of frequency are shown in Figure 3.3. Figure 3.4 shows the reconstructed intensity PBF.

\subsubsection{Scatter correction}

The scattering process broadens the pulse and delays the TOA by shifting its centroid (see, e.g., Coles et al., 2010). In practice, there will be other chromatic and achromatic delays in the data, such as those due to refraction, parallax, proper motion and GW signals, in addition to scattering, which will give a non-zero $t_{0}$ value in the PBF outlined in Equation 3.10. Therefore, $t_{0}$ can be considered as the non-scattered TOA, with all interesting time signatures remaining intact. In order to calculate $t_{0}$, we fit a one-sided exponential function to the recovered PBF in order to locate the rising edge of the PBF and use these $t_{0}$ values as our scatter-corrected TOAs.

The importance of using the location of the rising edge of the PBF as the 


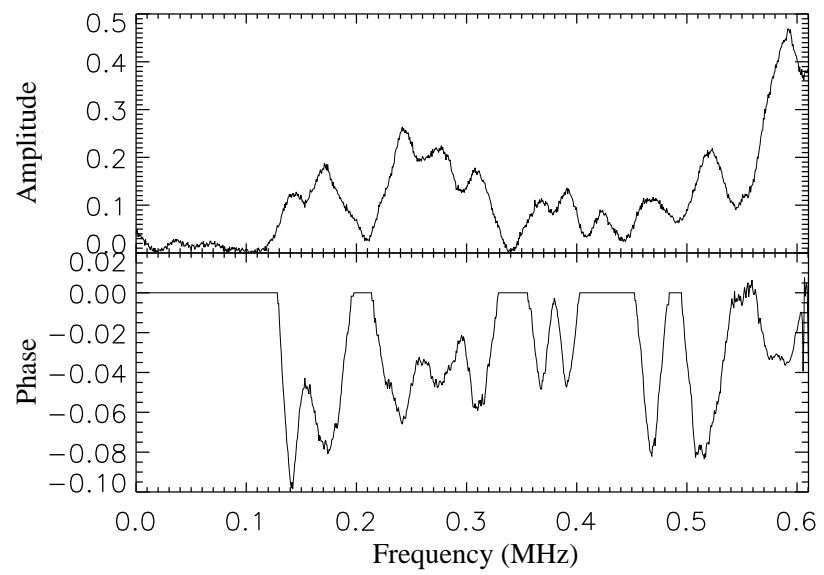

Figure 3.3: The amplitude (top) and phase (bottom) of a cyclic spectrum corresponding to the simulation parameters in Figure 3.3, as a function of frequency, for the first cycle frequency $(n=1)$. The phase has been set to zero for very small amplitudes in the cyclic spectrum and smoothed with a boxcar function for clarity. The noise is self-noise from the pulsar amplitude-modulated noise process after an integration of $N=10^{4}$ pulses.

scatter corrected TOA is that it allows us to retain other delays affecting the signal.

It is necessary to be able to correct for scattering without removing other effects such as parallax, proper motion, refractive effects and most importantly the delays from GWs. Even though calculating the centroid of the PBF gives a good estimate of all the delays involved, using the centroid to correct for scattering, say by subtracting the centroid from the TOAs of scatter broadened profiles will eliminate the GW signal as well. On the other hand, the recovered PBFs will be affected by scintillation and radiometer noise, so in order to extract parameters from the recovered PBFs, it is necessary to find the best fitting function, which in our case is a one-sided exponential. The disadvantage of this method, however, is that in realistic pulsar signals, the PBFs are not always one-sided exponentials with a sharp rising edge. Therefore, a different method needs to be developed in order to calculate scatter corrected TOAs that retain other delays. 


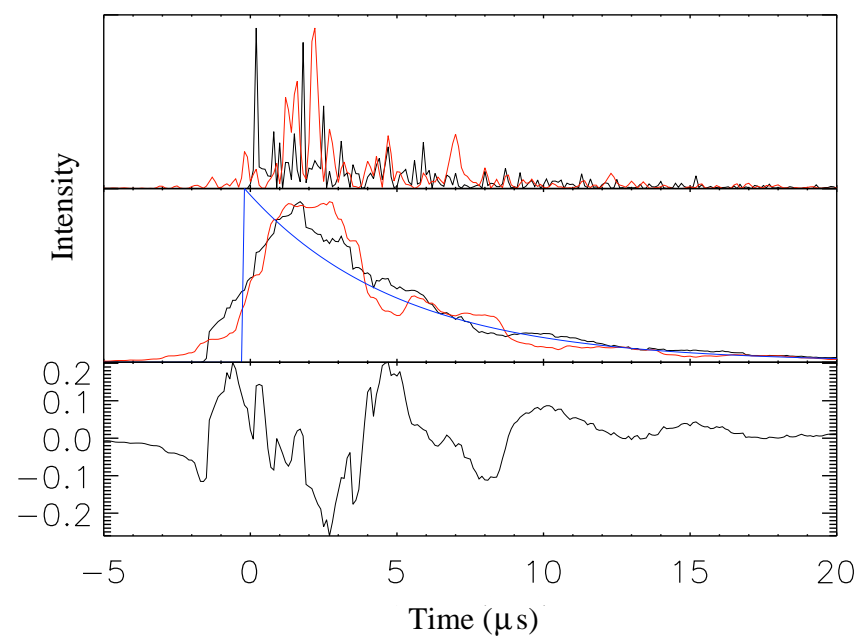

Figure 3.4: An example recovered intensity pulse broadening function $h_{I}(t)$ constructed from a cyclic spectrum (red curve) for an input scattering timescale of 5.2 $\mu \mathrm{s}$. For this realization, the scattering timescale estimated using the fitting technique is $5.3 \mu \mathrm{s}$. The input function is shown by the black curve and the best fitting one-sided exponential function is shown by the blue curve. The middle plots are smoothed versions of the top plots and the bottom plot shows the residual between the input and recovered PBFs.

We simulated average pulse profiles and cyclic spectra for 25 trials, with each trial representing a single epoch, with time-variable scattering, where the scattering timescale $\tau$ was drawn from a random distribution with a variance $1 \mu \mathrm{s}$. We have only considered cases in which $\tau<W$. The PBFs were recovered from cyclic spectra on each of these trials. These PBFs were smoothed by 10 samples, and a one-sided exponential function of the form $h_{I}(t)=\left|A U\left(t-t_{0}\right) \exp ^{-\left(t-t_{0}\right) / \tau^{\prime}}\right|^{2}$, where $A$ is the amplitude and other terms are as explained in Section 3.3, was fit to the smoothed PBFs. The best fitting parameters for amplitude $A$, time delay $t_{0}$ and scattering timescale $\tau$ of the recovered PBFs were determined though minimizing a $\chi^{2}$ grid search over the above three parameters. As shown in Figure 3.3, the intensity PBF with a scattering timescale of $5.3 \mu$ s input to the simulation is recovered with a 
scattering timescale of $5.2 \pm 0.4 \mu \mathrm{s}$.

In order to gauge the effect of the scattering correction on timing measurements, we first calculated the TOAs for the scatter-broadened pulse profiles on the 25 trial epochs using the Taylor algorithm (Taylor, 1992). This cross-correlation algorithm calculates the phase shift between the observed average profile and a high quality standard profile through an iterative, frequency-domain fitting algorithm to calculate a TOA. A scattered standard profile which has a scattering timescale equal to the mean of the input scattering times $(5 \mu \mathrm{s})$ was used for this purpose. The best fitting $t_{0}$ values were considered as the scatter-corrected TOAs. The residuals were calculated by subtracting the TOAs from those predicted by a simple timing model. The errors on the best fit parameters $t_{0}$ and $\tau$ were calculated from the $2 \times 2$ covariance matrix. The error on the residuals is the error of $t_{0}$.

Figure 3.5 shows the residuals before and after scatter correction for 25 trials. The scattering timescales of input one-sided exponential intensity PBFs ranged from $\sim 3.3 \mu$ s to $\sim 6.4 \mu \mathrm{s}$. A scattered standard profile which has a scattering timescale equal to the mean of the input scattering times $(5 \mu \mathrm{s})$ was used in the cross-correlation algorithm. This is a realistic assumption for actual PTA observations, as standard profiles are formed from averages of scattered observed profiles. The RMS of the residuals derived from the Taylor algorithm analysis for scatterbroadened average profiles have a standard deviation of $1 \mu$ s over the 25 trials of observation. When scatter corrected, the RMS of the residuals reduces to $359 \mathrm{~ns}$ from $1 \mu$ s. Profiles simulated with no scattering and scintillation give 'white-noise' RMS residuals of 23 ns. These results show that the RMS of the residuals calculated 


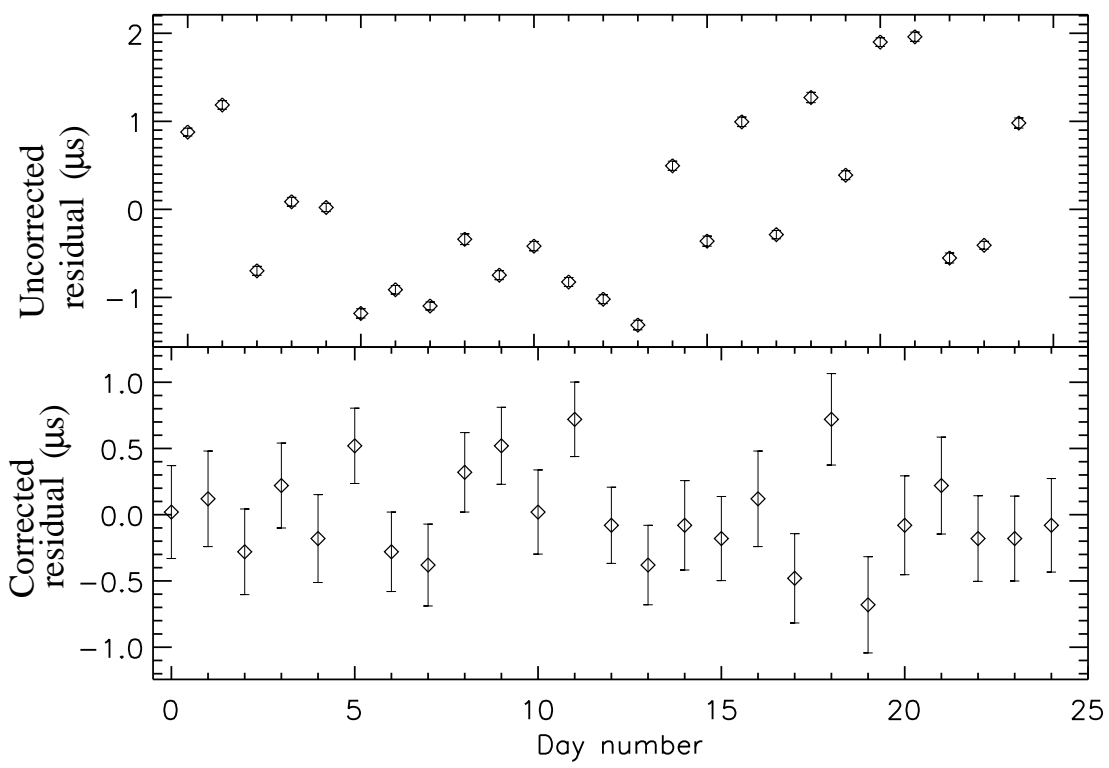

Figure 3.5: Top: Uncorrected residuals for 25 trials where the input scattering times were drawn from a random distribution with a mean of $5 \mu$ s and a standard deviation of $1 \mu \mathrm{s}$. The additive noise level was set to 0.5 times the average on-pulse power for a single pulse and $10^{4}$ pulses have been added to form the average profile of each trial. Bottom: Delays after applying the CS correction. The error bars of top and bottom panels are calculated from the $\chi^{2}$ minimization and the covariance matrix of the one-sided exponential fitting procedure respectively. The RMS of uncorrected and CS-corrected residuals are $970 \mathrm{~ns}$ and $359 \mathrm{~ns}$, respectively.

through the Taylor algorithm can be decreased significantly when the arrival times are scatter-corrected, approaching a factor of 2.7 improvement.

In the absence of other effects such as pulse-to-pulse jitter and red noise, TOA errors are dominated by radiometer noise that scales as $W /\left(S_{0} \sqrt{N}\right)$. Here $W$ is the width of the pulse profile, $S_{0}$ is the single pulse signal-to-noise-ratio, and $N$ is the number of pulses averaged. In order to test the correction scheme for various $\mathrm{S} / \mathrm{N}$ levels, defined as the ratio between the amplitude of the pulse peak and the RMS of the profile noise baseline, the number of pulses in the average profile was varied, averaging over $1 \times 10^{2}, 5 \times 10^{2}, 1 \times 10^{3}, 1.5 \times 10^{3}, 1 \times 10^{4}, 2 \times 10^{4}, 4 \times 10^{4}, 8 \times 10^{4}$, 
and $10^{5}$ pulses. We have used 16384 profile bins. The additive noise level, or the amount of instrumental noise, as a fraction of the peak of the pulse modulation function, was set to a constant value of 0.5 .

Figure 3.6 shows that the RMS of scatter-corrected residuals decreases with increasing $\mathrm{S} / \mathrm{N}$. We find that the shape of the recovered PBFs for low $\mathrm{S} / \mathrm{N}$ profiles whose $\mathrm{S} / \mathrm{N}$ is lower than $\sim 50$, or when the number of pulses averaged over is less than 100, starts to differ significantly from the input PBF. For typical NANOGrav observations, more than 500 pulses are averaged per TOA and resultant S/N values are typically greater than 100 (though not for all cases). Figure 3.7 shows how the $\chi^{2}$ of residuals varies as a function of profile S/N. In Figure 3.8, we show four example average profiles corresponding to different $\mathrm{S} / \mathrm{N}$, and in Figure 3.9 we show the input scattering timescales and the scattering timescales recovered from the best fitting one-sided exponential functions.

We have also assessed the effectiveness of the CS scatter correction for varying mean scattering times ranging from 3 to $17 \mu \mathrm{s}$. Figure 3.10 shows the RMS of residuals before scatter correction, RMS of residuals after scatter correction, and the ratio between the corrected and uncorrected RMS. For each value of the mean scattering time, the RMS of the input scattering times was set to be a factor of 0.2 of the mean scattering time, so that the RMS of scattering times increase with increasing mean scattering time, as typically seen in real pulsar signals (Hemberger \& Stinebring, 2008; Ramachandran et al., 2006). We find that the ratio between the CS corrected and uncorrected RMS residuals decreases with increasing mean scattering timescale due to the possibility of obtaining better fits to the recovered 


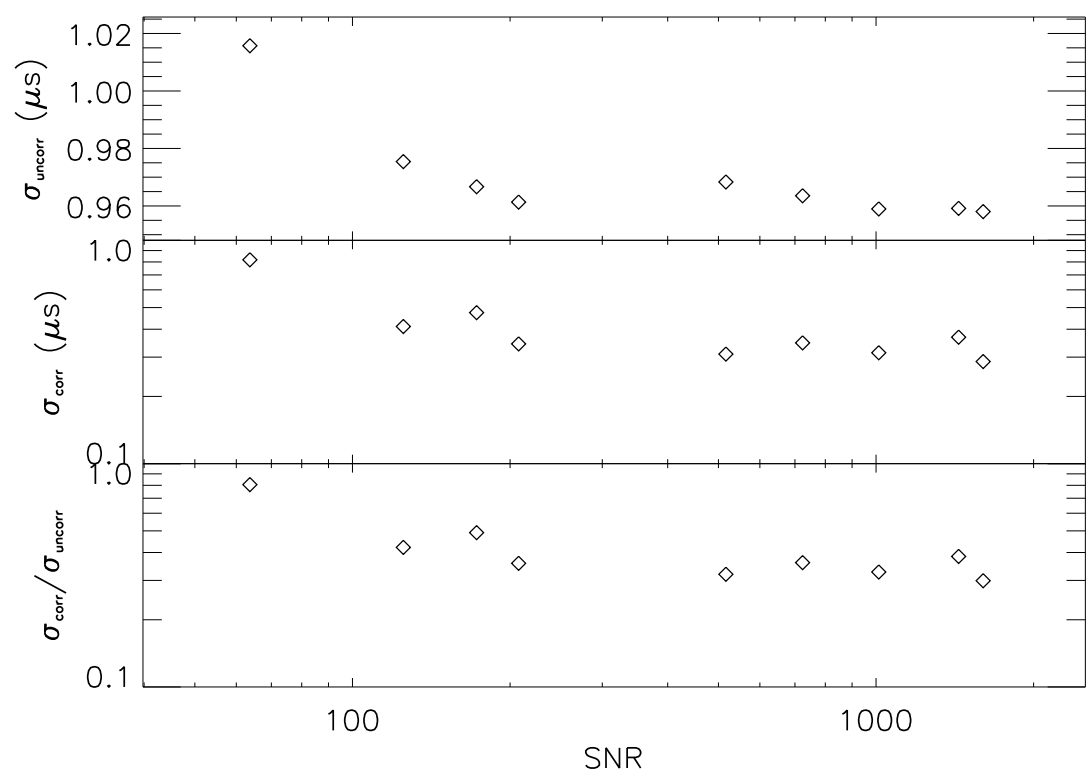

Figure 3.6: RMS of uncorrected residuals (top), RMS of CS-corrected residuals (middle), and the ratio between the RMS of CS-corrected residuals and the RMS of uncorrected residuals (bottom) as a function of average profile S/N. The RMS of uncorrected residuals ranges from 1.02 at $\mathrm{S} / \mathrm{N} 63.7$ to 0.95 at $\mathrm{S} / \mathrm{N}$ 1602.2. Each RMS value is calculated over 25 trials where the scattering timescale of each average profile was drawn from a uniform distribution with a mean of $5 \mu$ s and a standard deviation of $1 \mu \mathrm{s}$. The $\mathrm{S} / \mathrm{N}$ was varied by changing the number of pulses added. The number of pulses added is $1 \times 10^{2}, 5 \times 10^{2}, 1 \times 10^{3}, 1.5 \times 10^{3}, 1 \times 10^{4}, 2 \times 10^{4}$, $4 \times 10^{4}, 8 \times 10^{4}$, and $10^{5}$, respectively.

PBFs. Figure 3.11 shows how the $\chi^{2}$ of residuals varies as a function of mean scattering timescale.

These results are expected since even in an ideal situation the scatter correction scheme will be limited by the finite $\mathrm{S} / \mathrm{N}$ of the simulations. The width of a pulse $W_{a}$, composed of a noisy Gaussian with width $W_{i}$, convolved with a one-sidedexponential voltage $\mathrm{PBF}$ having a broadening timescale $\tau$, can be expressed as $W_{a} \approx \sqrt{W_{i}^{2}+\tau^{2}}$. As the scattering timescale $\tau$ increases and becomes comparable to the pulse width, shape changes come into play, in addition to time delays when cross-correlating the standard and observed profiles. 


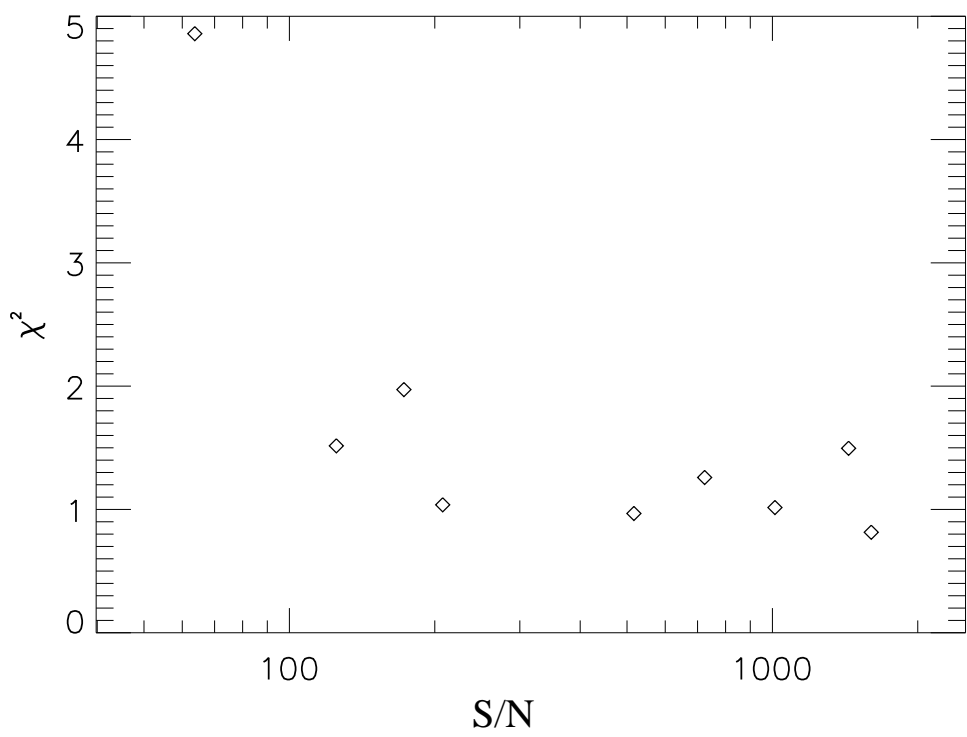

Figure 3.7: The $\chi^{2}$ of residuals as a function of average profile $\mathrm{S} / \mathrm{N}$, for the simulation in Figure 3.6.

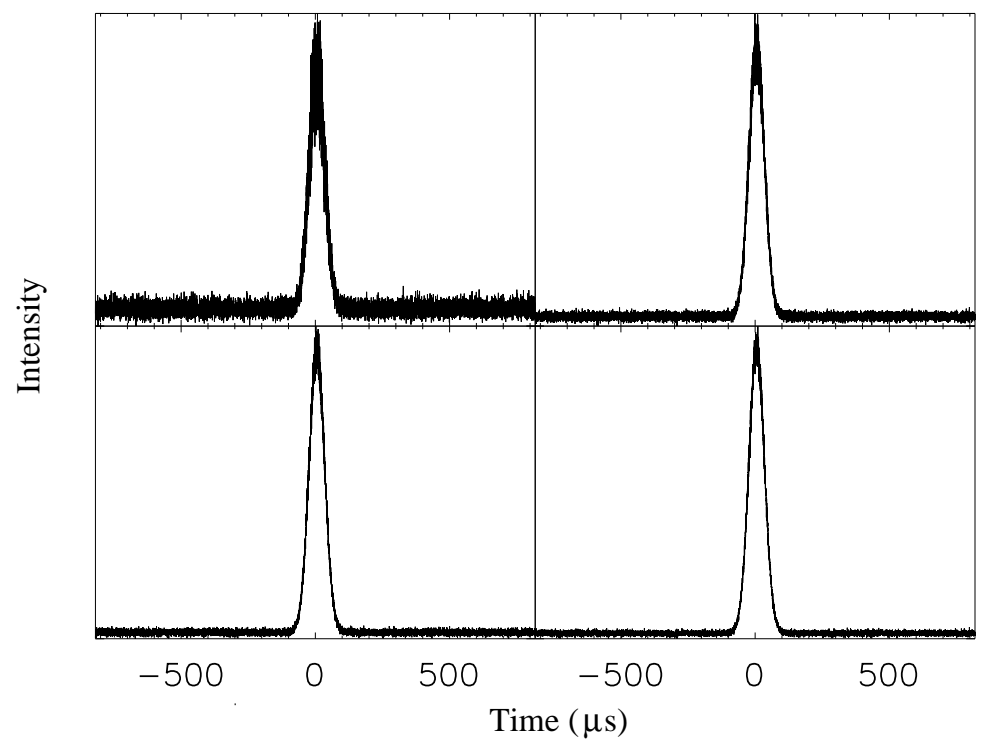

Figure 3.8: Average profiles with $\log (\mathrm{S} / \mathrm{N})$ values of, clockwise from top left, 1.8, 2.1, 2.2 and 2.3, corresponding to the lowest four $\mathrm{S} / \mathrm{N}$ data points in Figure 3.6. 

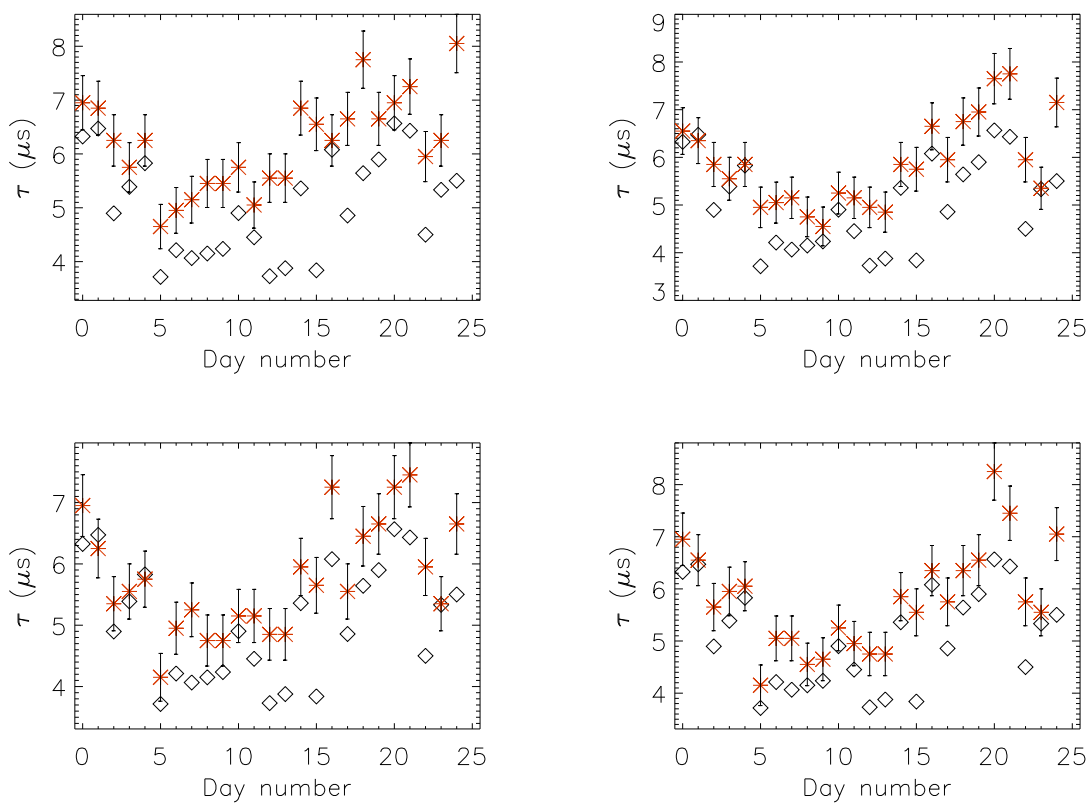

Figure 3.9: The input scattering timescales (diamonds) and the scattering timescales from the best fitting one-sided exponential functions (stars) for $\log (\mathrm{S} / \mathrm{N})$ values of, clockwise from top left, 1.8, 2.1, 2.2 and 2.3, corresponding to the lowest four $\mathrm{S} / \mathrm{N}$ data points in Figure 3.6. The error bars are calculated from the covariance matrix from the one-sided exponential fits. The fitting procedure tends to over-estimate the scattering timescale by roughly $20 \%$ in most cases. 


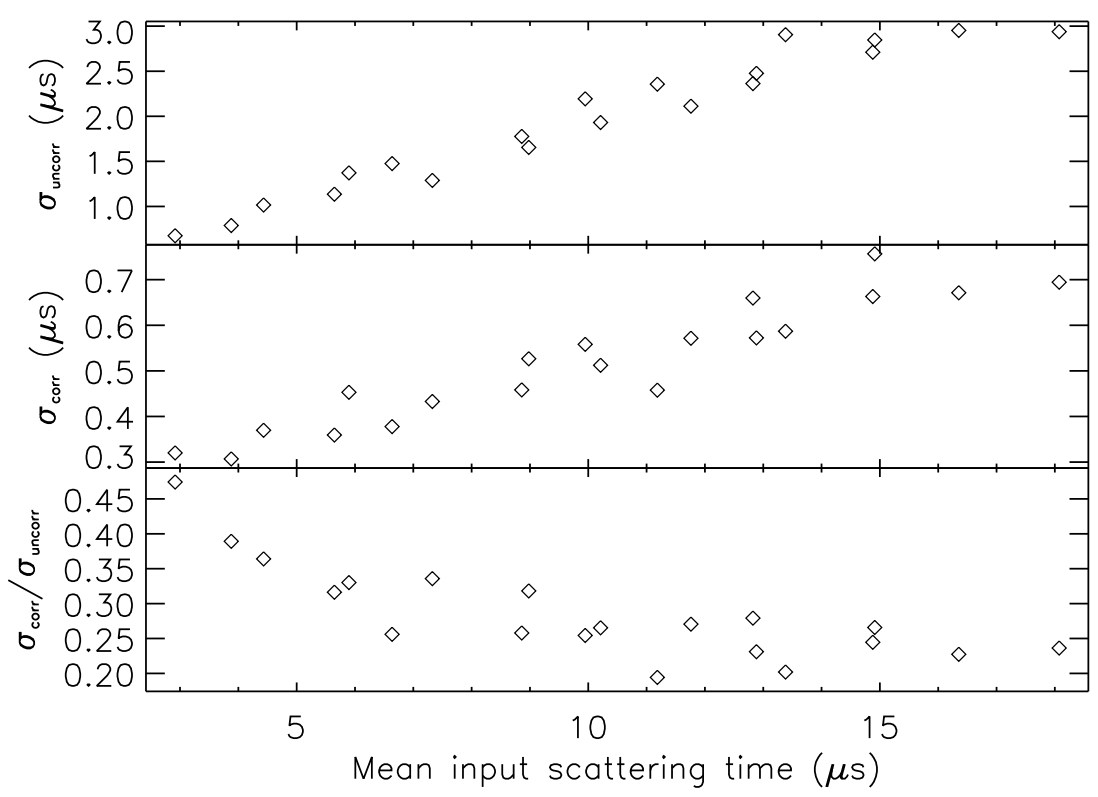

Figure 3.10: RMS of uncorrected residuals (top), RMS of CS-corrected residuals (middle), and the ratio between the RMS of CS-corrected residuals and the RMS of uncorrected residuals (bottom). The scattering times for each mean scattering time are drawn from a random distribution that has the given mean and a standard deviation equal to mean $/ 5$. Therefore, as the mean scattering time increases, the variation of the scattering time also increases as is the case in real pulsars. The additive noise level, or the amount of instrumental noise, as a fraction of the peak of the pulse modulation function, was set to 0.5 .

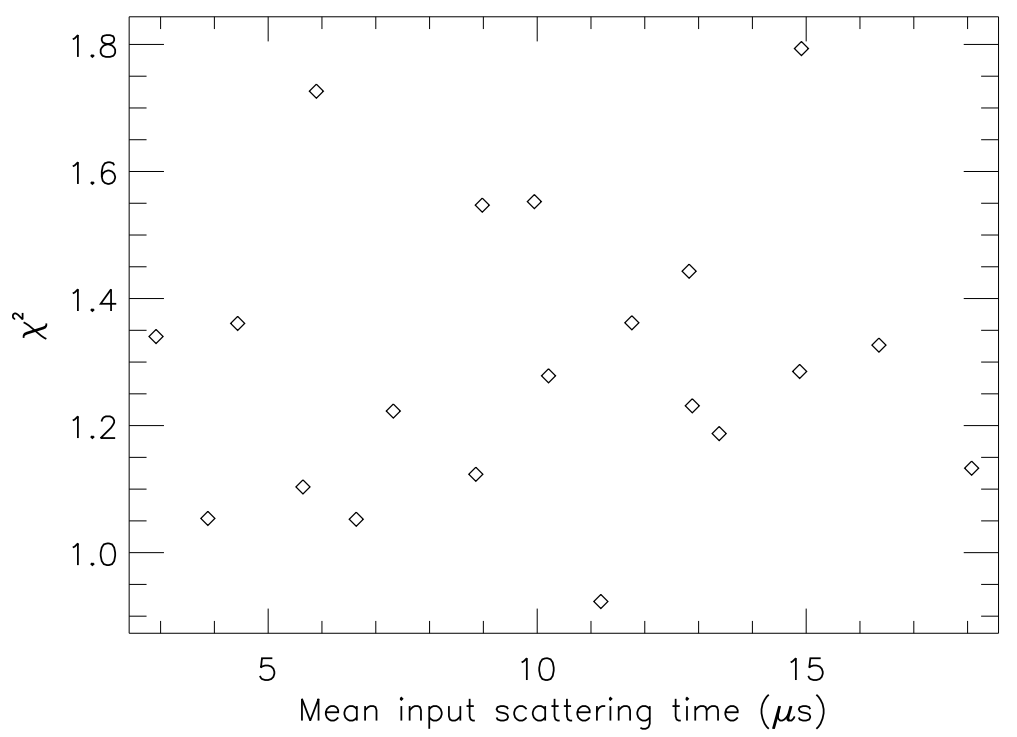

Figure 3.11: The $\chi^{2}$ of residuals as a function of the mean input scattering timescale, for the simulation in Figure 3.10. 


\subsubsection{Effects of pulse-to-pulse jitter}

Single pulses from some millisecond pulsars show random fluctuations in arrival phase which can be of the order of a pulse width. This is referred to as pulse phase jitter (Cordes \& Shannon, 2010). While pulses from PSR B1937+21 show jitter that contributes to a TOA uncertainty of 19 ns (Cordes \& Shannon, 2010), giant pulses from this object also exhibit jitter (Kinkhabwala \& Thorsett, 2000). PSRs J1713+0747 and J0437-4715, precisely timed PTA pulsars (epoch averaged RMS of 30 ns (Demorest et al., 2013) and 75 ns (Manchester et al., 2013)), are among the MSPs that show significant phase jitter that affect the TOA errors at levels of 65 ns and 105 ns (Cordes \& Shannon, 2010) respectively. In order to test the effect of phase jitter on the CS recovery of PBFs, we simulated jittered average profiles where the pulse phase fluctuations were drawn from a Gaussian random distribution, consistent with observations of jitter in normal pulsars.

Figure 3.12 shows the average profiles, single pulse profiles, and the recovered intensity PBFs for four values of the jitter parameter $F_{J}$, which is defined as $F_{J}^{2}=$ $1-\left(W_{1}^{2} / W_{a}^{2}\right)$ (Cordes \& Shannon, 2010), where $W_{1}$ and $W_{a}$ are the widths of single pulses and average profile respectively. Single pulses with widths 40, 30, 20, and 10 $\mu$ s and a scattering timescale of $5 \mu$ s were shifted in pulse phase from the center of the pulse to form a jittered average profile of width $\sim 40 \mu \mathrm{s}$. These correspond to jitter parameter values of $0.00,0.66,0.87$ and 0.97 . We have also computed the effect of scatter correction on jittered average profiles on 25 epochs for the above four jitter parameter values. We find that the RMS correction ratio $\left(\sigma_{\text {corr }} / \sigma_{\text {uncorr }}\right)$ 
increases from 0.3 to 0.4 as the jitter parameter increases from 0.0 to 0.97 . As seen in Figure 3.12, the PBFs are quite well recovered for all jitter parameter values. Given the fact that real MSP signals show small amounts of jitter (Cordes \& Shannon, 2010), the effect of phase jitter on impulse response recovery should be minimal.

\subsubsection{Presence of non-scattering delays}

As emphasized previously, $t_{0}$ as defined in Equation 3.10 may include other chromatic and achromatic delays. In order to verify that the scatter correction process does not remove other delays present, we added a GW signal to the simulated data and performed the scatter correction. The simulated GW signal has a period of 25 days and an amplitude of $10 \mu \mathrm{s}$. The GW signal was sampled at 25 trial epochs to obtain the delay values which were added as a time shift, to the scatter broadened simulated pulse profiles. The scattering timescales of these profiles were drawn from a random distribution with a variance of $1 \mu \mathrm{s}$, as outlined in Section 3.3.1. The corresponding PBFs were then recovered from the pulse profiles using the CS method. These profiles have been averaged over 10,000 single pulses and have a S/N of $\sim 500$. These PBFs were smoothed by 32 samples, and a one-sided exponential function was fit as explained in Section 3.3.1 to each recovered PBF.

In order to compare with the previous results of scatter-corrected residuals, we fit a sinusoid of the form $A \sin (2 \pi \mathrm{t} / \mathrm{T})$, where $\mathrm{t}, \mathrm{A}$ and $\mathrm{T}$ are time, amplitude and period respectively, to the best fit $t_{0}$ values. We then subtract the sinusoid from the residuals, in order to get the expected white residuals. We fit for the 

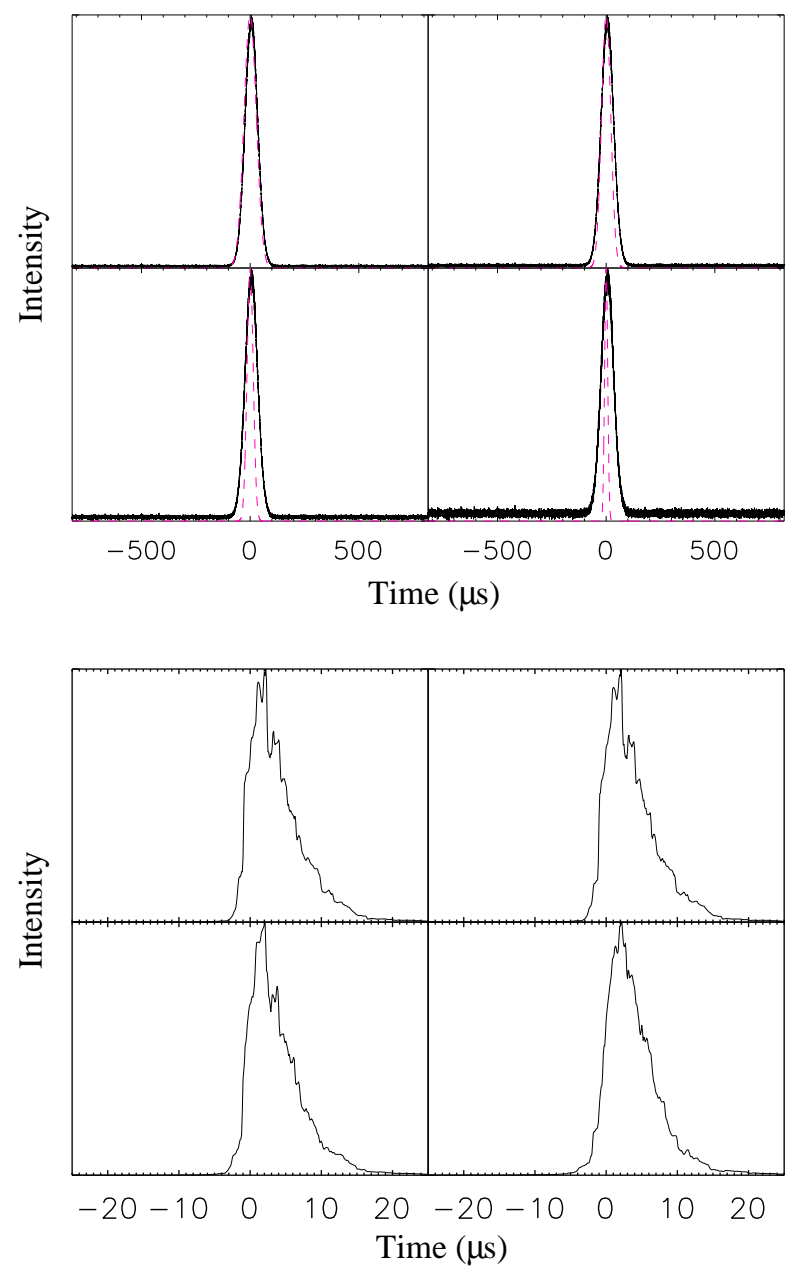

Figure 3.12: Top: Average profiles (black solid curve) from jittered single pulses (dashed curve) clockwise from top left, with widths of 40,30, 20, and $10 \mu \mathrm{s}$ and a scattering timescale of $5 \mu \mathrm{s}$. These correspond to jitter parameters of $0.00,0.66$, 0.87 and 0.97 . Bottom: Recovered intensity PBFs with scattering timescales of 5.1, $5.0,5.1$, and $4.6 \mu \mathrm{s}$. 


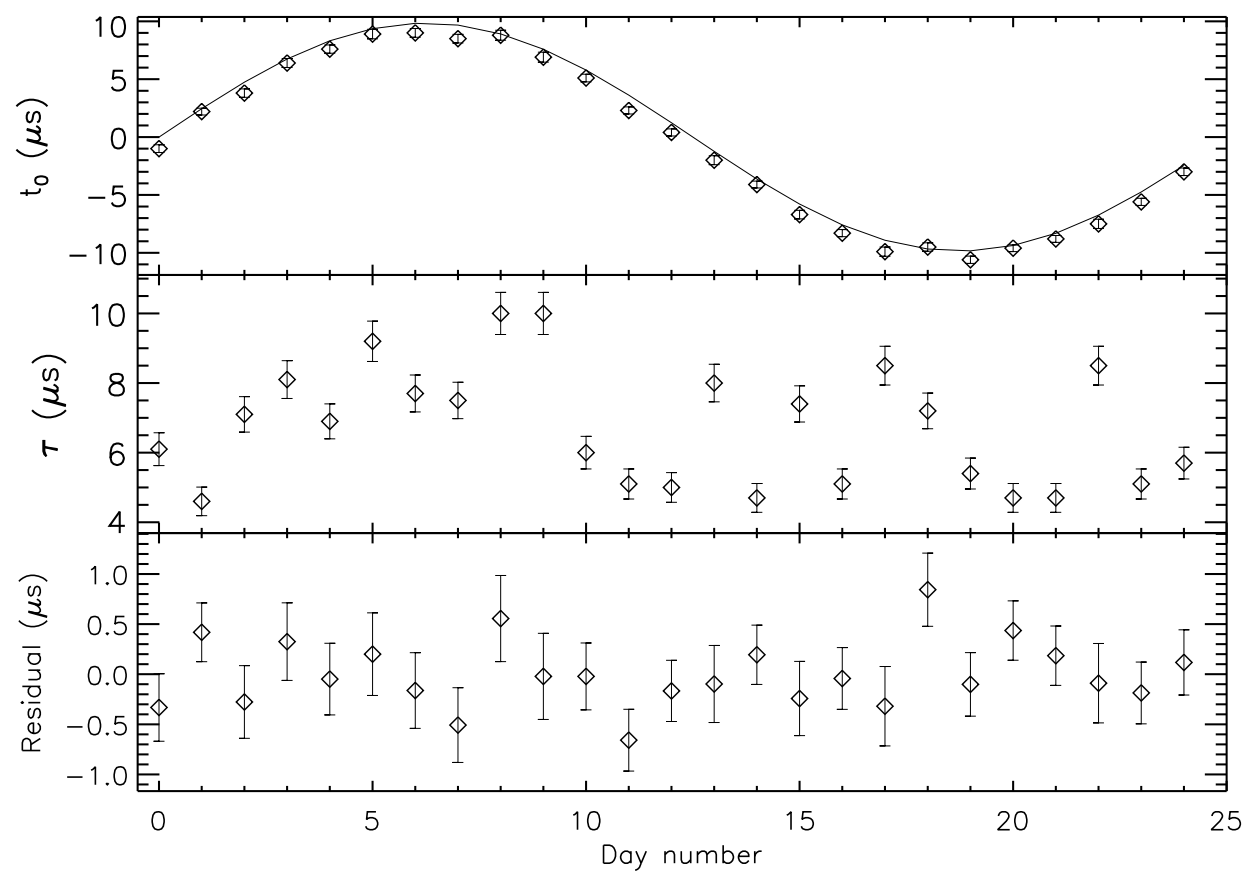

Figure 3.13: Best fitting $t_{0}$ (top), $\tau$ (middle) for a function of the form $h(t)=$ $A U\left(t-t_{0}\right) \exp ^{-\left(t-t_{0}\right) / \tau^{\prime}}$, where $\tau=\tau^{\prime} / 2$, and the white residuals which are calculated by removing the GW from the best fit $t_{0}$ values. The best fit sinusoid, which represents a gravitational wave signal, is overplotted in the top plot. The recovered GW signal has a period of $25.0065 \pm 0.0001$ days and an amplitude of $9.9552 \pm$ $0.0003 \mu \mathrm{s}$. The RMS of the white residuals is 340 ns. The RMS of the input scattering timescales for this case is $\sim 1 \mu \mathrm{s}$. The errors on $t_{0}$ and $\tau$ are calculated from the covariance matrix. The errors on the residuals are calculated from the errors on $t_{0}$ and the error of the best fitting sinusoid using error propagation.

period and amplitude of the sinusoid and find the best fitting values to be 25.0065 \pm 0.0001 days for period $\mathrm{T}$ and $9.9552 \pm 0.0003 \mu \mathrm{s}$ for amplitude A. The RMS of residuals after the GW signal was fit out is 340 ns. These results are illustrated in Figure 3.13, which shows the best fitting parameters $t_{0}$ and $\tau$, and the residuals when the GW signal is removed from the best fit $t_{0}$ values. The errors on the best fit parameters $t_{0}, \tau$ and the best fitting sinusoidal GW signal, were calculated from the $2 \times 2$ covariance matrix. The error on the residuals is the error of $t_{0}$.

Through an alternate method, frequency-dependent (chromatic) delays from 
achromatic delays such as those due to a gravitational wave can be separated though a carefully-designed multi-frequency timing analysis. What is gained with the CSapproach is that we retrieve a function for the PBF (as well as the TOA), whereas a traditional timing analysis would simply report a single number, the TOA. The shape and frequency-behavior of the PBF produced through the CS-analysis explored here can be used to separate chromatic and achromatic effects. But, a full implementation of that program requires a careful analysis of the form of the PBF due to various ISM propagation effects and is beyond the scope of this paper. Nevertheless, the additional information provided by the PBF function, which is itself frequency-dependent, should allow this separation to be made much more accurately than when only frequency-dependent TOAs (single numbers) are produced.

\subsection{Discussion}

We have used simulations to show that the CS method, based on electric field phase retrieval, can be used to accurately recover the complex voltage $\mathrm{PBF}, h(t)$, under realizable conditions for the brightest pulsars observed with 100-m class radio telescopes, for which the $\mathrm{S} / \mathrm{N}$ we have used here is applicable. For profiles containing a mean scattering delay of $5 \mu \mathrm{s}$ and an RMS variation of $\sim 1 \mu \mathrm{s}$, the CS-correction technique reduced the RMS variation to $\sim 359 \mathrm{~ns}$. This is a factor of $\sim 2.7$ decrease in the residual RMS. We also find that the ratio of pre to post scatter-correction RMS improves with increasing profile $\mathrm{S} / \mathrm{N}$. This implies that CS scatter correction is, not surprisingly, more effective on bright pulsars. The finite $\mathrm{S} / \mathrm{N}$ of the simulation sets 
a lower limit on the efficacy of the CS correction scheme. In the presence of a GW signal, in order to separate the scattering delays from the GW delays, it becomes necessary to fit a one-sided exponential function to the recovered PBF, in order to locate the rising edge of the function, which gives the scatter corrected TOA. The residuals obtained this way have an RMS of $340 \mathrm{~ns}$, corresponding to a factor of more than $\sim 2$ decrease in the residual RMS. Real pulsar signals will contain other delays, such as those due to refraction, that will cause $t_{0}$ of Equation 3.10 to be chromatic. In a production timing campaign, the PBFs reconstructed here would need to be analyzed further in a multi-frequency model from which terms with different frequency scalings - including the important frequency-independent term - would be extracted.

Out of the MSPs that exhibit jitter, jitter parameters (see Section 3.3.2 for definition) of $\sim 0.2-0.5$ for giant pulses of B1937+21 (Kinkhabwala \& Thorsett, 2000), 0.4 for pulses of J1713+0747 (Shannon \& Cordes, 2012), and 0.07 for pulses of J0437-4715 (Liu et al., 2012) have been observed. Our results show that onesided exponential PBFs with the expected timescales can be recovered from pulse profiles that show these amounts of jitter using this technique. Therefore the effect of pulse phase jitter on PBF recovery should be negligible.

It is important to note that the improvement in timing precision that we demonstrate is solely due to scattering time delay correction and not to pulse sharpening through the removal of scatter broadening. The advantage of this method is that it does not require prior assumptions of the shape of the PBF or the pulse shape, unlike the previously proposed PBF retrieval techniques that rely on prior 
assumptions of either one or both entities. The strong frequency dependence of scattering can help distinguish between ISM effects and other achromatic effects when multi-frequency observations are made. This is vital because one of the key applications for this technique is in the effort to detect GWs with pulsar timing. Since the GW signal is achromatic, we must be careful to prevent or minimize its inclusion in any correction of TOAs that is done in a dedicated PTA effort.

This problem is not limited to the PBF estimation technique presented here. It is inherent in separating the effects of ISM and other frequency-dependent delays from the achromatic signal of interest. Following the pioneering work of Demorest (2011) and Walker et al. (2013), our simulation takes a next step toward developing a production-quality chromatic correction technique. As we have emphasized, the CS-technique produces a TOA and a PBF function instead of a single number, the TOA. This provides the platform for a fuller multi-frequency analysis of the timing information embodied in $h(t ; \nu)$ which should certainly improve our ability to separate chromatic and achromatic influences on the pulse arrival time.

The simulated pulsar signal is scatter-broadened amplitude-modulated noise and includes scintillation effects. We have limited the analysis to this case in order to demonstrate the effect of scatter correction on improving timing residuals without the complications of other smaller effects. This technique can be applied to real pulsar signals whose phase information is preserved when recorded with a baseband setup. However, when applying this to real pulsar data we note that the scintle size, phase connection between scintles, and signal-to-noise ratio may need to be accounted for. Furthermore, when implementing on real pulsar data, the cyclic 
spectra calculated at radio frequencies $\nu$ within a bandwidth of $B$ will be valid within a region described by $|\alpha / 2|+|\nu|<B / 2$ (see Demorest, 2011, for details). Cyclic spectroscopy has so far been tested only on pulsar B1937+21 (Demorest, 2011), a bright pulsar which exhibits pronounced DM variations and pulse broadening variations (Ramachandran et al., 2006); the possibility of achieving a descattered pulse profile is shown in that paper.

We have taken a step forward to show the effect of scatter correction on pulsar timing. The application of CS to Arecibo baseband data of pulsar J1713+0747, which currently has the lowest RMS residuals for any NANOGrav pulsar (Demorest et al., 2013), will be presented in a future paper.

Scattering correction, when fully achieved, will allow higher precision pulsar timing, which will facilitate GW detection efforts using pulsars. It will also increase the number of MSPs able to be included in a pulsar timing array and will improve timing at lower frequencies. This should also improve timing of pulsars if found in the Galactic center region, currently limited due to scattering effects from turbulent plasma in these dense regions. 


\section{Chapter 4}

Single pulse properties of J1713+0747

\section{To be submitted to ApJ, with co-authors M. A. McLaughlin, S. Oslowski and others}

\subsection{Introduction}

Pulsar radiation comes from charged particles being accelerated along magnetic field lines and shows a wide variety of polarization properties. Linearly polarized light is generated as a result of the parallel (to the magnetic field line) and perpendicular components of the electric field $\left(\mathrm{E}_{\|}\right.$and $\mathrm{E}_{\perp}$ respectively) of the emitters $e^{+}-e^{-}$. Circular polarization is thought to be generated both by intrinsic mechanisms and/or by propagation effects (Melrose, 1994), where a time delay is introduced between $\mathrm{E}_{\|}$and $\mathrm{E}_{\perp}$ due to the refractive index (Greidanus \& Strom, 1990). The sense reversal often noted in the circular polarization is attributed to curvature radiation (Mitchel 1987) and therefore considered a signature of intrinsic emission. A smooth S-shaped variation is expected in the position angle (PA), which is the angle between the rotation axis and the magnetic field line, as the pulsar beam crosses our line of sight, sweeping through different magnetic field lines. However, many pulsars show discontinuities and rapid jumps in the PA which can be both orthogonal and non-orthogonal. Backer et al. (1976) account for these by invok- 
ing two orthogonal modes of emission. Both these modes can occur simultaneously (superposed) or a single mode can be emitted at a given instant (disjoint).

MSPs have small light cylinders which imply large polar cap sizes and larger than usual half opening beam angles $\rho$ that do not follow the general $\rho \propto P^{-0.5}$ relation, where $\mathrm{P}$ is the pulsar period, and a frequency evolution that is different from normal pulsars (Kramer et al., 1998). MSPs in general show a higher degree of polarization and more complex PA variations than those seen in canonical pulsars (Xilouris et al., 1998). The weaker magnetic fields of MSPs could imply particle gyration leading to more circular polarization, pointing towards possible deviations from a dipolar field structure, even though J0437-4715 studies are suggestive of similar emission mechanisms for normal pulsars and MSPs. (Jenet et al., 1998). Regardless of these differences, both normal pulsars and MSPs show similar complexity in profiles and similar spectral indices, implying similar magnetic field structure and emission conditions that could include outer gaps (Kramer et al., 1998). Therefore the study of polarization properties, more specifically of single pulses, is important from an emission mechanism standpoint as it will help verify these ideas.

PSR J1713+0747 is the brightest and one of the best timed NANOGrav pulsars, with a flux density of $6.3 \mathrm{mJy}$ at $1.4 \mathrm{GHz}$ and epoch averaged residuals with an rms of 30 ns (Demorest et al., 2013) from predominantly white processes (Verbiest et al., 2009). This remarkable timing precision makes this a key object in understanding the noise floor or the ultimate level of precision achievable and sources of TOA error. Among possible factors affecting timing of pulsars at these sub- 
nanosecond levels in addition to radiometer noise could be pulse-to-pulse variability (phase jitter) and ISM effects such as diffractive scintillation. There is evidence for pulse phase jitter in this pulsar (Shannon \& Cordes, 2012) that accounts for at least $20 \mathrm{~ns}$ in the rms timing residuals. Pulse broadening due to ISM effects could be of the order of a few nanoseconds, considering typical scintillation bandwidths of 2 $\mathrm{MHz}$.

Single-pulse studies have, in the past, made it possible to identify phenomena such as moding, drifting, and nulling in canonical pulsars, in addition to being crucial in understanding emission physics (Rankin, 1986). There is evidence for single-pulse polarization varying dramatically from the average profile (Cordes \& Hankins 1977). Single pulses from some pulsars show more circular polarization than linear polarization in their subpulses (Stinebring et al., 1984). Non-geometrical swings in the PA can be expected in single pulses (Greidanus \& Strom, 1990), which get lost in the averaging process. High fractional linear polarization is attributed to non-overlapping dipole field lines which implies less depolarization, and therefore, single-pulse studies could provide interesting information in this regard. Singlepulse studies of MSPs have been sparse due to signal-to-noise ratio limitations due to their low flux (Kramer et al., 1998) and data acquisition requirements such as the need for high time resolution sampling.

The few existing studies conducted in the past have revealed highly linearly polarized single pulses and sub-pulse microstructure in J0437-4715 (Jenet et al., 1998), giant pulses from B1937+21 (Jenet et al., 2001), and jittering pulses from J1713+0747 (Shannon \& Cordes, 2012) and J0437-4715 (Jenet et al., 1998). Polar- 
ization properties of single pulses have only been conducted on J0437-4715 (Jenet et al., 1998) and on giant pulses of B1937+21 (Zhuravlev et al., 2013).

\subsection{Observations and data processing}

The data used in this analysis are the Arecibo observations of J1713+0747 performed on the $22^{\text {nd }}$ June 2013 as part of the 24-hour Global campaign (Dolch et al., 2014). The pulsar was observed at $1.4 \mathrm{GHz}$ for $\sim 1.6$ hours with the PUPPI backend and a bandwidth of $800 \mathrm{MHz}$. In order to obtain calibration data, prior to the pulsar observation, a $25 \mathrm{~Hz}$ winking cal signal was turned on for 2 minutes while pointing at the pulsar. The pulsar was then observed, retaining the same receiver gains. The data used here were obtained in the coherent search mode as non-folded intensity data.

The data were coherently dedispersed using dspsr. Single pulses were obtained using PSRCHIVE (Hotan et al., 2004) psrspa and were visually inspected for phase and shape consistency. RFI removal was done using pazi, by visually inspecting chunks of data that showed off-pulse flux that was greater than $4 \sigma$, where $\sigma$ is the off-pulse RMS. Polarization calibration modeling (pcm) as outlined in van Straten (2004) was used to calibrate the data. This uses the pulsar polarization at multiple parallactic angles to constrain the instrumental responses such as the absolute gain, the differential gain and phase and ellipticities of receptors. The model then uses the noise diode signal to constrain rotation about the line of sight. See Section 1.5.1 for more information about polarization calibration. 


\subsection{Data analysis and results}

Stokes parameters I, Q, U, V are calculated from the data products and the degree of linear polarization is calculated as $L=\sqrt{Q^{2}+U^{2}}$. The polarization position angle is defined as P.A. $=0.5 \tan ^{-1}(U / Q)$. Fractional polarizations $(L / I$ and $V / I)$ were calculated at every pulse longitude. The PA is calculated when the linear polarization is greater than three times the off-pulse rms $\left(L>3 \sigma_{L}\right)$.

Figure 4.1 shows the histograms of fractional linear and circular polarizations at four pulse phases near the on-pulse region and the distribution of the position angle as a function of pulse phase. We find that the single pulses of J1713+0747 are highly linearly polarized, with the peak fractional linear polarization histogram maximizing at 0.66 at phase 0.76 , located within the central region of the pulse profile. PA variations in single pulses are complex, sometimes showing orthogonal/nonorthogonal jumps. There is no evidence for orthogonal polarization modes in the PA histogram of this pulsar.

\subsubsection{Timing bright pulses}

The TOA error due to radiometer noise $\Delta t_{R N}=\mathrm{W} /(\mathrm{S} / \mathrm{N} \sqrt{\mathrm{N}})$, where $\mathrm{W}, \mathrm{S} / \mathrm{N}$ and $N$ are pulse width, the average signal to noise ratio of a single pulse and the number of pulses respectively. As Jenet et al. (1998) demonstrated for J0437-4715, as the pulse amplitude increases with decreasing width, if there are no jitter effects, considering the brightest pulses should result in a narrower profile. Figure 4.2 shows a bright single pulse and the average profile where the smaller width of the single 


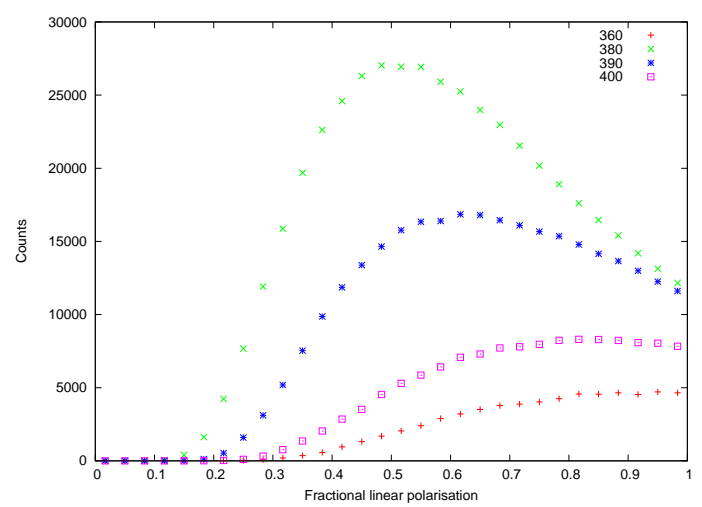

(a)

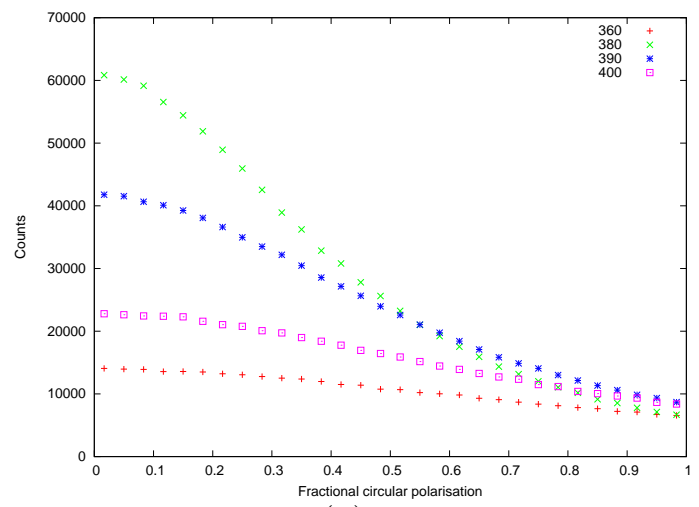

(b)

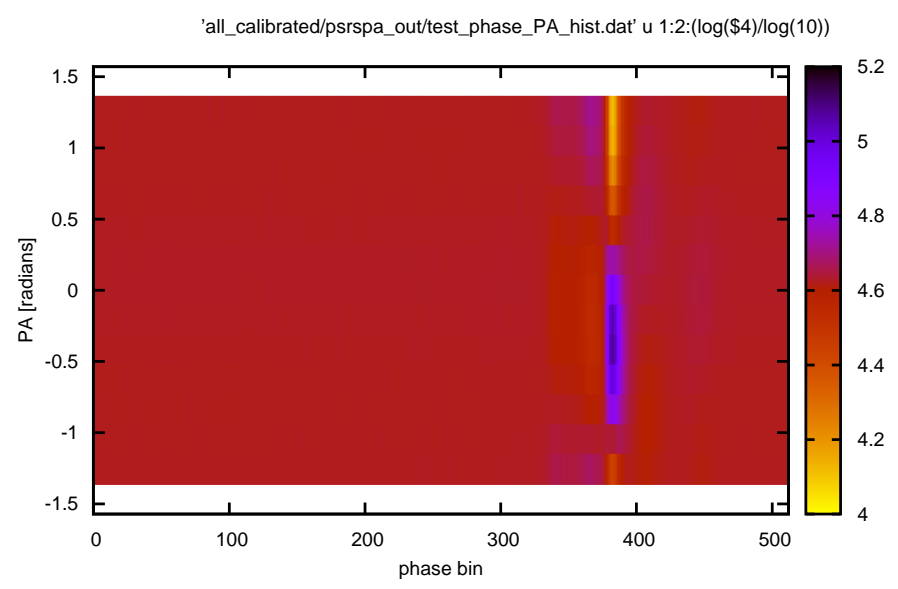

(c)

Figure 4.1: The histograms of the degree of linear polarization (a), degree of circular polarization (b), and the distribution of the position angle as a function of pulse phase for all single pulses (c). 


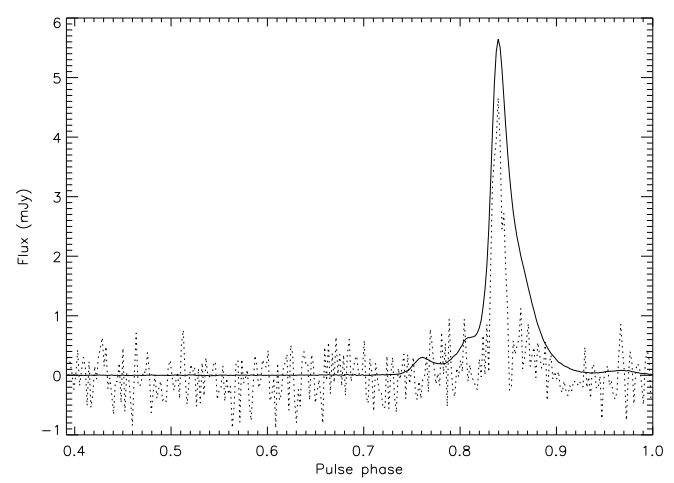

Figure 4.2: A bright single pulse (dotted) of J1713+0747 plotted together with average profile (solid). The average profile has been multiplied by six for comparison.

pulse is apparent. Composite profiles constructed by adding a few bright pulses for this purpose and were used to calculate TOAs and the residuals were compared with the TOA residuals of profiles averaged over a few minutes. Table 4.1 lists the half widths, $\mathrm{S} / \mathrm{N}$ and the RMS residuals obtained with these composite profiles. Comparing the widths and $\mathrm{S} / \mathrm{N}$ of 120 second integration profiles and 500 bright pulse composite profiles, for example, it can be seen that the width reduces by a factor of $\sim 4$. However the $\mathrm{S} / \mathrm{N}$ also reduces by $\sim 6$ so the expected RMS residuals should go up by a factor of $\sim 1.5$. However the measured RMS increases by a factor of $\sim 2.6$. This could be due to the role of jitter as the bright pulses are more jittered (Shannon \& Cordes, 2012).

Figure 4.3 shows a two dimensional image of the RMS residuals as a function of pulse $\mathrm{S} / \mathrm{N}$ and the number of pulses combined to form the profile. All single pulses between the given $\mathrm{S} / \mathrm{N}$ limits are selected and combined to form composite profiles. The TOAs for these composite profiles are then used to calculate RMS residuals. The lowest RMS of 301 ns occurs when pulses with S/N between 10 and 


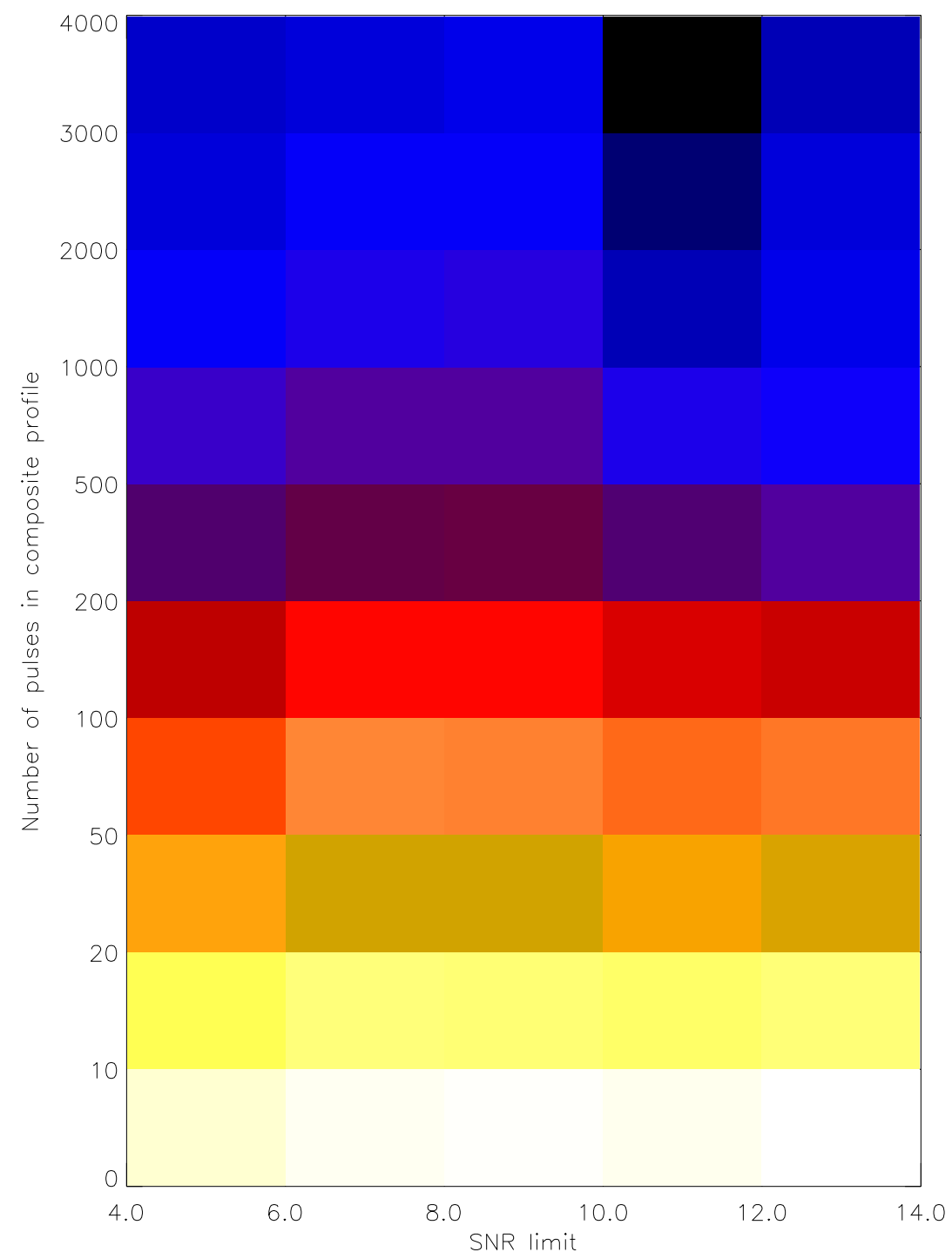

(a)

Figure 4.3: The RMS residuals as a function of $\mathrm{S} / \mathrm{N}$ limit and the number of bright pulses in the composite profile. The darker regions correspond to lower RMS values. The lowest RMS of $301 \mathrm{~ns}$ occurs when pulses with $\mathrm{S} / \mathrm{N}$ between 10 and 12 are picked and 4000 pulses are added together. 
Table 4.1: The length of time or the number of bright single pulses that went into the composite profile, the width, $\mathrm{S} / \mathrm{N}$ and the RMS timing residuals from the composite profiles. The half width of a typical single pulse is $0.035 \mathrm{~ms}$ with a $\mathrm{S} / \mathrm{N}$ of $\sim 12$.

\begin{tabular}{llll}
\hline Integration & $\mathrm{W}(\mathrm{ms})$ & $\mathrm{S} / \mathrm{N}$ & $\mathrm{RMS}(\mu \mathrm{s})$ \\
\hline 120 s integration & 0.084 & 1037.84 & 0.248 \\
200 single pulses & 0.022 & 96.5 & 0.748 \\
500 single pulses & 0.022 & 154.8 & 0.664 \\
\hline \hline
\end{tabular}

12 are picked and 4000 pulses are added together.

\subsection{Discussion}

We have presented the polarization properties of single pulses from J1713+0747. The analysis shows that the pulsar's emission is highly linearly polarized. The absence of orthogonal jumps in the PA and the fact that the emission is highly linearly polarized suggests that a single mode of emission dominates in this pulsar. Furthermore, it is seen that the single pulses from the outskirts of the profile have higher linear polarization. This can be interpreted based on the fact that the conal emission in this pulsar is almost $100 \%$ linarly polarized, so the linear polarization in the outer phase bins, away from the phase bin corresponding to the fiducial point, will be more linearly polarized.

Pulsar emission is attributed to charged particle acceleration along curved field lines, which is known as curvature radiation. Mitra et al. (2009) suggests that curvature radiation generated by bunches of solitons, which are pulse waves in plasma that exhibit characteristics of particles, can be responsible for the emission of pulsars. This is because only curvature radiation, as apposed to maser emission 
will produce PA variations in single pulses, that will follow the mean PA variation over the pulse longitude. Orthogonal modes of polarization have been invoked to explain the behaviour of the PA sweep that differs from the RVM s-shape. Also, the observation of a single mode of emission can be attributed to the extraordinary wave polarized perpendicular to the plane defined by the magnetic field line and the wave vector. The ordinary wave polarized in the plane of the field lines gets damped as it interacts with the magnetosphere, thereby emitting in a single mode.

We have also tested the possibility of timing the pulsar using composite profiles made up of bright pulses. However, our results show that this does not improve the RMS down to desired levels. Shannon \& Cordes (2012) showed that J1713+0747 shows pulse-phase-jitter that corresponds to 20 ns RMS in the timing residuals and that brighter pulses are more jittered. Therefore, even though bright pulse timing does not work for this pulsar, there may be other pulsars to which this technique might be applicable. 


\section{Chapter 5}

\section{Conclusions and future work}

This work was based on two separate topics on two pulsar classes, namely RRATs and MSPs. In Chapter 1 we introduced pulsars, described the different pulsar categories, discussed emission mechanisms and the basic characteristics. We also laid the foundation for pulsar timing, gravitational wave detection with MSPs and the effect of the ISM in this chapter. Chapters 2, 3 and 4 were dedicated to RRATs, ISM scatter-correction, and single-pulse studies of MSP J1713+0747 as applicable for PTAs respectively.

In Chapter 2 we searched for periodicities in RRATs' pulse arrival times and detection rates and compared the pulse sequences with random and uniform distributions. Several ideas have been presented about the nature of emission from RRATs such as the presence of a circumstellar asteroid belt or that they may be extreme nulling pulsars. Determining the time variability and/or periodicity of the RRAT pulses is therefore an important diagnostic of the RRAT emission mechanism. We found six of RRATs to show significant periodicities in their arrival times, ranging from hours to days. Based on the periodicity in pulse detection rate found in RRAT J1913+1330 and its timing residuals, we were able to constrain the mass of a possible planet to be of the size of the earth. In order to gauge the reality of the periodicities, the time series of detections and non detections were randomized 
by placing the pulses randomly within the observation windows and repeating the analysis. No periodicities were found with significance greater than $30 \%$ in any of these randomised time series. It was concluded that they are real, but further testing is needed to determine their origin. Furthermore we searched for any clustering present in the pulse sequences of these RRATs and found the same six RRATs to show non-random behavior. Exploration of the transient sky is fast-emerging area in radio astronomy. With major telescopes such as LOFAR, FAST, ASKAP, MeerKAT, MWA and SKA being dedicated for these searches, hundreds of RRAT sources will be discovered in the next few years. Applying these techniques to a larger sample will allow us to develop accurate models for emission mechanism and perhaps external environments of these sources.

In Chapter 3 we investigated the possibility of using cyclic spectroscopy (CS) to identify the ISM scattering delays pulsar data. We simulated the pulse profile as an amplitude modulated noise signal convolved with an ISM pulse broadening function $(\mathrm{PBF})$ and showed that $\mathrm{CS}$ can be used to recover the PBFs. In order for this technique to be useful for PTAs, we investigated the possibility of correcting the effect of scatter-broadening in pulse profiles using the recovered PBFs. For the simple thin screen model that we used in our simulations, scatter-corrected TOAs were obtained by considering the location of the rising edge of the recovered PBF. Furthermore, we found that the PBF recovery and scatter-correction depend on the profile S/N, suggesting that CS will be applicable only for bright pulsars.

Some NANOGrav pulsars show large amounts of scattering in their profiles and large RMS residuals unexplainable through radiometer noise. Some have steep 
spectra, which means that they could be good candidates to test timing at low frequencies. If sufficiently bright MSPs are found, this technique can be used to reliably deconvolve the intrinsic pulsar signal from the PBF. As mentioned in Chapter 3, for realistic pulsar signals, the recovered PBFs will have to be analyzed at multiple frequencies in order to determine the scattering timescales because they may not be simple one-sided exponential functions and may include other chromatic delays. Observing with large telescopes will be one way obtaining increased $\mathrm{S} / \mathrm{N}$ in pulsar signals. Therefore, MSPs observed with the next generation telescopes and future PTAs may greatly benefit from these techniques. Given the searching and timing capabilities achievable by the SKA, with thousands of potential MSP in the southern hemisphere and very low nanosecond level RMS residuals, PTA science with the SKA will be revolutionary. As more distant pulsars are found with the SKA, correcting for ISM effects will become important. The $\mathrm{S} / \mathrm{N}$ of these distant pulsars will be higher with the SKA and furthermore, PTAs will be able to select the brightest pulsars out of the thousands of MSPs that will be discovered. In addition, there are ideas on using interstellar scattering to determine pulsar distances with VLBI techniques (Pen et al., 2014). These efforts could also benefit from CS. As secondary science, since the effects discussed here arise from small scale structures in the size range $10^{6}-10^{12} \mathrm{~m}$, they could be applicable for studies probing the structure of the ionized ISM.

In Chapter 4 we investigated polarization properties of single pulses in order to understand the emission mechanism. We searched for orthogonal modes of emission using single pulses of J1713+0747 and also test the possibility of using bright pulses 
to time the pulsar. We found that the emission is highly linearly polarized and that a single mode of emission dominates in this pulsar. Single-pulse polarimetry of MSPs could be useful in ruling out different emission models and may offer new insights to physical phenomena occurring in dense and high gravity environments. Since the only study that exists on MSP single-pulse polarimetry of J0437-4715 points towards a single mode of emission, similar studies of many more MSPs will help us understand the peculiar shapes and jumps seen in the PA. On a PTA point-of-view, single-pulse studies of MSPs are also important to reveal information regarding the noise floor of the pulsar.

The Global Campaign was performed for this goal of identifying the processes that limit the timing precision of J1713+0747. Given that pulse-to-pulse jitter contributes to the noise budget by large amounts, it is necessary to identify and characterize the various noise processes. Until reliable methods of accounting for jitter are developed, techniques like timing pulsars with selective pulses could be potentially useful for mitigating this effect. Even though single pulse detection from MSPs is currently limited to a few objects, the SKA will permit this for many more objects, where these techniques will be applicable. 


\section{Appendix A}

\section{Signal to Noise Ratio for CS Reconstruction}

With the ISM acting as a linear filter, consider a frequency dependent time delay $\tau$ that produces a phase slope across the band. The signal we are trying to detect is the change in phase for a frequency offset of $\alpha_{n}$. This is

$$
\Delta \hat{\Phi}_{S_{y}}=2 \pi \tau \alpha_{n} .
$$

The estimator of this signal can be expressed in terms of the real and imaginary parts of a particular harmonic of the cyclic spectrum $S_{y}(\nu)$ as,

$$
\hat{\Phi}_{S_{y}}(\nu)=\arctan \left\{\frac{\operatorname{Im}\left\langle S_{y}(\nu)\right\rangle_{T}}{\operatorname{Re}\left\langle S_{y}(\nu)\right\rangle_{T}}\right\},
$$

where the angle brackets indicate averaging over $T$, the total integration time. Since the phase advance $\Delta \hat{\Phi}_{S_{y}} \ll 1$, the uncertainty in $\hat{\Phi}_{S_{y}}$ can be approximated as

$$
\delta \hat{\Phi}_{S_{y}} \approx \frac{\delta\left(\operatorname{Im}\left\langle S_{y}(\nu)\right\rangle_{T}\right)}{\left\langle\left|S_{y}(\nu)\right|\right\rangle_{T}}
$$


The uncertainty in the imaginary part of the cyclic spectrum is dominated by additive noise because $\sigma_{n}^{2} / \sigma_{p}^{2} \gg 1$ and can be defined as $\delta\left(\operatorname{Im}\left\langle S_{y}(\nu)\right\rangle_{T}\right)=\sigma_{I}$ with

$$
\sigma_{I}=\frac{\sigma_{n}^{2}}{\sqrt{N_{p} N / 2}}
$$

where $N$ and $N_{p}$ are the number of samples in the pulse period and the number of pulses, respectively. From Equation 3.8 the amplitude of the cyclic spectrum $\left|S_{y}\left(\nu ; \alpha_{n}\right)\right|=\left|c_{n}\right| \sigma_{p}^{2}$, so

$$
\delta \hat{\Phi}_{S_{y}} \approx \frac{\sigma_{n}^{2}}{\left|c_{n}\right| \sigma_{p}^{2} \sqrt{N_{p} N / 2}}
$$

Using Equations $\mathrm{A} 1$ and $\mathrm{A} 5$, the signal-to-noise ratio $\left(\mathrm{S} / \mathrm{N}_{\tau}\right)$ can now be expressed as,

$$
\mathrm{S} / \mathrm{N}_{\tau}=\frac{\tau}{\delta \tau}=\frac{\Delta \hat{\Phi}_{S_{y}}}{\delta \hat{\Phi}_{S_{y}}}=\frac{2 \pi \tau \alpha_{n}\left|c_{n}\right| \sigma_{p}^{2} \sqrt{N_{p} N / 2}}{\sigma_{n}^{2}}
$$

which because $\alpha_{n}=n / P$ yields the result $\mathrm{S} / \mathrm{N} \propto \mathrm{n}\left|\mathrm{c}_{\mathrm{n}}\right|$. 


\section{Bibliography}

Antoni J., 2007, Mechanical Systems and Signal Processing, 21, 597

Backer D. C., Kulkarni S. R., Heiles C., Davis M. M., Goss W. M., 1982, Nature, 300,615

Backer D. C., Rankin J. M., Campbell D. B., 1976, Nature, 263, 202

Bailes M., Johnston S., Bell J. F., Lorimer D. R., Stappers B. W., Manchester R. N., Lyne A. G., D’Amico N., Gaensler B. M., 1997, ApJ, 481, 386

Bates S. D., Lorimer D. R., Verbiest J. P. W., 2013, MNRAS, 431, 1352

Bhat N. D. R., Cordes J. M., Camilo F., Nice D. J., Lorimer D. R., 2004, ApJ, 605, 759

Bhat N. D. R., Cordes J. M., Chatterjee S., 2003, ApJ, 584, 782

Bhattacharya D., van den Heuvel E. P. J., 1991, Phys. Rep, 203, 1

Burke-Spolaor S., Bailes M., 2010a, MNRAS, 402, 855

Burke-Spolaor S., Bailes M., 2010b, MNRAS, 402, 855

Burke-Spolaor S., Bailes M., Johnston S., Bates S. D., Bhat N. D. R., Burgay M., D’Amico N., Jameson A., Keith M. J., Kramer M., Levin L., Milia S., Possenti A., Stappers B., van Straten W., 2011, MNRAS, 416, 2465

Camilo F., Ransom S. M., Halpern J. P., Reynolds J., Helfand D. J., Zimmerman N., Sarkissian J., 2006, Nature, 442, 892 
Chen K., Ruderman M., 1993, ApJ, 408, 179

Coles W. A., Rickett B. J., Gao J. J., Hobbs G., Verbiest J. P. W., 2010, ApJ, 717, 1206

Cordes J. M., 1976, ApJ, 210, 780

Cordes J. M., Lazio T. J. W., 2002, arXiv:astro-ph/0207156

Cordes J. M., Rickett B. J., 1998, ApJ, 507, 846

Cordes J. M., Shannon R. M., 2008, ApJ, 682, 1152

Cordes J. M., Shannon R. M., 2010, arXiv:1010.3785

Cordes J. M., Shannon R. M., 2012, ApJ, 750, 89

Damour T., Vilenkin A., 2005, Phys. Rev. D, 71, 063510

Demorest P. B., 2011, MNRAS, 416, 2821

Demorest P. B., Ferdman R. D., Gonzalez M. E., Nice D., Ransom S., Stairs I. H., Arzoumanian Z., Brazier A., Burke-Spolaor S., Chamberlin S. J., Cordes J. M., Ellis J., Finn L. S., Freire P., Lommen A. N., McLaughlin M., Palliyaguru N., 2013, ApJ, 762, 94

Deneva J. S., Cordes J. M., McLaughlin M. A., Nice D. J., Lorimer D. R., et al., 2009, ApJ, 703, 2259

Deshpande A. A., Rankin J. M., 2001, MNRAS, 322, 438 
Detweiler S., 1979, ApJ, 234, 1100

Dib R., Kaspi V. M., Gavriil F. P., 2008a, in C. Bassa, Z. Wang, A. Cumming, \& V. M. Kaspi ed., 40 Years of Pulsars: Millisecond Pulsars, Magnetars and More Vol. 983 of American Institute of Physics Conference Series, 10 Years of RXTE Monitoring of Five Anomalous X-Ray Pulsars. pp 262-264

Dib R., Kaspi V. M., Gavriil F. P., 2008b, ApJ, 673, 1044

Dolch T., Lam M. T., Cordes J., Chatterjee S., Bassa C., Bhattacharyya B., Champion D. J., Cognard I., Crowter K., Demorest P. B., Hessels J. W. T., Janssen G., Jenet F. A., Jones G., Jordan C., Karuppusamy R., Keith M., 2014, ApJ, 794, 21

Duncan R. C., Thompson C., 1994, in Fishman G. J., Brainerd J. J., Hurley K., eds, Gamma-Ray Bursts Astrophysics of very strongly magnetized neutron stars: A model for the soft gamma repeaters. AIP, New York, pp 625-629

Foster R. S., Cordes J. M., 1990, ApJ, 364, 123

Ginzburg V. L., Zheleznyakov V. V., 1970, Comm. Astrophys., 2, 197

Gold T., 1968, Nature, 218, 731

Goldreich P., Julian W. H., 1969, ApJ, 157, 869

Goodman J., Narayan R., 1989, MNRAS, 238, 995

Greidanus H., Strom R. G., 1990, A\&A, 240, 376

Harrison E. R., Tademaru E., 1975, ApJ, 201, 447 
Heiles C., Perillat P., Nolan M., Lorimer D. R., Rhat R., Ghosh T., Lewis B. M., O’Neil K., Salter C., Stanimirovic S., 2001, PASP, 113, 1274

Hellings R. W., Downs G. S., 1983, ApJL, 265, L39

Hemberger D. A., Stinebring D. R., 2008, ApJL, 674, L37

Hessels J. W. T., Ransom S. M., Kaspi V. M., Roberts M. S. E., Champion D. J., Stappers B. W., 2008, in Bassa C., Wang Z., Cumming A., Kaspi V. M., eds, 40 Years of Pulsars: Millisecond Pulsars, Magnetars and More Vol. 983 of American Institute of Physics Conference Series, The GBT350 Survey of the Northern Galactic Plane for Radio Pulsars and Transients. pp 613-615

Hewish A., Bell S. J., Pilkington J. D. H., Scott P. F., Collins R. A., 1968, Nature, 217,709

Hobbs G. B., Edwards R. T., Manchester R. N., 2006, MNRAS, 369, 655

Hotan A. W., van Straten W., Manchester R. N., 2004, Proc. Astron. Soc. Aust., 21,302

Hulse R. A., Taylor J. H., 1975, ApJ, 195, L51

Jackson J. D., 1962, Classical Electrodynamics. Wiley

Jenet F. A., Anderson S. B., Kaspi V. M., Prince T. A., Unwin S. C., 1998, ApJ, 498, 365

Jenet F. A., Anderson S. B., Prince T. A., 2001, ApJ, 546, 394 
Jenet F. A., Hobbs G. B., Lee K. J., Manchester R. N., 2005, ApJL, 625, L123

Jones P. B., 1990, MNRAS, 243, 257

Keane E., Ludovici D. A., Kramer M., 2009, MNRAS

Keane E. F., Kramer M., 2008, MNRAS, 391, 2009

Keane E. F., Kramer M., Lyne A. G., Stappers B. W., McLaughlin M. A., 2011, MNRAS, 415, 3065

Keane E. F., Ludovici D. A., Eatough R. P., Kramer M., Lyne A. G., McLaughlin M. A., Stappers B. W., 2010, MNRAS, 401, 1057

Keith M. J., Coles W., Shannon R. M., Hobbs G. B., Manchester R. N., Bailes M., Bhat N. D. R., Yardley D. R. B., 2013, MNRAS, 429, 2161

Keith M. J., Johnston S., Ray P. S., Ferrara E. C., Saz Parkinson P. M., Çelik Ö., Belfiore A., Donato D., Cheung C. C., Abdo A. A., Camilo F., Freire P. C. C., Guillemot L., Harding A. K., Kramer M., Michelson P. F., Ransom S. M., Romani R. W., 2011, MNRAS, 414, 1292

Kinkhabwala A., Thorsett S. E., 2000, ApJ, 535, 365

Knight H. S., Bailes M., Manchester R. N., Ord S. M., Jacoby B. A., 2006, ApJ, 640,941

Kramer M., Lyne A. G., O’Brien J. T., Jordan C. A., Lorimer D. R., 2006, Science, 312,549 
Kramer M., Xilouris K. M., Lorimer D. R., Doroshenko O., Jessner A., Wielebinski R., Wolszczan A., Camilo F., 1998, ApJ, 501, 270

Kuz'min A. D., Izvekova V. A., 1993, MNRAS, 260, 724

Lattimer J. M., Prakash M., 2007, Phys. Rep, 442, 109

Li X.-D., 2006, ApJL, 646, L139

Liu K., Keane E. F., Lee K. J., Kramer M., Cordes J. M., Purver M. B., 2012, MNRAS, 420, 361

Lorimer D. R., Kramer M., 2005, Handbook of Pulsar Astronomy. Cambridge University Press

Luo Q., Melrose D., 2007, MNRAS, 378, 1481

Lyne A., Hobbs G., Kramer M., Stairs I., Stappers B., 2010, Science, 329, 408

Lyne A. G., Manchester R. N., 1988, MNRAS, 234, 477

Lyne A. G., McLaughlin M. A., Keane E. F., Kramer M., Espinoza C. M., Stappers B. W., Palliyaguru N. T., Miller J., 2009, MNRAS, 400, 1439

McDermott P. N., Van Horn H. M., Hansen C. J., 1988, ApJ, 325, 725

McLaughlin M. A., Lyne A. G., Keane E. F., Kramer M., Miller J. J., Lorimer D. R., Manchester R. N., Camilo F., Stairs I. H., 2009, MNRAS, 400, 1431 
Manchester R. N., Hobbs G., Bailes M., Coles W. A., van Straten W., Keith M. J., Shannon R. M., Bhat N. D. R., Brown A., Burke-Spolaor S. G., Yardley D., Yan W. M., You X. P., 2013, PASA, 30, 17

Manchester R. N., Lyne A. G., Robinson C., Bailes M., D’Amico N., 1991, Nature, 352,219

McLaughlin M. A., 2013, Classical and Quantum Gravity, 30, 224008

McLaughlin M. A., Lyne A. G., Lorimer D. R., Kramer M., Faulkner A. J., Manchester R. N., Cordes J. M., Camilo F., Possenti A., Stairs I. H., Hobbs G., D'Amico N., Burgay M., O’Brien J. T., 2006, Nature, 439, 817

Melikidze G., Gil J., 2006, Chinese Journal of Astronomy and Astrophysics Supplement, 6, 020000

Melrose D. B., 1994, in Kirk J., Melrose D., Priest E., eds, Plasma Astrophysics, SAAS-FEE Advanced courses, Lecture notes Springer, Berlin-Heidelberg

Michel F. C., 1991, Theory of Neutron Star Magnetospheres. University of Chicago Press, Chicago

Miller J. J., McLaughlin M. A., Keane E. F., 2010, MNRAS

Mitra D., Gil J., Melikidze G. I., 2009, ApJL, 696, L141

Mitra D., Rankin J. M., 2011, ApJ, 727, 92

Pacini F., 1968, Nature, 219, 145 
Palliyaguru N. T., McLaughlin M. A., Keane E. F., Kramer M., Lyne A. G., Lorimer D. R., Manchester R. N., Camilo F., Stairs I. H., 2011, MNRAS, 417, 1871

Pen U.-L., Macquart J.-P., Deller A. T., Brisken W., 2014, MNRAS, 440, L36

Press W. H., Flannery B. P., Teukolsky S. A., Vetterling W. T., 1986, Numerical Recipes: The Art of Scientific Computing. Cambridge University Press, Cambridge

Radhakrishnan V., Cooke D. J., 1969, ApJL, 3, 225

Ramachandran R., Demorest P., Backer D. C., Cognard I., Lommen A., 2006, ApJ, 645,303

Rankin J. M., 1983a, ApJ, 274, 333

Rankin J. M., 1983b, ApJ, 274, 359

Rankin J. M., 1986, ApJ, 301, 901

Rankin J. M., 1990, ApJ, 352, 247

Rankin J. M., 1993, ApJ, 405, 285

Ray P. S., Ransom S. M., Cheung C. C., Giroletti M., Cognard I., Camilo F., Bhattacharyya B., Roy J., Romani R. W., Ferrara E. C., Guillemot L., Johnston S., Keith M., Kerr M., Kramer M., Pletsch H. J., Saz Parkinson P. M., Wood K. S., 2013, ApJL, 763, L13 
Rea N., Esposito P., Pons J. A., Turolla R., Torres D. F., Israel G. L., Possenti A., Burgay M., et al., 2013, ApJL, 775, L34

Redman S. L., Rankin J. M., 2009, MNRAS, 395, 1529

Reisenegger A., Goldreich P., 1992, ApJ, 395, 240

Rickett B. J., 1969, Nature, 221, 158

Rickett B. J., 1975, ApJ, 197, 185

Rickett B. J., 1990, ARA\&A, 28, 561

Rosen R., McLaughlin M. A., Thompson S. E., 2011, ApJL, 728, L19

Ruderman M., Shaham J., Tavani M., 1989, ApJ, 336, 507

Ruderman M. A., Sutherland P. G., 1975, ApJ, 196, 51

Sesana A., Sartore N., Devecchi B., Possenti A., 2012, MNRAS, 427, 502

Shannon R. M., Cordes J. M., 2012, ApJ, 761, 64

Stephens M. A., 1970, Journal of the Royal Statistical Society, 32, 192

Stinebring D., 2013, Classical and Quantum Gravity, 30, 224006

Stinebring D. R., Cordes J. M., Rankin J. M., Weisberg J. M., Boriakoff V., 1984, Astrophys. J. Suppl. Ser., 55, 247

Taylor J. H., 1992, Royal Society of London Philosophical Transactions Series A, 341, 117 
Turner M. S., 1997, Phys. Rev. D, 55, 435

van Straten W., 2004, ApJS, 152, 129

van Straten W., Demorest P., Oslowski S., 2012, Astronomical Research and Technology, 9, 237

Verbiest J. P. W., Bailes M., Coles W. A., Hobbs G. B., van Straten W., Champion D. J., Jenet F. A., Manchester R. N., Bhat N. D. R., Sarkissian J. M., Yardley D., Burke-Spolaor S., Hotan A. W., You X. P., 2009, MNRAS, 400, 951

Walker M. A., Demorest P. B., van Straten W., 2013, ApJ, 779, 99

Walker M. A., Koopmans L. V. E., Stinebring D. R., van Straten W., 2008, MNRAS, 388,1214

Wang N., Manchester R. N., Johnston S., 2007, MNRAS, 377, 1383

Weltevrede P., Stappers B. W., Rankin J. M., Wright G. A. E., 2006, ApJL, 645, L149

Williamson I. P., 1972, MNRAS, 157, 55

Woods P. M., Kouveliotou C., Gavriil F. P., Kaspi V. M., Roberts M. S. E., Ibrahim A., Markwardt C. B., Swank J. H., Finger M. H., 2005, ApJ, 629, 985

Xilouris K. M., Kramer M., Jessner A., von Hoensbroech A., Lorimer D., Wielebinski R., Wolszczan A., Camilo F., 1998, ApJ, 501, 286

Zhang B., Gil J., Dyks J., 2007, MNRAS, 374, 1103 
Zhuravlev V. I., Popov M. V., Soglasnov V. A., Kondrat'ev V. I., Kovalev Y. Y., Bartel N., Ghigo F., 2013, MNRAS, 430, 2815 